\title{
Atom trapping and spectroscopy in cavity-generated optical potentials
}

\author{
by \\ Yun-Jhih Chen \\ A dissertation submitted in partial fulfillment \\ of the requirements for the degree of \\ Doctor of Philosophy \\ (Physics) \\ in The University of Michigan \\ 2015
}

Doctoral Committee:

Professor Georg A. Raithel, Chair

Professor Paul R. Berman

Assistant Professor Hui Deng

Professor Theodore B. Norris

Associate Professor Jennifer Ogilvie 


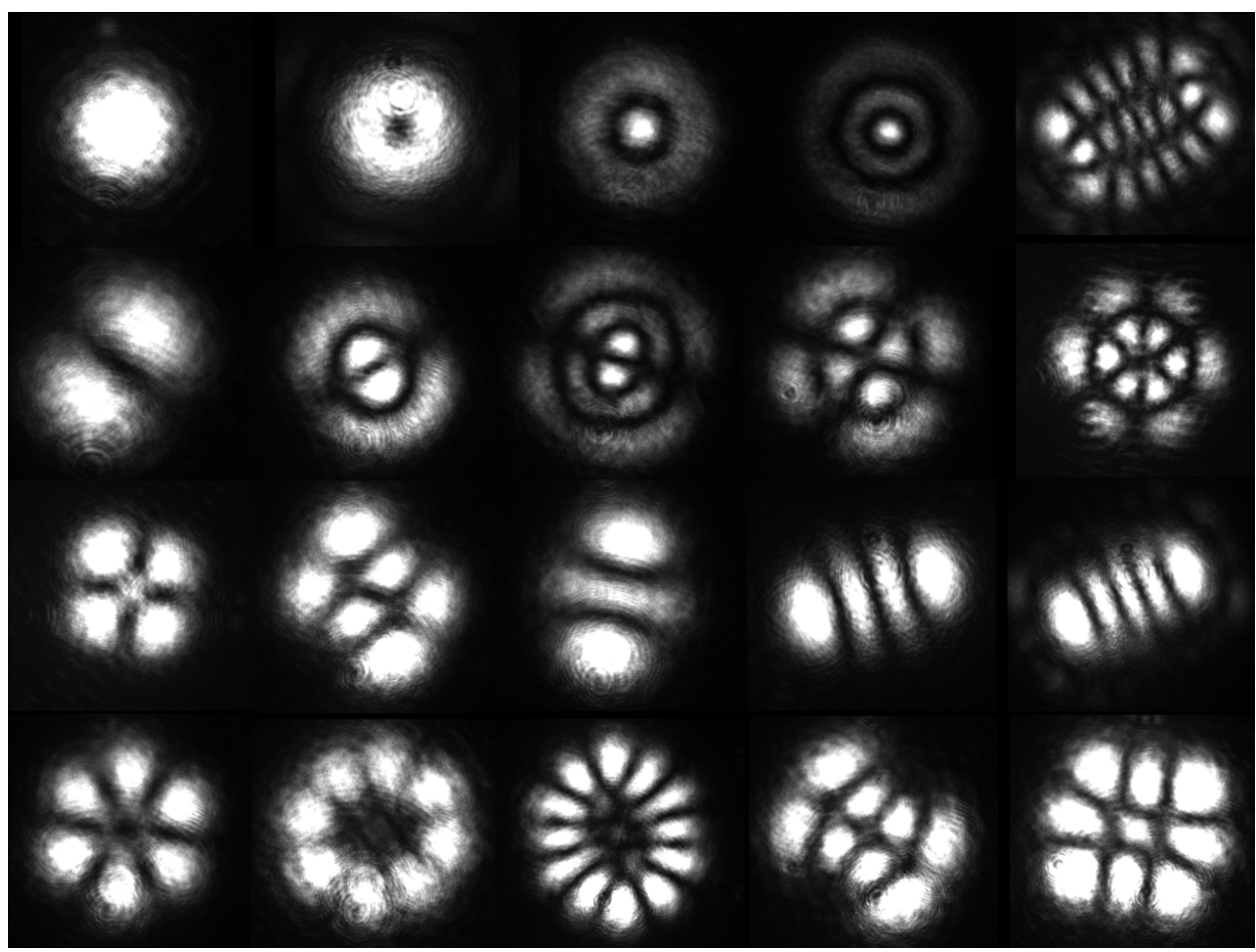


(c) Yun-Jhih Chen 2015

All Rights Reserved 


\section{ACKNOWLEDGEMENTS}

First, I sincerely appreciate my advisor, Georg Raithel. I would not be able to get any of the work presented in this thesis done without him.

I would like to thank all of the people of the Raithel group, for creating a nice working environment and sharing lab tricks. Especially thanks to Stefan Zigo, who is the very first student in this concentric-cavity project, and did the most of early testing and designing of the experiment. Thanks to David Anderson, Luís F. Gonçalves, Jamie MacLennan, Kaitlin Moore and Andira Ramos, for proofreading this thesis and their helpful feedback. Also thanks to Sarah Anderson, for her being my senior graduate student mentor.

I would also like to thank Julian Broad, who had been the supervisor of the student machine shop, for advising me making miscellaneous parts used in the experiment.

I would also like to thank my committee members, including Paul Berman, Hui Deng, Jennifer Ogilvie, and Theodore Norris, for their help to complete this thesis. 


\section{TABLE OF CONTENTS}

ACKNOWLEDGEMENTS . . . . . . . . . . . . . . . . ii

LIST OF FIGURES . . . . . . . . . . . . . . . . . . . . . . vi vi

LIST OF TABLES . . . . . . . . . . . . . . . . . . . . . . . . xiii

LIST OF ABBREVIATIONS . . . . . . . . . . . . . . . . . xiv

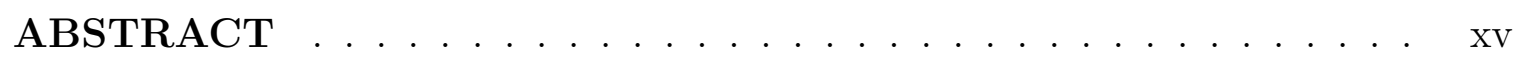

\section{CHAPTER}

I. Introduction $\ldots \ldots \ldots \ldots \ldots \ldots \ldots \ldots$

II. Near-concentric cavity $\ldots \ldots \ldots \ldots \ldots$

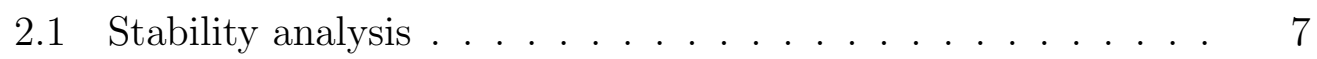

$2.1 .1 \quad$ Cavity modes . . . . . . . . . . . . . . . . . . . 12

2.1 .2 Near-concentric cavity . . . . . . . . . . . . . . . 14

$2.2 \quad$ Optical near-concentric cavity design $\ldots \ldots \ldots \ldots \ldots$

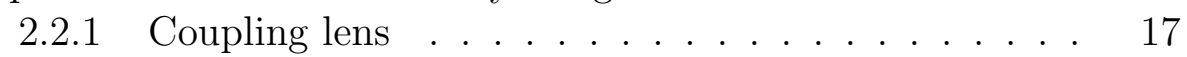

$2.2 .2 \quad$ Cavity mode-matching . . . . . . . . . . . . . . 18

2.2 .3 Theoretical coupling efficiency $\ldots \ldots \ldots \ldots$

2.3 Cavity stabilization with Pound-Drever-Hall scheme . . . . 20

III. Experimental apparatus and techniques $\ldots \ldots \ldots \ldots$

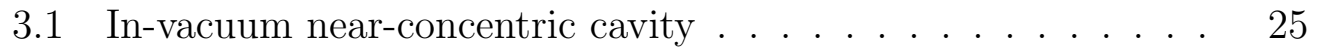

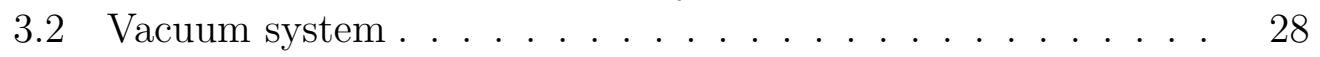

3.3 Excitation lasers $\ldots \ldots \ldots \ldots$

$3.3 .1 \quad$ Amplified DBR laser system . . . . . . . . . . . . 30

3.3 .2 Frequency-doubled blue laser . . . . . . . . . . . . 34

3.3 .3 Pressure-tuned Fabry-Pérot interferometer . . . . . 35 
3.3 .4 Computer control of the experiment . . . . . . . . . 38

3.4 Saturated absorption spectroscopy . . . . . . . . . . . 38

3.5 Magneto-optical trap. . . . . . . . . . . . . . . . . . . . . . . . . . . . . . . . . . 40

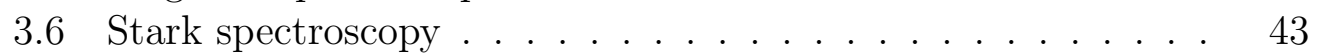

3.7 Optical dipole traps and optical lattices . . . . . . . . . . . . 45

IV. Atom trapping with cavity-generated optical potentials . . . 47

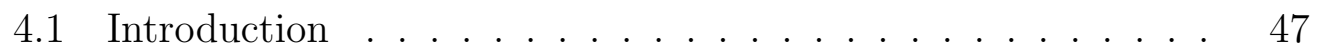

4.2 Fluorescence imaging . . . . . . . . . . . . . . . . . . . . . 49

4.3 Shadow imaging . . . . . . . . . . . . . . . . . . . . . . . . 52

$4.4 \quad$ Spectroscopy and lattice laser cooling limit . . . . . . . . . . 56

$4.5 \quad$ Ionization of ground-state rubidium atoms . . . . . . . . . . . 60

V. Lattice adiabatic compression . . . . . . . . . . . . . . . 64

5.1 Advanced cavity stabilization . . . . . . . . . . . . . 64

5.1 .1 Real-time normalization circuit . . . . . . . . . . 67

5.2 Atom density estimation. . . . . . . . . . . . . . . 70

$5.3 \quad$ Lattice adiabatic compression . . . . . . . . . . . . . . . . 74

5.3 .1 Trap frequency of a deep optical lattice . . . . . . . 75

$5.3 .2 \quad$ Lattice intensity modulation . . . . . . . . . . . . 76

5.3.3 $\quad$ Two-photon excitation spectroscopy . . . . . . . . . 77

VI. Radiation guiding . . . . . . . . . . . . . . . . . . . . . 80

6.1 Imaging system . . . . . . . . . . . . . . . . . . . . . 80

$6.1 .1 \quad$ Radiation guiding as an experimental alignment tool in Rydberg atom spectroscopy . . . . . . . . . . 82

6.2 Radiation guiding . . . . . . . . . . . . . . . . 83

6.2 .1 Timing sequence . . . . . . . . . . . . . . . . . 83

6.2 .2 Lensing effect . . . . . . . . . . . . . . . 85

6.3 Qualitative model ................ . . 88

VII. Dynamic polarizability measurement . . . . . . . . . . . . . . 92

$7.1 \quad$ Introduction - Stark shift and dynamic polarizability . . . . . 92

7.2 Two-photon excitation in deep optical lattices: numerical sim-

ulation . . . . . . . . . . . . . . . . . . . 98

7.2 .1 "Magic" Rydberg state . . . . . . . . . . . . 98

7.2 .2 Two-photon excitation resonances . . . . . . . . . 100

7.2 .3 Spectral line broadening . . . . . . . . . . . 102

7.3 Polarizability measurement . . . . . . . . . . . . . . . 105

7.3 .1 Methods . . . . . . . . . . . . . . 105

7.3 .2 Spectral line shape . . . . . . . . . . . 107 
$7.3 .3 \quad$ Experimental values of $\alpha_{0}$ and $\alpha_{2} \quad \ldots . . . \quad . \quad . \quad 109$

VIII. Spectroscopy of hydrogenic Rydberg states . . . . . . . . . . . 112

8.1 Ponderomotive optical lattices . . . . . . . . . . . . . . . . 112

8.2 Experimental progress . . . . . . . . . . . . . . . . . 116

IX. Summary and future works . . . . . . . . . . . . . . . . . . . . . 120

APPENDIX . . . . . . . . . . . . . . . . . . . . . . . . . 122

BIBLIOGRAPHY . . . . . . . . . . . . . . . . . . . . . 125 


\section{LIST OF FIGURES}

\section{$\underline{\text { Figure }}$}

\begin{tabular}{|c|c|}
\hline 2.1 & Gaussian beam inside a two-mirror optical cavity. The propagating \\
\hline & direction is along z-axis. Red curves: mirror surfaces of mirror $1\left(\mathrm{M}_{1}\right)$ \\
\hline & and mirror $2\left(\mathrm{M}_{2}\right) . \quad \ldots \ldots \ldots \ldots \ldots$ \\
\hline 2.2 & Stability diagram of two-mirror cavities. Origin: wikipedia (http:// \\
\hline & commons.wikimedia.org/wiki/File:Laser_resonator_stability. \\
\hline & svg) $\ldots \ldots \ldots \ldots \ldots \ldots \ldots$ \\
\hline 2.3 & (a) Near-concentric cavity. A pair of coupling lenses is required to \\
\hline & \begin{tabular}{|l|l|l} 
focus a collimated input beam at the cavity center. See Sec. & 2.2 & for \\
\end{tabular} \\
\hline & more information. (b) Cavity transmission spectra illustration and \\
\hline & the mostly seen four Hermite-Gaussian (HG) cavity modes. . . . . . \\
\hline 2.4 & Illustration of transmission spectra for near-planar, confocal, and \\
\hline & near-concentric cavities with the same cavity length. near-planar: \\
\hline & $g_{1}, g_{2} \rightarrow 1^{-}, \frac{\cos ^{-1} \sqrt{g_{1} g_{2}}}{\pi} \rightarrow 0^{+}$. confocal: $g_{1}, g_{2}=0, \frac{\cos ^{-1} \sqrt{g_{1} g_{2}}}{\pi}=\frac{1}{2}$ \\
\hline & near-concentric: $g_{1}, g_{2} \rightarrow-1^{+}, \frac{\cos ^{-1} \sqrt{g_{1} g_{2}}}{\pi} \rightarrow 1^{-}$. The cavity modes \\
\hline & for both near-planar and near-concentric cavities are non-degenerate, \\
\hline & " while the cavity modes of a confocal cavity are degenerate. . . . . . \\
\hline 2.5 & Focusing collimated input beam into a thick cavity mirror with a thin \\
\hline & coupling lens. $w_{1}$ is the waist of the Gaussian cavity mode $\left(\mathrm{HG}_{00}\right)$ \\
\hline & \begin{tabular}{|l|l} 
on the cavity mirror surface. $w_{1}^{\prime}$ is the waist on the thin focusing lens. & 17
\end{tabular} \\
\hline 2.6 & Profiles of the laser beam rejected by the cavity when the cavity is \\
\hline & locked to Hermite-Gaussian (HG) $00,10,20$, and 30 modes. The \\
\hline & input beam is Gaussian. The cavity extracts the portion of input \\
\hline & which matches the cavity modes and then rejects the rest, leaving \\
\hline & dark strips on the profile of the return beam. . . . . . . . . . . \\
\hline 2.7 & A oscilloscope screenshot of the cavity reflection (yellow) and PDH \\
\hline & $\begin{array}{l}\text { error signal (blue). } \\
\end{array}$ \\
\hline 2.8 & Layout of the Pound-Drever-Hall cavity stabilization with commercial \\
\hline & control modules . . . . . . . . . . . . . . . . . . . . . . . . . . . . . \\
\hline 3.1 & (a) Experimental geometry. The cavity is stabilized to the $1064 \mathrm{~nm}$ \\
\hline & lattice laser. A Rb MOT is produced directly at the cavity center, \\
\hline & loading the optical lattice trap at the focus where the intensity is the \\
\hline & highest. (b) Photo of the concentric-cavit \\
\hline
\end{tabular}


3.2 Left: concentric tubes viewed from the coupling lens side. The Kapton wires connect to a ring piezo placed in-between the tubes. Middle: concentric tubes viewed from the side of top cavity mirror. Right: six electrodes inside the stainless steel cylinder. Sec. 3.6 describes the usage of the electrodes. . . . . . . . . . . . . . . . . . 27

3.3 Left: CAD drawing of the tripod and photo of a conventional mirrormount. Right: hand-made adaptor for the three-point contact scheme. Close look of the tripod. The red/white wire pairs connect to the plate piezos. . . . . . . . . . . . . . . . . 28

3.4 Cavity inside the vacuum chamber seen from the large viewport on \begin{aligned} \hline the top of the vacuum chamber. The aluminum cone next to the cav- \\ \hline\end{aligned} ity is an ion guard tube, which shields the field-ionized ions on their way to the microchannel plate (MCP) in spectroscopy experiments.

3.5 Vacuum chamber front view. The neodymium magnets provide the quadruple magnetic field required for the MOT. The three pairs of offset coils fine tune the MOT magnetic field. The neodymium magnets are 3/4-inch in diameter and 2 inches long. They are wrapped with a piece of paper and fixed inside a 1-inch plastic tube adaptor by frictional force. . . . . . . . . . . . . . . . . . . . . 30

$3.6 \quad$ Amplified DBR laser system. The components are all mounted on fixed-height, customized pedestals. Some of them can freely slide on the bottom aluminum rails. . . . . . . . . . . . . . . . . 32

\begin{tabular}{|l|l|l|}
\hline fixed-height, customized pedestals. Some of them can freely slide on \\
\hline \hline the bottom aluminum rails. . . . . . . . . . . . . . . . . 32 \\
\hline
\end{tabular}

$3.7 \quad$ (a) Fabry-Pérot interferometer transmission spectrum (top) and saturated absorption spectrum for rubidium-85 (bottom). The DBR laser diode is current modulated, and its output is sampled for both spectra at the same time. (b) A zoom-in of the saturated absorption spectra. The absorption peaks are fitted with Lorentz amplitude function. The validity of Lorentz peak-findings is examined by comparing the centers of the crossover peaks[1] to their expected position, which is in the middle of two real peaks. The difference is within $0.1 \%$. The centers of the $\mathrm{F}=3 \rightarrow 4$ and $\mathrm{F}=2 \rightarrow 3$ transition gives the frequency reference $2915.092439 \mathrm{MHz}$. . . . . . . . . . . . 33

3.8 Left: center pieces of the TA amplifier laser. Right: TA amplifier laser assembly. ....................... 35

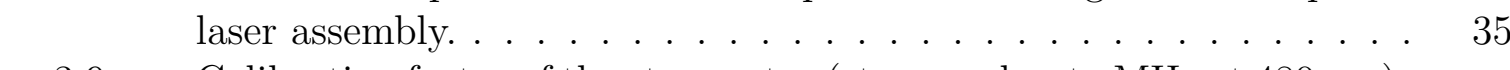

3.9 Calibration factor of the step motor (step number to $\mathrm{MHz}$ at $480 \mathrm{~nm}$ ). The slight change in calibration is mostly due to the non-linear response of the bellow. . . . . . . . . . . . . . . . . . 36

$3.10960 \mathrm{~nm}$ FPI transmission spectra. The side bands are caused by higher-order deflections of a single-pass AOM. Note the response of the grating modulation is not as linear as the current response of a DBR laser. The ratio of the FSR to sidedands is used in the calculation to compensate this nonlinearality. . . . . . . . . . . . . . 37

3.11 Wide scan of absorption spectra of rubidium $5 \mathrm{~S}_{1 / 2} \rightarrow 5 \mathrm{P}_{3 / 2}$ transition. 39

$3.12 \quad$ Saturated absorption spectra for rubidium 85 and $87 . \quad$. . . . . . 41 
3.13 Stark maps for the rubidium 67D level. The electric field in $\mathrm{x}$ direction is controlled by four of the electrodes surrounding the cavity. The four electrodes are marked by solid disks. The electric field in y direction is controlled by the other two electrodes, marked by open circles. The stray electric field is compensated at the voltage setting where the lines have no splitting. The maximal upper-shift in frequency of $67 \mathrm{D}_{5 / 2}$ lines happens at $-0.21 \mathrm{~V}$ and $1.12 \mathrm{~V}$ of $\mathrm{x}$-axis scan, and $-0.70 \mathrm{~V}$ and $0.62 \mathrm{~V}$ of $\mathrm{y}$-axis scan. These maxima on the map give a rough calibration of electric field. . . . . . . . . . . . . . 44

$3.14 \quad$ two-photon excitation, shallow lattice spectrum. . . . . . . . . . . . 46

$4.1 \quad$ Cavity-generated optical lattice potentials are nearly perfect. Dotted lines are unshifted energy levels, while the solid lines are latticeshifted levels. The gap between $5 \mathrm{~S}$ and $5 \mathrm{P}$ is not to scale. . . . . . 48

4.2 Top camera screen shots of the bright optical lattice traps inside the thin cloud of a MOT. The cavity is locked to $\mathrm{HG}_{00}, \mathrm{HG}_{10}, \mathrm{HG}_{20}, \mathrm{HG}_{30}$

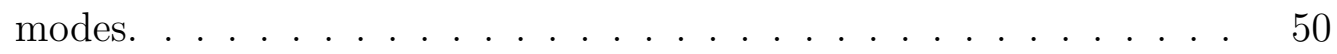

4.3 Top: $780 \mathrm{~nm}$ fluorescence of the MOT overlapped with $\mathrm{HG}_{00}$ modes with the indicated transmitted laser powers. The exposure time of each image is $50 \mathrm{~ms}$. Bottom: Horizontal cuts of the images averaged over 3 to 5 rows of pixels. The box on the first image shows the spatial range of the cuts. . . . . . . . . . . . . . . 51

\begin{tabular}{|l|l|}
\hline with the indicated transmitted laser powers. The exposure time of \\
\hline \hline each image is 50 ms. Bottom: Horizontal cuts of the images averaged \\
\hline \hline over 3 to 5 rows of pixels. The box on the first image shows the spatial \\
\hline \hline range of the cuts. . . . . . . . . . . . . . . . . . . . . . . . & 51 \\
\hline
\end{tabular}

4.42 imaging: because the photon-scattering of probe light by the atom cloud is radially outward in all directions, the shadow of atom cloud images sharply on the image plane, while the probe light does not. $\mathrm{f}_{1}=15 \mathrm{~cm}, \mathrm{f}_{2}=30 \mathrm{~cm}$, the distance between the lenses is irrelevant. This lens configuration gives magnification factor of $2.2 . . . .53$

4.5 Middle: absorption images of atoms inside the lattice fields, in the plane transverse to the lattice axis. Transmitted lattice laser powers, from left to right, are 1, 1.6, 1.9 and $1.6 \mathrm{~mW}$. The probe detuning is $27 \mathrm{MHz}$ for modes $\mathrm{HG}_{00}, \mathrm{HG}_{10}$, and $\mathrm{HG}_{20}$, and $25 \mathrm{MHz}$ for mode $\mathrm{HG}_{30}$. Top: corresponding calculated area densities of on-resonant atoms. Bottom: calculated electric-field squares of $\mathrm{HG}_{00}, \mathrm{HG}_{10}$, and $\mathrm{HG}_{20}$ and $\mathrm{HG}_{30}$, for a circulating laser power of $1 \mathrm{~W}$ and $w_{0}$ of $23 \mu \mathrm{m}$. 54

4.6 Absorption images of atoms inside the HG lattices and corresponding area density calculation in the $x z$-plane. The aspect ratio of each cold-atom cloud is about 10 to 15. The transmitted lattice laser powers and probe detunings are the same as in Fig. 4.5. Atom numbers computed using the on-resonant scattering cross section are 5400, 4200, 7500 and 6000, respectively; the numbers are of on-resonant atoms. . . . . . . . . . . . . . 


\begin{tabular}{|c|c|}
\hline \multirow[t]{7}{*}{4.7} & Two-photon excitation spectra of Rb 60D levels. Spectral resolution \\
\hline & is $\approx 5 \mathrm{MHz}$. (a) Spectra with transmitted lattice laser power $1.2 \mathrm{~mW}$, \\
\hline & and the indicated lower-transition detunings $\Delta$. (b) Spectra for the \\
\hline & indicated transmitted lattice laser powers and $\Delta=75 \mathrm{MHz}$. The \\
\hline & \multirow{2}{*}{ experimental repetition rate is $100 \mathrm{~Hz}$. Each data point is an average } \\
\hline & \\
\hline & light. . . . . . . . . . . . . . . . . . . . \\
\hline 4.8 & 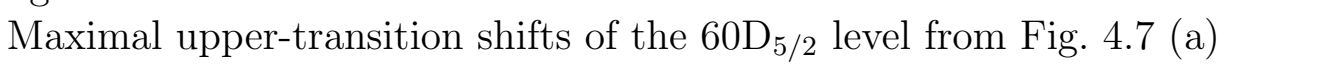 \\
\hline & vs lower-transition detuning $\Delta$ and (b) vs transmitted laser power. \\
\hline & The amount of shift is estimated by the frequency distance between \\
\hline & the central position of the narrow line to the broadened blue-shifted \\
\hline & feature, plus half of the $1 / e^{2}$ full width of Gaussian fits to the blue- \\
\hline & shifted feature. In the cases that the blue-shifted feature shows mul- \\
\hline & tiple peaks, the one at larger detuning is used. The dotted line in (a) \\
\hline & indicates that the lattice induced shift converges to an asymptotic \\
\hline & value of about $30 \mathrm{MHz}$. The straight line in (b) shows that at low \\
\hline & powers the calibration for the ground-state atom trap depth is about \\
\hline & 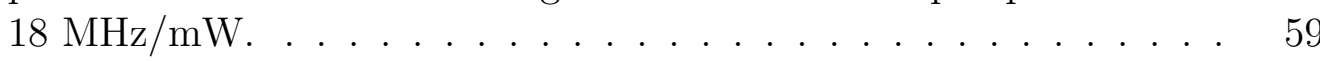 \\
\hline 4.9 & Average of the blips on MCP phosphor screen when cavity is locked \\
\hline & to $\mathrm{HG}_{00}$ mode and $\mathrm{HG}_{10}$ modes. The blips reflect the spatial dis- \\
\hline & tribution of the cavity modes. $\mathrm{HG}_{00}$ : average of 800 shots, $\mathrm{HG}_{10}$ : \\
\hline & average of 1000 shots. The image of the $\mathrm{HG}_{10}$ mode shows the ions \\
\hline & originate from two sources whose spacing is about or less than $20 \mu \mathrm{m}$ \\
\hline & (i.e. the lobe spacing of the $\mathrm{HG}_{10}$ cavity mode, see Figure Fig. 4.5$)$. \\
\hline & \\
\hline & rate drops when either MOT light (including cycling and repumping \\
\hline & transitions) or lattice laser is turned off. (b) Count rate returns to \\
\hline & high level when the $10 \mu$ s probe (cycling transition $5 \mathrm{~S}_{1 / 2} \rightarrow 5 \mathrm{P}_{3 / 2}$ ) \\
\hline & pulses on. $\ldots \ldots \ldots \ldots \ldots \ldots$ \\
\hline & Optics layout for the Pound-Drever-Hall stabilization scheme with \\
\hline & lattice amplitude modulation. See text for details. EOM: electro- \\
\hline & optic modulator. AOM: acousto-optic modulator. . . . . . . . . . . \\
\hline 5.2 & A screenshot of cavity transmitted power (yellow) monitor by a pho- \\
\hline & todiode. Blue curve is a multiplexer pulse. . . . . . . . . . . . . . . \\
\hline & (a) logarithmic output. (b) exponential output. \\
\hline & \\
\hline
\end{tabular}




\begin{tabular}{|c|c|}
\hline \multirow[t]{17}{*}{5.5} & Central volume density $n_{V}(0,0,0)=n_{A}(0,0) \frac{1}{\sigma \sqrt{2 \pi}}$ estimate by ab- \\
\hline & sorption imaging. MOT atoms are first loaded into a shallow lattice \\
\hline & with a power of $2 \mathrm{~mW}$. The MOT laser is turned off at the end of \\
\hline & the loading, and the lattice starts compressing the atoms to the bot- \\
\hline & tom of lattice trap as the lattice power increases to $40 \mathrm{~mW}$ in $30 \mu \mathrm{s}$. \\
\hline & After the twentyfold compression, the lattice laser holds at $40 \mathrm{~mW}$ \\
\hline & for $70 \mu \mathrm{s}$, and then is switched off. A $5 \mu \mathrm{s}$ probe beam, which is \\
\hline & tuned to the MOT cycling transition, shines through the entire sam- \\
\hline & ple immediately after the lattice is off. Left: area density plot of the \\
\hline & lattice trap inside the MOT. The thin strips immediately to the left \\
\hline & of the trap are caused by probe light diffraction, suggesting a very \\
\hline & high atom density inside the lattice trap. Upper right: area density \\
\hline & \\
\hline & integrated density. The FWHM of the lattice trap is $16.5 \mu \mathrm{m}$. The \\
\hline & Gaussian fit also gives $\sigma=7.03 \mu \mathrm{m}$, so $\frac{1}{\sigma \sqrt{2 \pi}}=567 \mathrm{~cm}^{-1}$. From the \\
\hline & areas density plot, the area density $n_{A}(0,0)$ is above $1 \times 10^{9} \mathrm{~cm}^{-2}$. \\
\hline & \begin{tabular}{l|l} 
The central volume density is then estimated to be above $5 \times 10^{11} \mathrm{~cm}^{-3}$. & 72
\end{tabular} \\
\hline 5.6 & FWHM of the atom density distribution in lattices of (a) different \\
\hline & compression ratio (b) different sine-rising duration. The oscillation \\
\hline & of FWHM after $100 \mu \mathrm{s}$ might be due to the heating of the trapped \\
\hline & atoms by the intense lattice laser field. $\ldots \ldots \ldots \ldots$ \\
\hline 5.7 & Two-photon excitation spectra. (a) Lattice is always at loading \\
\hline & power. (b) Lattice is at loading power for most of the time, but \\
\hline & compressed to high power immediately before the Rydberg excita- \\
\hline & tion. The splitting of $74 \mathrm{~S}$ signal is due to the intermediate state \\
\hline & 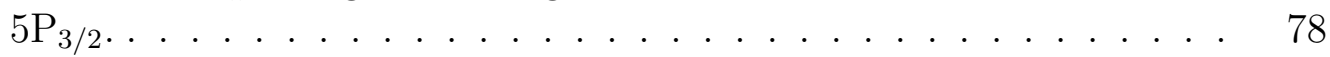 \\
\hline 6.1 & Illustration of the laser light path in the shadow/fluorescence imag- \\
\hline & ing. Not shown in the plot: a long pass dichroic mirror that separates \\
\hline & the $780 \mathrm{~nm}$ and $1064 \mathrm{~nm}$ laser light before the lowpass filter. . . . . \\
\hline & Lower transition laser lensing by the atoms in a shallow lattice trap. \\
\hline & The lower transition laser is focused in, and its waist $(50 \mu \mathrm{m})$ is \\
\hline & about twice of the waist of the cavity mode $(23 \mu \mathrm{m})$. The number is \\
\hline & the order of the screenshots, as the lower transition frequency sweep \\
\hline & through the cycling transition frequency. $\ldots$. . . . . . . . . . . \\
\hline 6.3 & Illustration of the timing sequence. Orange: lattice laser power. Blue: \\
\hline & probe pulse. $t_{1}:$ compression duration. $t_{2}:$ hold high. $t_{3}:$ probe pulse \\
\hline & delay. . . . . . . . . . . . . . . . . . . . . \\
\hline 6.4 & Shadow images of a lattice compressed atom sample at various probe \\
\hline & pulse delays. The bright background is the probe light field, which is \\
\hline & banded because of interference effects caused by the vacuum window \\
\hline & or other optics components along the beam path. The dark disk at \\
\hline & the center is the shadow of the lattice-compressed atom sample, and \\
\hline & it is usually completely saturated. 1 pixel $=2.45 \mu \mathrm{m}$ in the object \\
\hline & plane. \\
\hline
\end{tabular}


6.5 Radiation guiding in a lattice-compressed ${ }^{87} \mathrm{Rb}$ sample. The uncer\begin{tabular}{|l}
\hline tainty of the probe frequency is $\pm 1 \mathrm{MHz} .1$ pixel $=2.45 \mu \mathrm{m}$ in the \\
\hline \hline object plane. . . . . . . . . . . . . . . . . 87
\end{tabular}

6.6 Dispersion curve, using Eq. 6.2 . . . . . . . . . . . . . . . . . . . . 88

6.7 Index of refraction and the absorption in the transverse direction, using Eq. 6.2] . . . . . . . . . . . . . . . . . . . . 89

6.8 Probe light wavefront phase and amplitude after passing the trap.

\begin{tabular}{|c|}
\hline (a) red-detuned probe. (b) blue-detuned probe. The dotted line in \\
\hline \hline both plot
\end{tabular}
both plot is the amplitude of the on-resonance probe. . . . . . . . . 91

$7.1 \quad$ The a.c. Stark light shift of the transitions $5 \mathrm{~S}_{1 / 2} \rightarrow 5 \mathrm{P}_{3 / 2}$ of ${ }^{87} \mathrm{Rb}$ $\left({ }^{85} \mathrm{Rb}\right)$ relative to the on-resonance cycling transition $5 \mathrm{~S}_{1 / 2}, F=$ $2(3) \rightarrow 5 \mathrm{P}_{3 / 2}, F^{\prime}=3(4)$. The plotted energy level shift is a combination of the light shifts of these two levels. The ground-state $5 \mathrm{~S}_{1 / 2}$ sublevels have the same a.c. shift, and the splitting here are caused by the upper level $5 \mathrm{P}_{3 / 2}$. The calculation uses $\alpha_{5 \mathrm{~S}}\left(5 \mathrm{~S}_{1 / 2}\right)=750 \times 4 \pi \epsilon_{0} \mathrm{a}_{0}^{3}$, $\alpha_{0}\left(5 \mathrm{P}_{3 / 2}\right)=-800 \times 4 \pi \epsilon_{0} \mathrm{a}_{0}^{3}$, and $\alpha_{2}\left(5 \mathrm{P}_{3 / 2}\right)=500 \times 4 \pi \epsilon_{0} \mathrm{a}_{0}^{3}$ as a trial number in the Hamiltonian. Although later-on with the experimental data input, we know these number are not accurate, but qualitatively the level splitting and crossing behavior is correct. . . . . . . . . . . 95

7.2 Relevant levels of a two-photon Rydberg excitation. Dotted lines are unshifted energy levels, while the solid lines are lattice-shifted levels. The gaps between levels are not to scale. (a) No light-shift, onresonance two-photon excitation. (b)(c) With light shift in a $1064 \mathrm{~nm}$ lattice. The $74 \mathrm{~S}$ state has a constant light shift along z-axis. . . . . 98 Illustration of the resonance-seeking. (a) The open circles mark the resonances. When the lower-transition detuning relative to the cycling transition is $\Delta$ red, the energy levels are on resonance with the lower transition at different lattice intensities. (b) Top: sketch of a two-photon excitation spectrum with fixed lower transition detuning $\Delta$ red. Bottom: energy levels. Transition frequencies: $\mathrm{R}_{1}$ is the on-resonance cycling transition $\left(5 \mathrm{~S}_{1 / 2} \rightarrow 5 \mathrm{P}_{3 / 2}\right)$, and $\mathrm{B}_{1}$ is the on-resonance upper transition frequency. $\mathrm{R}_{2}=\mathrm{R}_{3}=\mathrm{R}_{1}+\Delta$ red $=$ $\mathrm{R}_{1}+\Delta 5 \mathrm{~S}+\Delta 5 \mathrm{P} . \Delta$ blue $=\mathrm{B}_{3}-\mathrm{B}_{2}=\Delta 74 \mathrm{~S}+\Delta 5 \mathrm{~S}$. . . . . . . . . 101

$7.4 \quad$ Calculated two-photon excitation spectra for the $74 \mathrm{~S}$ magic state of $\begin{array}{lll}{ }^{87} \mathrm{Rb} \text { in a } 1064 \mathrm{~nm} \text { lattice. The plot shows discrete resonances spots. } & 102\end{array}$

\begin{tabular}{|c|c|}
\hline 7.5 & $\begin{array}{l}{ }^{87} \mathrm{Rb} \text { in a } 1064 \mathrm{~nm} \text { lattice. The plot shows discrete resonances spots. } \\
\text { Calculated two-photon excitation spectra for } 74 \mathrm{~S} \text { magic state in } 1064 \mathrm{~nm}\end{array}$ \\
\hline & lattice. The plot includes the effects of off-resonant excitation. The \\
\hline & slope of the spectral lines are insensitive to the lattice power, as seen \\
\hline & in the plot. Note that we have overlaid the results obtained for three \\
\hline & different peak lattice intensities in the same plot). . . . . . . . . . . \\
\hline 7.6 & Experimental spectra overlaid over a simulated contour plot. Exper- \\
\hline & iment: lattice power $20 \mathrm{~mW}$, excitation pulse duration $2 \mu \mathrm{s}$. Simula- \\
\hline & tion: lattice intensity $1.8 \times 10^{11} \mathrm{~W} / \mathrm{cm}^{2} \quad \ldots \ldots$ \\
\hline
\end{tabular}


7.7 (a) "Ellipses" of equal lattice laser intensity. Intensity: $I_{1}>I_{2}$, eccentricity: $\epsilon_{2}>\epsilon_{1}$. (b) Light shift of the magic Rydberg state. (c) triangular line shape: $\delta$ is due to the laser linewidth, and L is due to the lattice intensity variation in space. . . . . . . . . . . . . . 108

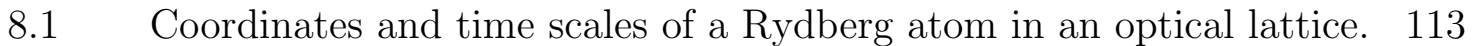

8.2 Adiabatic levels with full free-electron POL depth of $1 \mathrm{GHz}$ (a) hydrogenic manifold vs center-of-mass position $\mathrm{z}$ in a one-dimensional POL $(\lambda=1064 \mathrm{~nm})$. $\mathrm{nH}=$ all the high-angular momentum state ("hydrogen-like" state) at principal quantum number $n$. The states in the plot follow this order (top-down): $n H,(n+3) S,(n+1) D,(n+2) P$ (b) Zoom-in of hydrogenic states at $n=66$. (c) theoretical excitation rate of $66 \mathrm{H}$ for estimated experimental conditions. . . . . . . . . . . 115

$8.3 \quad$ Relative spectral-line positions near the $66 \mathrm{H}$ in $\mathrm{GHz} . \quad$. . . . . . . . . 117

$8.4 \quad$ Experimental scan that shows possible, but not certain, signal from the lattice-shifted hydrogenic states. . . . . . . . . . . . . . . . . . . 118

A.1 $69 \mathrm{~S}$ spectra for ${ }^{87} \mathrm{Rb}$. The atoms are from the $5 \mathrm{P}_{3 / 2},\left|m_{j}\right|=\frac{1}{2}$ intermediate state. The lower transition laser co-propagates with the lattice laser, and is focused into the cavity with an intensity about

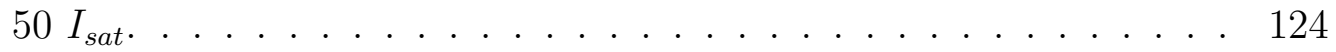




\section{LIST OF TABLES}

\section{$\underline{\text { Table }}$}

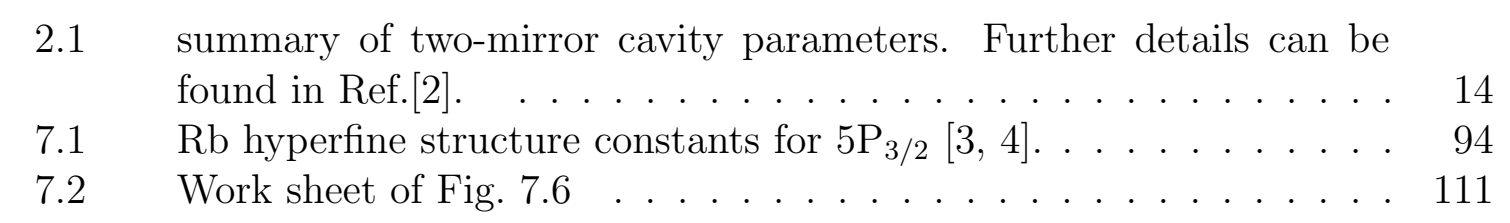




\section{LIST OF ABBREVIATIONS}

AOM acousto-optic modulator

DBR Distributed Bragg Reflector

ECDL external cavity diode laser

EOM electro-optic modulator

FPI Fabry-Pérot interferometer

FSR Free spectral range

FWHM full-width-half-maximum

HG Hermite-Gaussian

MCP microchannel plate

MOT Magneto-optical trap

PDH Pound-Drever-Hall

PID proportional-integral-derivative

POL ponderomotive optical lattice

$\mathbf{R F}$ radio frequency

sat. spec. saturated absorption spectroscopy

TA Tapered Amplifier

TTL Transistor-transistor Logic 


\begin{abstract}
Atom trapping and spectroscopy in cavity-generated optical potentials by Yun-Jhih Chen
\end{abstract}

Chair: Georg Raithel

In this thesis I study atom trapping in GHz-deep optical lattices, generated by an in-vacuum near-concentric optical cavity at $1064 \mathrm{~nm}$, to perform experiments on cold Rydberg atoms. In contemporary atomic physics, cold Rydberg atoms are widely used due to their high sensitivity to static and ac fields, as well as to their unusual collision properties. In my research, I intend to study the response of such atoms to GHz-deep optical traps. In the atom preparation procedure, the deep optical-lattice trap adiabatically compresses the cold rubidium atom sample within the lattice wells, where the atoms experience light shifts of several GHz. The deep optical-lattice trap allows me to perform several spectroscopy experiments which have not yet been done. An experimental challenge that had to be overcome was the realization of GHz-deep light shift traps (which are highly unusual in the field). The design of the cavity experiment also allows a fast experimental repetition rate (which is advantageous in spectroscopy experiments), a large atomic number density, and cold-atom samples with a highly elongated aspect ratio.

A near-concentric cavity is the only type of stable two-mirror cavity that has a 
focus at the cavity center. This configuration not only provides high laser intensity at the cavity center, but also nearly perfect three-dimensional optical trapping potential based on the cavity's non-degenerate cavity modes. The cavity-generated optical trapping potentials offer a platform for deep ponderomotive Rydberg spectroscopy which would be otherwise very difficult in a conventional optical-lattice experimental setup.

In this thesis, I discuss the work that I have achieved for constructing and realizing this unique in-vacuum near-concentric cavity experimental tool from the beginning. I present the recent experimental results with the cavity-generated optical-lattice experiment: I discuss the critical technique I have established to perform lattice adiabatic compression of the cold-atom sample. I have realized atom trapping and spectroscopy in GHz-deep optical lattices. I observe a lensing effect by the lattice-compressed elongated cold-atom cloud prepared in the cavity-generated trapping potential, including the radiation guiding at the trap center, and the surface guiding around the trap surface. I measure the scalar and tensor polarizability of the rubidium $5 \mathrm{P}_{3 / 2}$ level in the intense 1064-nm lattice light field, and I obtain $\alpha_{0}=-1112 \times 4 \pi \epsilon_{0} a_{0}^{3}$ and $\alpha_{2}=535 \times 4 \pi \epsilon_{0} a_{0}^{3}$. This result can be useful to test theoretical models; it is also of general interest in the atom trapping community (where rubidium and $1064 \mathrm{~nm}$ laser traps are commonly used). The GHz-deep cavity-generated optical lattices also offers an opportunity to study the adiabatic energy levels of lattice-mixed hydrogenic states in optical lattices. I summarize my effort to map the lattice-mixed hydrogenic Rydberg state with an initial experimental spectrum. 


\section{CHAPTER I}

\section{Introduction}

Rydberg atoms are atoms in a highly-excited state, in which the outermost electron, called Rydberg electron, is in an energy level with large principal quantum number $n$ (usually $n>30$ ). A Rydberg atom is thousands of times larger than a ground-state atom, and its corresponding atomic radius scales as $2 n^{2}$ at low and $n^{2}$ at high angular momentum. As a result, the Rydberg electron, which is quasi-free at this atomic radius, interacts with the core of the Rydberg atom in a similar way as the electron of a hydrogen atom interacts with its atomic nucleus. The binding energy of the Rydberg electron is approximated by a modified Bohr formula [5],

$$
E_{n l}=-h c \frac{\mathrm{Ryd}}{\left[n-\delta_{l}\right]^{2}}
$$

where Ryd is the Rydberg constant, $n$ is the principal quantum number, and $\delta_{l}$ is the quantum defect, which is subtracted from $n$ to account for the effect of the ionic core potential. $\delta_{l}$ depends on angular momentum $l$. In rubidium, $\delta_{l}=3.13$ for S-states. For $l \geq 3$, the quantum defects are $\ll 1$.

Rydberg atoms have several exaggerated properties. For example, they are longlived (the lifetime scales as $n^{3}$ for low angular momentum) and extremely sensitive to external electric and magnetic fields. These unusual properties make Rydberg atoms an interesting topic in atomic physics. For example, Rydberg atoms can be used to 
measure microwave electric field [6], since the transition energy between the Rydberg states is in the $\mathrm{GHz}$ to $\mathrm{THz}$ range, and transition dipole moments are large (they scale as $n^{2}$ ). Rydberg atoms can also be used to measure Rydberg constant. In a lithium atom beam experiment [7], the Rydberg constant is determined by the measurement of the microwave transition between two consecutive circular Rydberg states (states with $\left|m_{l}\right|=n-1$ ), whose valence electron distribution has the shape of a thin torus.

Rydberg atoms are also promising candidates for the realization of quantum computing [8 10]. This is because the interaction between Rydberg atoms are strong, while the interaction between Rydberg atoms and the environment is weak, assuming sufficiently precise and accurate stray field control as well as low background gas density. This properties enable fast and reliable quantum gate operation. The gate operation of Rydberg atoms is embedded in the usage of two hyperfine ground-state levels, and the control of laser excitation to the Rydberg state. The experimental demonstration of entanglement between Rydberg atoms and the demonstration of a quantum phase gate and a controlled-NOT gate can be found in references [11-13].

The production of Rydberg atoms in laboratory is usually achieved by optical excitation of ground-state atoms. (Interestingly, Rydberg atoms exists naturally in interstellar space, where they have been observed via radio frequency detection.) In this thesis, I utilize two-photon excitation to produce the Rydberg atoms from the ground-state atoms trapped inside a Magneto-optical trap. The Rydberg atoms in my experiment are detected by field ionization with moderately high voltage. This is possible because the Rydberg electron is loosely bound and easy to be ionized. Based on this well-established production and detection scheme, I study the trapping of Rydberg atoms with optical lattices.

The trapping of cold Rydberg atoms advances the spectroscopic study and the manipulation of these exotic atoms, due to the long atom-field interaction times afforded by atom traps. The current types of traps include strong static electric-field 
traps [14], strong static magnetic-field traps [15] (first demonstrated in our group), and optical traps. For the case of static field traps, the trapping is based on lowfield seeking Rydberg states. The spatial confinement of the Rydberg atom in a static field trap depends on the scale of the field gradient, which is determined by the geometry of the electrode package or the magnetic coils which provide the static fields. For the case of optical traps, the trapping force is provided by the gradient of a typically focused laser beam, which can vary over a much smaller length scale than the DC fields generated by machined electrodes or coils. Therefore optical traps usually allow for larger trapping forces at a given potential depth than static-field traps. Conversely, optical traps allow for the realization of a given trapping force with much weaker trapping potentials than static-field traps. Also, the static field create massive energy level shifts of the atomic levels, while lattice light shift is minimal compared to static field traps.

In our group, we utilize the standing-wave pattern of optical lattices to manipulate Rydberg atoms. A ponderomotive optical lattice ( $(\mathrm{POL})$ is an optical-lattice trap for Rydberg atoms, which utilizes the ponderomotive energy shift [16 18] of the quasifree electron to form modulated trapping potentials for Rydberg atoms. In the case of a one-dimensional optical lattice along the z-axis, the ponderomotive potential is

$$
V_{p}(z)=\frac{e^{2} E_{0}^{2}}{4 m_{e} \omega^{2}}[1+\cos (2 k z)],
$$

where $E_{0}$ is the amplitude, $k$ is the wavenumber, and $\omega$ is the angular frequency of the laser light field. The ponderomotive optical lattice trap for Rydberg atoms was proposed by our group in 2000 [19], and has been experimentally demonstrated after ten years of work [20, 21]. Other studies based on the POL can be found in references [22, 23].

The effort to study ponderomotive lattices in our group expanded into two direc- 
tions after the experimental demonstration: one of the directions is to drive Rydberg transitions by amplitude modulation of the lattice with a modulation frequency given by the frequency of the desired transition. This fundamentally new type of spectroscopy method allow us with spatial control of microwave Rydberg transition. The recent experimental demonstration can be found in reference [24]. The long-term goal is the high-precision measurement of Rydberg constant via this lattice-amplitudemodulation magic transition between circular Rydberg states.

The other effort is the study of the adiabatic trapping potentials of Rydberg atoms in a deep $1064 \mathrm{~nm}$ ponderomotive optical lattice [25], which have not yet been probed. The trapping potential seen by the Rydberg atom is an average of the standing wave pattern of the free-electron ponderomotive trapping potential, using the Rydberg electron wavefuntion as a weighting factor. We predict rich trapping structures due to large light shifts, then the lattice-mixed hydrogenic states should become accessible through two-photon Rydberg excitation. In this deep lattice, near the lattice inflection point the lattice-mixed level structure mimics the level structure caused by the shift of an external electric field, whereas near lattice nodes/antinodes the structure can be described by an effective magnetic field. The effective electric/magnetic field patterns are arranged inside each lattice period, and repeat themselves over the lattice potential wells.

The study requires a high $\mathrm{cw}$ lattice laser intensity of about $20 \mathrm{MW} / \mathrm{cm}^{2}$, which is difficult to obtain in a vacuum cold-atom experiment via direct application of a (narrowband) fiber laser. I took the challenge to build a near-concentric cavity experimental setup for the study of the adiabatic trapping potential. In most of the applications of cavities in laser-cooling experiments, a confocal cavity or nearplanar cavity with extremely high finesse is used. For our experimental requirement, a moderately high finesse and a focal spot at the center of the near-concentric cavity are critical in realizing the high intensity required for the study of a deep ponderomotive 
optical lattice.

The trapping of atom with a very deep optical lattice is somewhat different than the trapping of atoms in a shallow lattice. For the case of a shallow lattice, the loading of ground-state atoms is achieved by overlapping the MOT with the lattice light field. Atoms roll into the bottom of the trap as long as they get MOT laser cooled. The subsequent production of Rydberg atoms is done by a slightly frequency-detuned two-photon excitation, where the laser detuning compensate the lattice-induced light shift. To load atoms into a deep optical lattice, we start the loading of groundstate atoms into a shallow lattice. Then, we perform lattice adiabatic compression to compress the atom cloud to the bottom of a deep optical lattice, where the atoms see large light shift. The following Rydberg excitation relies on a careful adjustment of the excitation lasers to excite the atoms resonantly through the strongly light-shifted intermediate $5 \mathrm{P}_{3 / 2}$ state. The $5 \mathrm{P}_{3 / 2}$ light shifts are on the order of several $\mathrm{GHz}$ in my optical lattice; in addition, the levels are split by the tensor polarizability.

It worth to stress the idea of deep-lattice experiment again: with the nearconcentric cavity, we mean to harness the atoms with massive light shift until atomic energy levels are mixed and the high-angular momentum (hydrogenic) states become accessible to regular two-photon Rydberg excitation. This aspect differs from most other work done with optical lattices. The spatial variation of the cavity-generated light shift is dramatic, all the rich, lattice-mixed, hydrogenic-state structures repeat themselves on the scale of the lattice laser wavelength. The structures resulted from the nonlinear response of Rydberg atoms to the trapping potential would be useful for the future quantum manipulation experiments. In addition to the study of lattice-mixed Rydberg hydrogenic state, the cavity-generated optical traps are suitable for a.c. polarizability measurement of excited atomic states, for the merit of the outstanding light shift.

Another merit out of the cavity is its nearly perfect trapping geometry. The 
cavity is sealed inside vacuum and no other elements in the way of the circulating laser power. As a result, the cavity generated optical trapping potentials only depends on the cavity modes. The cavity functions like a mode filter - it "cleans" the input laser and guarantees perfect cavity modes inside the cavity. In addition, due to the long Rayleigh length of the focus at the cavity center, the trap results in the formation of very dense, long and slim atom samples that can be used for a variety of cold-atom experiments, such as search for new Rydberg molecules and Rydberg electromagnetically induced transparency (EIT).

The objective of my thesis work is to build a new laser-cooling experimental apparatus with the near-concentric cavity as the core of the experiment. I also developed the adiabatic compression method to actually trap cold atom with high laser intensity. In the following chapters, I elaborate on my experimental work and the initial experimental results. The following is an outline of this thesis.

- In Chapter II] I give a summary of the optics work that is related to the designing of the cavity experiment.

- In Chapter III, I provide the technical details of this newly-built experiment.

- In Chapter IV, I review the preliminary results of the cavity experiment.

- In Chapter V, I discuss the critical experimental work that allows us to generate the large light shift with the cavity setup.

- In Chapter V, VI, and VIII, I present the recent result of the cavity-generated deep optical-lattice experiments.

- I summarize the accomplishment of this thesis work and outline the future direction of this project in Chapter IX. 


\section{CHAPTER II}

\section{Near-concentric cavity}

The mirror surfaces of a two-mirror optical cavity define the boundary of the cav-

ity. Laser light traveling inside the cavity with the wavefront matching the mirror surfaces gets retro-reflected onto itself. A general mathematical analysis based on the ray reflection by cavity mirrors leads to a simple geometrical parameter, the "resonator g parameter" [2], that describes the properties of a cavity. In Sec. 2.1, I provide the details of the g-parameter analysis. In Sec. 2.1.2, I summarize the g-parameter analysis for a near-concentric cavity, which is also the core of my experimental apparatus. In Sec. 2.2 and 2.3 , I discuss the experimental realization of a near-concentric cavity.

\subsection{Stability analysis}

Fig. 2.1 shows an optical cavity composed of two mirrors centered on the z-axis, mirror $1\left(\mathrm{M}_{1}\right)$ and mirror $2\left(\mathrm{M}_{2}\right)$. The positions of the mirror surfaces of $\mathrm{M}_{1}$ and $\mathrm{M}_{2}$ on the $\mathrm{z}$-axis are $z_{1}$ and $z_{2}$, respectively. The cavity length $L$ is calculated as $z_{2}-z_{1}$.

A Gaussian beam traveling inside this two-mirror cavity is retro-reflected onto itself by the cavity mirrors when the radii of curvature of its wavefront match the radii of curvature of the cavity mirror surfaces. There exists a specific mirror configuration, i.e. mirror surfaces at $z_{1}$ and $z_{2}$, such that the Gaussian beam gets retro-reflected 


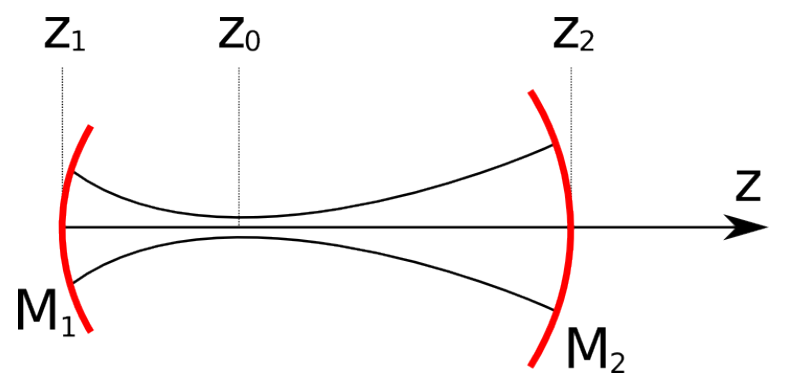

Figure 2.1: Gaussian beam inside a two-mirror optical cavity. The propagating direction is along z-axis. Red curves: mirror surfaces of mirror $1\left(\mathrm{M}_{1}\right)$ and mirror $2\left(\mathrm{M}_{2}\right)$.

by the mirror surfaces multiple times instead of leaving the cavity. The circulating laser light inside the cavity forms a stable waist $w_{0}$ at $z_{0}=0$. The configuration of the cavity mirrors hence defines the boundary condition for the laser light to oscillate inside the cavity. The above description is mathematically expressed by,

$$
\begin{aligned}
& R\left(z_{1}\right)=z_{1}+\frac{z_{R}^{2}}{z_{1}}=-r_{1}, \\
& R\left(z_{2}\right)=z_{2}+\frac{z_{R}^{2}}{z_{2}}=r_{2}
\end{aligned}
$$

where $r_{1}$ and $r_{2}$ are the radii of curvature of $\mathrm{M}_{1}$ and $\mathrm{M}_{2}$, respectively, and $z_{R}$ is the Rayleigh length of the Gaussian beam, $z_{R}=\frac{n \pi w_{0}^{2}}{\lambda}$.

Given a pair of cavity mirrors with known radii of curvature, we would like to know which cavity configuration leads to a stable waist inside the cavity, and the size of the waist. That is, we would like to find the expressions of $z_{1}, z_{2}$ and $w_{0}$ in terms of known values $r_{1}, r_{2}$, and the cavity length $L$. To begin with, Eq. 2.1 and the cavity length satisfy,

$$
\begin{aligned}
\frac{z_{R}^{2}}{z_{1}} & =-r_{1}-z_{1} \\
\frac{z_{R}^{2}}{z_{2}} & =r_{1}-z_{2} \\
z_{2} & =z_{1}+L .
\end{aligned}
$$


$z_{1}, z_{2}$ and $z_{R}$ follow,

$$
\begin{aligned}
z_{1} & =\frac{-L\left(r_{2}-L\right)}{r_{1}+r_{2}-2 L} \\
z_{2} & =\frac{L\left(r_{1}-L\right)}{r_{1}+r_{2}-2 L} \\
z_{R}^{2} & =\frac{L\left(r_{1}-L\right)\left(r_{2}-L\right)\left(r_{1}+r_{2}-L\right)}{\left(r_{2}+r_{2}-2 L\right)^{2}}
\end{aligned}
$$

The waist $w_{0}$ is calculated from the Rayleigh length $z_{R}$,

$$
\begin{aligned}
w_{0} & =\sqrt{\frac{\lambda z_{R}}{n \pi}} \\
& =\sqrt{\frac{\lambda}{n \pi}}\left[\frac{L\left(r_{1}-L\right)\left(r_{2}-L\right)\left(r_{1}+r_{2}-L\right)}{\left(r_{2}+r_{2}-2 L\right)^{2}}\right]^{\frac{1}{4}}
\end{aligned}
$$

where $n$ is the index of refraction inside the cavity, and $\lambda$ is the wavelength of the Gaussian beam.

The use of the "g parameter" simplifies further analysis. A g parameter is defined for each cavity mirror. The value of a g parameter solely depends on the geometrical properties of a cavity.

$$
\begin{aligned}
& g_{1}=1-\frac{L}{r_{1}} \\
& g_{2}=1-\frac{L}{r_{2}}
\end{aligned}
$$

Using the g parameters, $z_{1}, z_{2}$ and $w_{0}$ are written as,

$$
\begin{aligned}
z_{1} & =\frac{-L g_{2}\left(1-g_{1}\right)}{g_{1}+g_{2}-2 g_{1} g_{2}} \\
z_{2} & =\frac{L g_{1}\left(1-g_{2}\right)}{g_{1}+g_{2}-2 g_{1} g_{2}} \\
w_{0} & =\sqrt{\frac{L \lambda}{n \pi}}\left[\frac{g_{1} g_{2}\left(1-g_{1} g_{2}\right)}{\left(g_{1}+g_{2}-2 g_{1} g_{2}\right)^{2}}\right]^{\frac{1}{4}}
\end{aligned}
$$


From the propagation of the Gaussian beam, the waist $w_{1}$ and $w_{2}$ at the mirror surfaces of $\mathrm{M}_{1}$ and $\mathrm{M}_{2}$ are,

$$
\begin{aligned}
w_{1} & =w_{0} \sqrt{1+\frac{z_{1}^{2}}{z_{R}^{2}}} \\
& =\sqrt{\frac{L \lambda}{n \pi}}\left[\frac{g_{2}}{g_{1}\left(1-g_{1} g_{2}\right)}\right]^{\frac{1}{4}} \\
w_{2} & =\sqrt{\frac{L \lambda}{n \pi}}\left[\frac{g_{1}}{g_{2}\left(1-g_{1} g_{2}\right)}\right]^{\frac{1}{4}}
\end{aligned}
$$

Based on the expressions of $w_{1}$ and $w_{2}$, a two-mirror cavity has physical values of $w_{1}$ and $w_{2}$ when $0<g_{1} g_{2}<1$. A cavity that satisfies this condition is a stable cavity, which keeps laser light retracing itself without walking off from the cavity axis. If $g_{1} g_{2}<0$ or $g_{1} g_{2}>1$, the cavity is unstable. If $g_{1} g_{2}=0$ or $g_{1} g_{2}=1$, the cavity is called "marginally stable".

Fig. 2.2 shows a plot of $g_{1} g_{2}$ and a few examples of two-mirror cavities. Cavities inside the blue-shaded region are stable. Cavities outside the blue-shaded region are unstable. Cavities on the boundary $g_{1} g_{2}=0$ or $g_{1} g_{2}=1$ are marginally stable. The red dashed line on the plot marks cavities with $g_{1}=g_{2}$, i.e. symmetric two-mirror cavities. There are three examples of cavities on this red dashed line: plane-parallel (planar) cavity, confocal cavity, and concentric cavity. Confocal cavities are pretty much the most common among these three cavities. Occasionally, people are confused by the naming, i.e. confocal cavity vs. concentric cavity. The difference between confocal cavities and concentric cavities is the cavity length. The cavity length of symmetric confocal cavities is $r$, because the center of curvature of each cavity mirror is on the center of the surface of the other cavity mirror. With the same pair of cavity mirrors, the cavity length of a symmetric concentric cavity is $2 r$. The centers of curvature of both mirrors overlap at the cavity center for a concentric cavity.

Practically speaking, the difference between a concentric cavity and a confocal 
cavity is that a concentric cavity is frustrating from a graduate student's point of view. This can be explained by the stability diagram. Starting with the confocal configuration $(L=r)$, following the red dashed line and moving toward the concentric configuration $(L=2 r)$, the beam waist $w_{0}$ at the cavity center is narrowing down as the cavity mirrors are brought further away from each other. As the cavity length increases and the waist decreases, the cavity becomes more sensitive to angular misalignment. At the concentric configuration, both cavity mirrors focus exactly at the same spot. Any angular misalignment can fail this "point contact", break the round trip of the circulating light and throw the cavity into the unstable regime on the stability diagram.

Despite the experimental challenges that concentric cavities possess, particularly if used in ultra-high vacuum conditions as it is the case in our experiment, they present

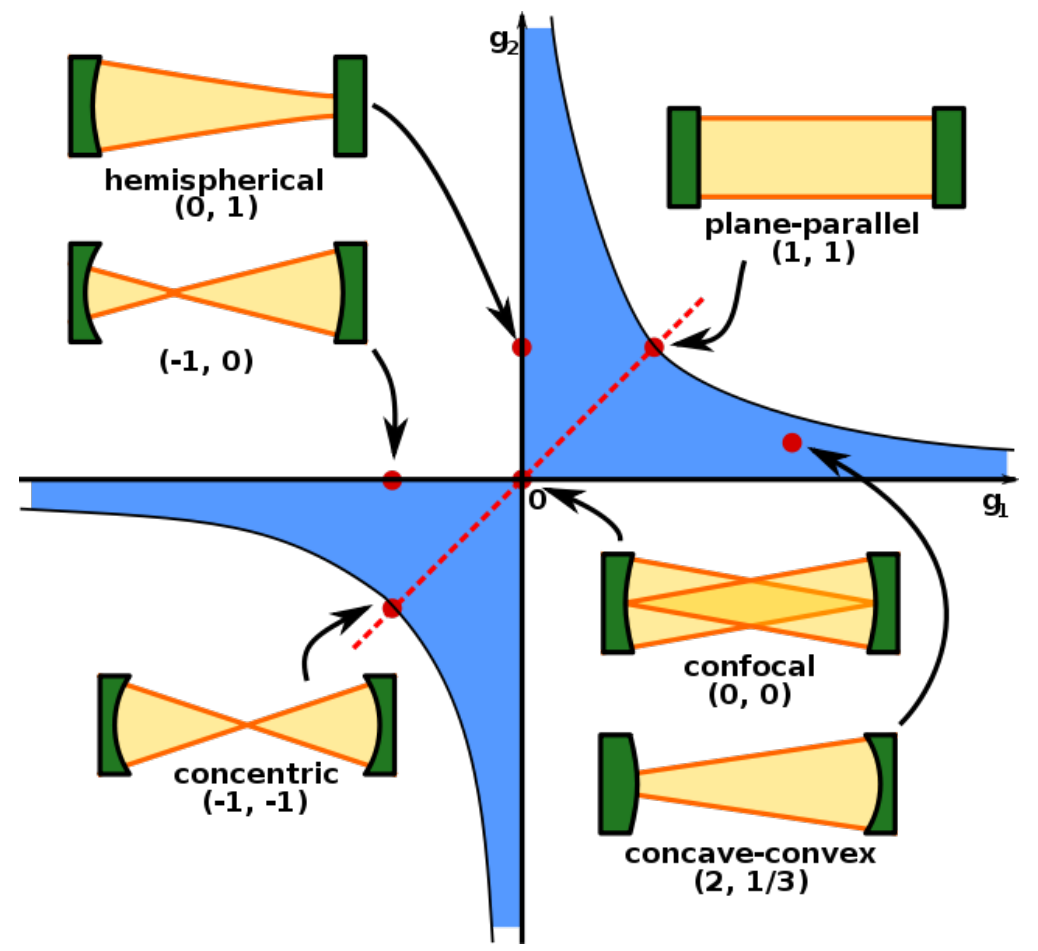

Figure 2.2:

Stability diagram of two-mirror cavities. Origin: wikipedia
(http://commons.wikimedia.org/wiki/File:Laser_resonator_
stability.svg)


many worthwhile advantages. In the beginning, the critical reason that pushed us to design such a in-vacuum concentric cavity experiment is that we need high laser intensity at the center where the cold atom cloud is located, and low intensity at the mirror surfaces. Among all of the symmetric, stable or marginally stable two-mirror cavities, the concentric cavity is the only configuration that has a focus at the cavity center, which ensures high laser intensity. In this type of cavity, there is also much less chance of damaging the cavity mirror coatings in a vacuum environment. We have realized that there are even more advantages of using a concentric configuration, which will be discussed in our findings in later chapters.

\subsubsection{Cavity modes}

The electric field of a Gaussian beam propagating in z-axis in free-space can be described by Hermite polynomials, $H_{\mathrm{n}}$, as follows:

$$
\begin{aligned}
& E_{\mathrm{mn}}(x, y, z)= \\
& E_{0} \frac{w_{0}}{w} H_{\mathrm{m}}\left(\frac{\sqrt{2} x}{w}\right) H_{\mathrm{n}}\left(\frac{\sqrt{2} y}{w}\right) \exp \left[-\left(x^{2}+y^{2}\right)\left(\frac{1}{w^{2}}+\frac{i k}{2 R(z)}\right)-i k z+i(\mathrm{~m}+\mathrm{n}+1) \zeta(z)\right]
\end{aligned}
$$

where $R(z)$ is the radius of curvature of the wavefront, $w=w(z)$ is the radius of the beam, measured from the z-axis to the position where the field amplitude drops to $\frac{1}{e}$ of the axial value, and $\zeta(z)=\tan ^{-1} \frac{z}{z_{R}}$ is the Guoy phase shift. $\mathrm{m}$ and $\mathrm{n}$ are the orders of Hermite polynomials which describe the electric field in x-axis and y-axis, respectively. At the lowest order, $\mathrm{m}=0$ and $\mathrm{n}=0$, the transverse profile is the familiar Gaussian beam profile. At higher orders, the profile is composed of lobes of rectangular symmetry. mth order refers to $\mathrm{m}$ nulls and $\mathrm{m}+1$ peaks in the $\mathrm{x}$-axis. For example, the profile of a mode with $\mathrm{m}=1, \mathrm{n}=1$ has 2 by 2 , four bright lobes.

For a Gaussian beam to oscillate within the cavity while retaining its profile, the 
single-pass phase shift experienced by the beam has to be a multiple of $\pi$,

$$
\begin{aligned}
\phi\left(z_{2}-z_{1}\right) & =\mathrm{q} \pi \\
& =k L-(\mathrm{m}+\mathrm{n}+1)\left(\tan ^{-1} \frac{z_{2}}{z_{R}}-\tan ^{-1} \frac{z_{1}}{z_{R}}\right) \\
& =k L-(\mathrm{m}+\mathrm{n}-1) \cos ^{-1} \sqrt{g_{1} g_{2}}
\end{aligned}
$$

So the frequencies of self-reproducing, Hermite-gaussian eigenmodes within the cavity are,

$$
\begin{aligned}
& k_{\mathrm{qmn}}=\frac{\pi}{L}\left[\mathrm{q}+(\mathrm{m}+\mathrm{n}+1) \frac{\cos ^{-1} \sqrt{g_{1} g_{2}}}{\pi}\right] \\
& \nu_{\mathrm{qmn}}=\frac{c}{2 n L}\left[\mathrm{q}+(\mathrm{m}+\mathrm{n}+1) \frac{\cos ^{-1} \sqrt{g_{1} g_{2}}}{\pi}\right]
\end{aligned}
$$

The above analysis gives the frequencies of the Hermite-Gaussian (HG) cavity eigenmodes. Similar analysis can also be done with Laguerre-Gaussian beams of cylindrical symmetry, or Ince-Gaussian beams of elliptical symmetry [26, 27]. The figure on the title page is a sample of the cavity modes captured by an infrared camera. I took these picture by "mis-aligning" the cavity mirrors. It seems fairly easy to obtain cavity modes of all the three types of symmetries with a concentric cavity. Although the Hermite-gaussian cavity modes are of the most interest for the atom-trapping experiments in this thesis work, one should keep in mind that the work can be extended by using cavity modes of cylindrical or elliptical symmetry.

The analysis here has essentially no difference compared to a quantum mechanics problem. The cavity mirrors define the boundary condition, and we try to find the eigenbasis of cavity modes. The cavity exhibits discrete cavity modes. The laser intensity profile of a cavity modes is "quantized" in both transverse and axial directions. In the axial direction, the modes generate periodic lightshift for cold-atoms, mimicking the periodic lightshift of standing waves formed by counter-propogating 
laser beams. Together with the transverse cavity modes, the cavity is able to generate three-dimensional optical trapping potentials.

Table 2.1:

summary of two-mirror cavity parameters. Further details can be found in Ref.[2].

\begin{tabular}{l|l}
\hline g parameters & $g_{1}=1-\frac{L}{r_{1}}, g_{2}=1-\frac{L}{r_{2}}$ \\
beam waist at cavity center & $w_{0}=\sqrt{\frac{L \lambda}{n \pi}}\left[\frac{g_{1} g_{2}\left(1-g_{1} g_{2}\right)}{\left(g_{1}+g_{2}-2 g_{1} g_{2}\right)^{2}}\right]^{\frac{1}{4}}$ \\
beam waist at $\mathrm{M}_{1}$ & $w_{1}=\sqrt{\frac{L \lambda}{n \pi}}\left[\frac{g_{2}}{g_{1}\left(1-g_{1} g_{2}\right)}\right]^{\frac{1}{4}}$ \\
beam waist at $\mathrm{M}_{2}$ & $w_{2}=\sqrt{\frac{L \lambda}{n \pi}}\left[\frac{g_{1}}{g_{2}\left(1-g_{1} g_{2}\right)}\right]^{\frac{1}{4}}$ \\
frequencies of cavity modes & $\nu_{\mathrm{qmn}}=\frac{c}{2 n L}\left[\mathrm{q}+(\mathrm{n}+\mathrm{m}+1) \frac{\cos ^{-1} \sqrt{g_{1} g_{2}}}{\pi}\right]$ \\
axial mode splitting & $\Delta \nu_{\mathrm{ax}}=\frac{c}{2 n L}$ \\
transverse mode splitting & $\Delta \nu_{\text {trans }}=\frac{c}{2 n L}\left[\frac{\cos ^{-1} \sqrt{g_{1} g_{2}}}{\pi}\right]$ \\
\hline
\end{tabular}

\subsubsection{Near-concentric cavity}

The perfect concentric configuration, $L=2 r$, is not experimentally feasible, due to its exaggerated sensitivity to angular misalignment. To integrate a concentric configuration into a real experiment, we reduce the cavity length by a tiny amount

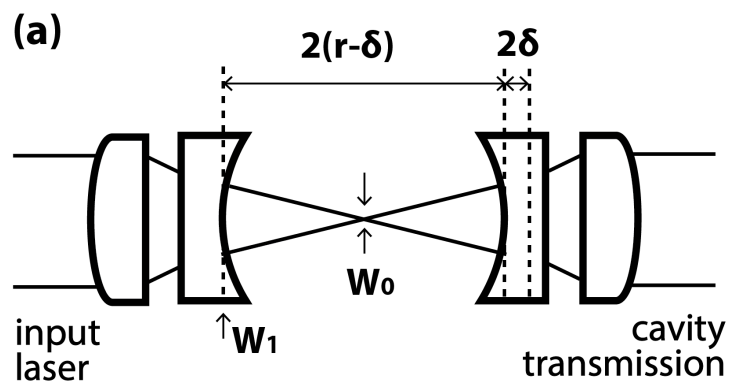

(b)

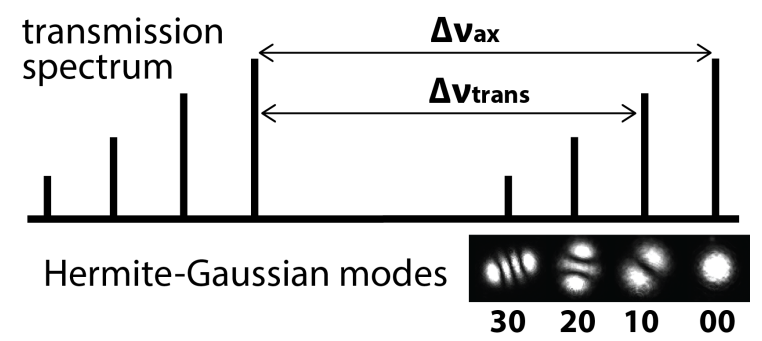

Figure 2.3:

(a) Near-concentric cavity. A pair of coupling lenses is required to focus a collimated input beam at the cavity center. See Sec. 2.2 for more information. (b) Cavity transmission spectra illustration and the mostly seen four Hermite-Gaussian ( $\mathrm{HG}$ ) cavity modes. 
$\delta$. The reduced cavity ensures that the cavity stays inside the stable regime. For a symmetric near-concentric cavity, $r_{1}=r_{2}=r, L=2(r-\delta), g_{1}=g_{2}=g$. The cavity parameters in Table 2.1 can be approximated by,

$$
\begin{aligned}
w_{0} & \approx \sqrt{\frac{r \lambda}{\pi}}\left[\frac{\delta}{r}\right]^{1 / 4}, \\
w_{1} & \approx \sqrt{\frac{r \lambda}{\pi}}\left[\frac{r}{\delta}\right]^{1 / 4}, \\
\nu_{\mathrm{qmn}} & \approx \frac{c}{4 r}\left[\mathrm{q}+(\mathrm{n}+\mathrm{m}+1)\left(1-\frac{2}{\pi} \sqrt{\frac{\delta}{r}}\right)\right], \\
\Delta \nu_{\mathrm{ax}} & =\frac{c}{4 r} \\
\Delta \nu_{\text {trans }} & =\frac{c}{4 r}\left(1-\frac{2}{\pi} \sqrt{\frac{\delta}{r}}\right) .
\end{aligned}
$$

where the index of refraction is 1 in vacuum. Fig. 2.3 illustrates the geometry and cavity transmission spectrum of a near-concentric cavity. In Fig. 2.3(b), the cavity is aligned in a manner such that it slightly deviates from cylindrical symmetry. This alignment lifts the degeneracy of the Hermite-Gaussian cavity modes in the $\mathrm{x}$ - and $\mathrm{y}$ axes, allowing easy access to individual HG cavity modes.

Empirically, the more the cavity length is reduced, the easier it is to align the cavity. This can be pictured by the overlapping of the beam focus inside the cavity. For a perfect concentric configuration, the tips of the two retro-reflected light cones from the two cavity mirrors have to overlap at exactly the same point. The round-trip path of the circulating light will be broken by minute misalignment. However, if the cavity length is reduced, there will still be a good chance that the two light cones overlap in case of small angular misalignment due to a larger beam waist at that point. In the language of $g_{1} g_{2}$, as the cavity length is reduced, the concentric cavity moves toward the confocal cavity limit on the stability diagram. The cavity starts acquiring the properties of a confocal cavity - fairly insensitive to angular misalignment and very user-friendly. 
The in-vacuum near-concentric cavity is described in in detail Sec. 3.1 . The $\frac{\delta}{r}$ is about 0.004 in the current setup. This is measured by scanning the cavity length and calculating the percentage splitting of $(\mathrm{q}-1,1,0)$ to $(\mathrm{q}, 0,0)$ cavity mode relative to $\Delta \nu_{\mathrm{ax}}$, which is about $4 \%$ (see Fig. 2.3). The beam waist at the cavity center is calculated to be $23 \mu \mathrm{m}$. Since $\frac{w_{1}}{w_{0}}=\sqrt{\frac{r}{\delta}}$, the intensity ratio scales to $\frac{r}{\delta}$. The intensity is increased by 250 times at the cavity center. The large waist ratio not only allows us a high laser intensity at the cavity center, but also reduces the chance of damaging the mirror surface. In comparison, a confocal cavity would have a waist ratio of only $\sqrt{2}$.

Fig. 2.4 is an illustration of the transmission spectra of the three symmetric-two mirror cavity: near-planar(plane-parallel), confocal, and near-concentric. The cavities are assumed to have the same cavity length in the drawing. Both the near-planar and

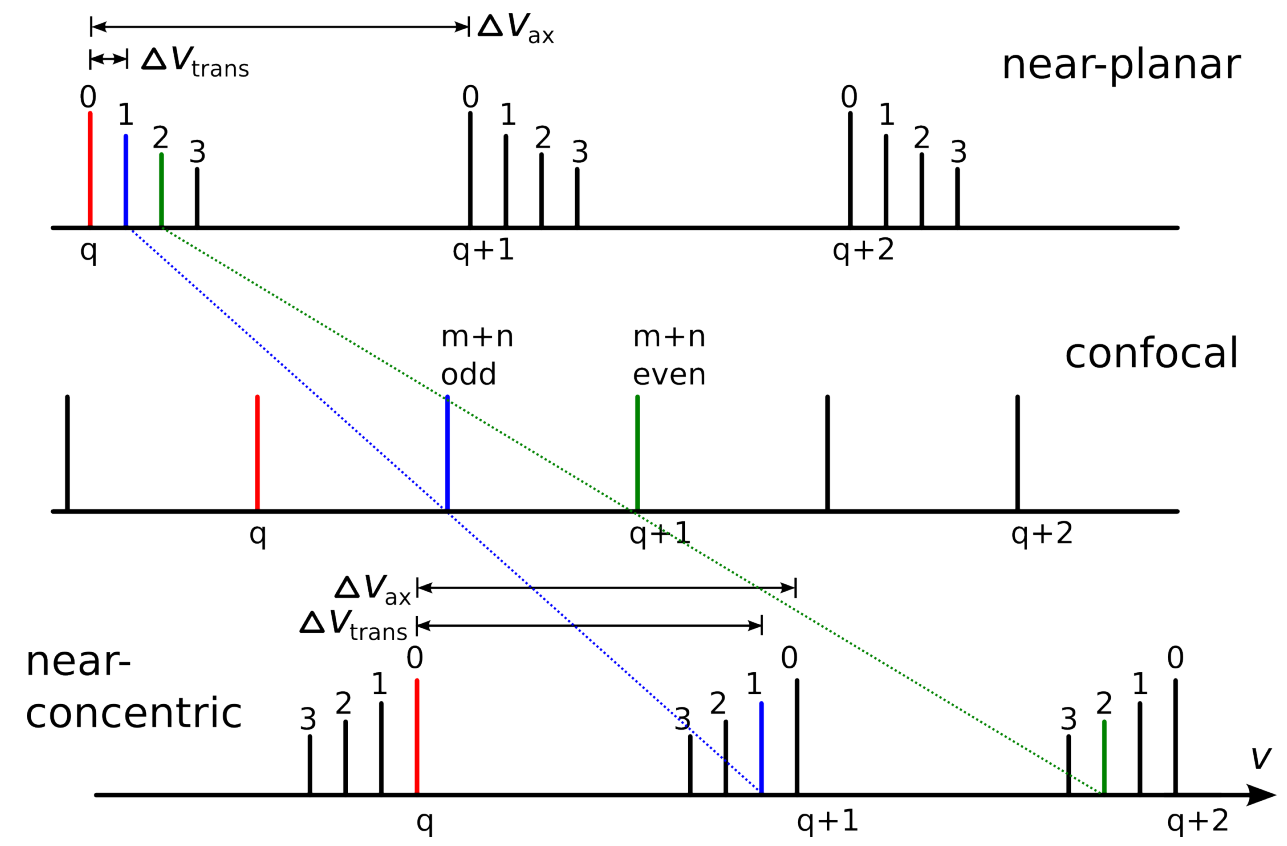

Figure 2.4:

Illustration of transmission spectra for near-planar, confocal, and nearconcentric cavities with the same cavity length. near-planar: $g_{1}, g_{2} \rightarrow 1^{-}$, $\frac{\cos ^{-1} \sqrt{g_{1} g_{2}}}{\pi} \rightarrow 0^{+}$. confocal: $g_{1}, g_{2}=0, \frac{\cos ^{-1} \sqrt{g_{1} g_{2}}}{\pi}=\frac{1}{2}$. near-concentric: $g_{1}, g_{2} \rightarrow-1^{+}, \frac{\cos ^{-1} \sqrt{g_{1} g_{2}}}{\pi} \rightarrow 1^{-}$. The cavity modes for both near-planar and near-concentric cavities are non-degenerate, while the cavity modes of a confocal cavity are degenerate. 
near-concentric cavities have non-degenerate cavity modes, while the cavity modes of confocal cavity is degenerate. Because of the degeneracy, the Free spectral range $(\underline{\mathrm{FSR}})$ of a confocal cavity is calculated as $\frac{1}{2} \Delta \nu_{\mathrm{ax}}=\frac{c}{4 n L}$. For the case of nearconcentric cavities, we use $\mathrm{FSR}=\Delta \nu_{\mathrm{ax}}=\frac{c}{2 n L}$.

\subsection{Optical near-concentric cavity design}

\subsubsection{Coupling lens}

In reality, a pair of coupling lenses is required for the near-concentric cavity. The lens at the entrance focuses the input laser at the cavity center. The lens at the exit collimates the transmitted light for observation. The placement of the coupling lens is critical to couple a large portion of the incident laser power into selected cavity modes.

In the experimental setup, the cavity is composed of two cavity mirrors with $r=25 \mathrm{~mm}$. The focal length of the pair of focusing lens is $40 \mathrm{~mm}$. The focusing lenses are relatively thin GRADIUM@ lenses, while the thickness of the substrate of the cavity mirrors is $0.375 "$. The position of the lenses is calculated by assuming a collimated input beam that focuses at the cavity center. The position of optimal

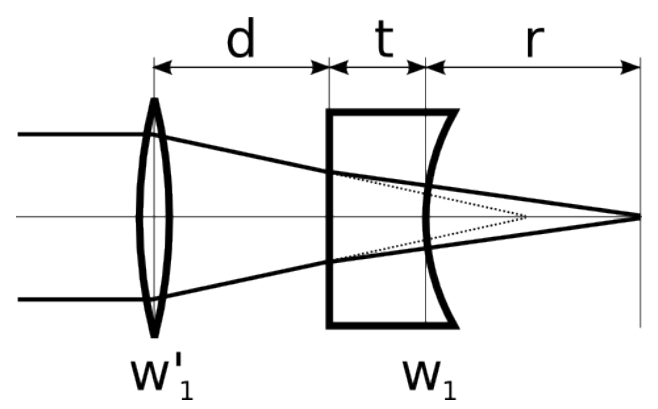

Figure 2.5: Focusing collimated input beam into a thick cavity mirror with a thin coupling lens. $w_{1}$ is the waist of the Gaussian cavity mode $\left(\mathrm{HG}_{00}\right)$ on the cavity mirror surface. $w_{1}^{\prime}$ is the waist on the thin focusing lens. 
coupling is approximated by,

$$
d \simeq f-\frac{t+r}{n}
$$

where $n$ is the refractive index of the mirror substrate, and the other variables are shown in Fig. 2.5. The optimal position $d$ is $17.63 \mathrm{~mm}$ for the current cavity setup.

\subsubsection{Cavity mode-matching}

The cavity functions like a mode filter as follows: the cavity extracts the portion of input light matching the cavity mode at the cavity mirror surfaces and rejects everything else (see Fig. 2.6). Inside the cavity, the modes of the light field are nearly perfect, which can be verified by monitoring the transmitted light. As a result, the cavity guarantees nearly perfect optical trapping potential for cold atoms.

In order to maximize the usage of input laser power by the cavity, the beam waist of the input laser is adjusted to enhance the mode-matching to the Gaussian cavity mode. The optimal input beam waist is the waist that matches the waist on the focusing lens. The waist at the focusing lens is then focused down to match the waist of the cavity mode at the cavity mirror surface. The relation between the input beam

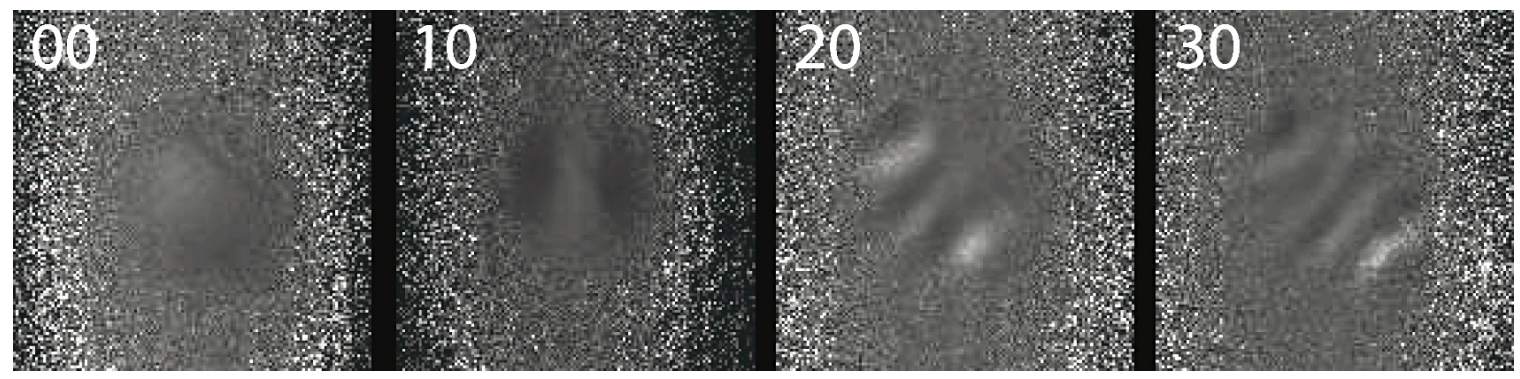

Figure 2.6:

Profiles of the laser beam rejected by the cavity when the cavity is locked to Hermite-Gaussian ( $\mathbf{H G}$ ) 00, 10, 20, and 30 modes. The input beam is Gaussian. The cavity extracts the portion of input which matches the cavity modes and then rejects the rest, leaving dark strips on the profile of the return beam. 
waist and the cavity mode waist at the mirror surfaces is,

$$
w_{1}^{\prime} \simeq w_{1}\left(\frac{t+r+n d}{r}\right) \simeq 2.411 w_{1}
$$

The waist $w_{1}$ is calculated using Eq. 2.18 with the ratio $\frac{\delta}{r}$ obtained from the cavity transmission spectra. Beam-profiling the transmitted laser at the focusing lens also gives the $w_{1}^{\prime}$. For the current setup, the input laser beam is resized to a waist about $900 \mu \mathrm{m}$; this waist is the closest to the optimal Gaussian mode-matching waist we can obtain, due to the limited options of telescope lenses.

\subsubsection{Theoretical coupling efficiency}

The finesse $\mathcal{F}$ of a cavity is defined as the FSR divided by the full-width-halfmaximum ( $(\underline{\mathrm{FWHM}})$. The transmitted power, which can be easily measured, times $\frac{\mathcal{F}}{\pi}$ gives an estimate of the circulating laser power inside the cavity. The theoretical

maximal value of $\mathcal{F}$ equals $\frac{\pi \sqrt{R}}{1-R}$, where $R$ is the reflectivity of the cavity mirror. For the cavity mirrors in my setup, the reflectivity is guaranteed to be more than $99.5 \%$ by the manufacturer, so the theoretical limit of $\mathcal{F}$ is 626 . The $\mathcal{F}$ measured from the transmitted spectra is around 600, so the power inside the cavity is about 200 times of the transmitted power.

The finesse $\mathcal{F}$ of the cavity is moderately high compared to the extremely high $\mathcal{F}$ in a cavity QED experiment, which is usually at least several thousand. In principle, the mirrors for a very high $\mathcal{F}$ cavity are commercially available by customizing the thin-films coatings of dielectric materials on the glass mirror substrate. Nevertheless, the moderately high $\mathcal{F}$ has advantages for the purpose of getting better coupling efficiency and higher circulating laser power. The moderately high $\mathcal{F}$ also makes the cavity easier to be aligned, and stabilized by electronic feedback circuits.

The coating on the mirror surfaces reflects most of the injected laser light, with a 
tiny amount of light getting transmitted through. In reality, the coatings also cause a finite amount of absorption or scattering loss. The scattering loss is the familiar phenomenon that the coating of a mirror always seems lit-up when a strong beam hits the mirror surface.

To obtain the theoretical coupling efficiency, the cavity mirror is again treated as a boundary for the electric field of a Gaussian cavity mode. The reflected and transmitted electric field is calculated. The power ratios of laser light transmitted, reflected and absorbed by a cavity are,

$$
\begin{aligned}
P_{\text {transmitted }} & =\frac{T^{2}}{(1-R)^{2}} P_{\text {input }} \\
P_{\text {reflected }} & =\frac{R A^{2}}{(1-R)^{2}} P_{\text {input }} \\
P_{\text {loss }} & =\frac{A(1-R)^{2}+A T+A R T}{(1-R)^{2}} P_{\text {input }}
\end{aligned}
$$

where $R$ is the reflectivity, $T$ is the transmission, and $A$ is the loss of the mirror coating. $A+T+R=1$. In order to get high intensity inside the cavity, the reflectance of a mirror is not the only factor that needs to be considered. The tiny amount of transmission and absorption loss dramatically affects the coupling efficiency.

The values of $A$ and $T$ for the cavity mirrors are not provided by the manufacturer, but I measured them to be $0.28 \%$ and $0.14 \%$, respectively. This puts the theoretical maximal coupling efficiency of the cavity to $11 \%$ for the Gaussian cavity mode. The real coupling efficiency is usually a bit lower, due to the input laser mode is not perfectly matching the cavity mode.

\subsection{Cavity stabilization with Pound-Drever-Hall scheme}

A tiny portion of the circulating laser light inside the cavity gets transmitted through the cavity mirror at the exit side, providing us an non-invasive way to ob- 
serve the field inside the cavity. We would like to stabilize the cavity such that the transmitted power is at its peak value, which guarantees that the intracavity power is also stabilized to the maximal value.

If the input laser frequency is modulated near a resonance, not only the cavity transmission but also the cavity reflection, will contain the information of whether the input laser frequency is above or below the resonance. However, a transmission or reflection spectrum alone is not enough for an electronic feedback circuit (usually a negative feedback circuit, e.g. a proportional-integral-derivative control) to stabilize the laser frequency at the resonance. The Pound-Drever-Hall (PDH) scheme is used to convert the cavity reflection from an absorption peak to a zero-crossing slope that a regular electronic feedback circuit recognizes as an error signal. The negative feedback circuit will try to bring the laser frequency back to the zero-crossing position, which has the same position as the position of peak absorption on the spectrum. In this manner, the circuit can stabilize the laser frequency at the resonance.

The PDH scheme works as follows: the input laser is frequency modulated to create frequency up-shifted and down-shifted sidebands, where the frequency shift is the same as the modulation frequency. The cavity reflection and the modulation frequency are both sent to a mixer, whose output is a multiplication of its inputs. The output of the mixer is filtered by a low pass filter, and the remaining signal is the PDH error signal. Fig. 2.7 is a screenshot showing both the cavity reflection and the PDH error signal.

To compute the PDH error signal we start with the incident electric field $E_{i}=$ $E_{0} \mathrm{e}^{i \omega t}$, which after frequency modulation is,

$$
E_{i}=E_{0} e^{i \omega t} e^{i \beta \sin \Omega t}
$$

where $\beta$ is called modulation depth, and $\Omega$ is modulation frequency. Using Jacobi- 


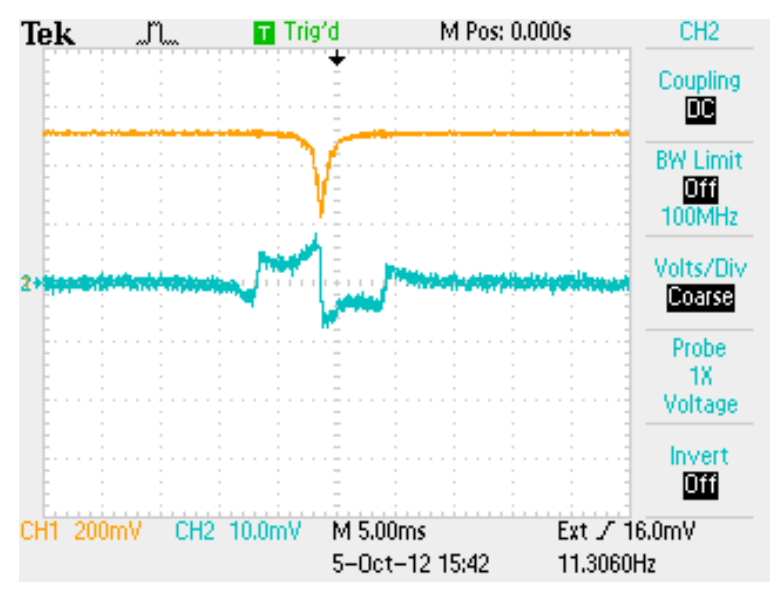

Figure 2.7: A oscilloscope screenshot of the cavity reflection (yellow) and PDH error signal (blue).

Anger expansion,

$$
\begin{aligned}
E_{i} & =E e^{i \omega t}\left(\sum_{\mathrm{n}=-\infty}^{\infty} J_{\mathrm{n}}(\beta) e^{i \mathrm{n} \Omega t}\right) \\
& \approx \underbrace{E_{0} J_{0}(\beta) e^{i \omega t}}_{\text {carrier }}+\underbrace{E_{0} J_{1}(\beta) e^{i(\omega+\Omega) t}-E_{0} J_{1}(\beta) e^{i(\omega-\Omega) t}}_{\text {first-order side bands }}
\end{aligned}
$$

The electric field of the cavity reflection is,

$$
E_{r}=F(\omega) E_{0} J_{0}(\beta) e^{i \omega t}+F(\omega+\Omega) E_{0} J_{1}(\beta) e^{i(\omega+\Omega) t}-F(\omega-\Omega) E_{0} J_{1}(\beta) e^{i(\omega-\Omega) t}
$$

where $F(w)$ is a frequency-dependent complex reflective coefficient of the cavity. On resonances, $\omega$ is an integer multiple of a cavity mode frequency, and $F(\omega)$ has a minimum. The power of cavity reflection is,

$$
\begin{aligned}
P_{r}= & P_{c}|F(\omega)|^{2}+P_{s}|F(w+\Omega)|^{2}+P_{s}|F(w-\Omega)|^{2} \\
& +2 \sqrt{P_{c} P_{s}}\left(\operatorname{Re}\left[F(\omega) F^{*}(\omega+\Omega)\right]-\operatorname{Re}\left[F(\omega) F^{*}(\omega-\Omega)\right]\right) \cos \Omega t \\
& +2 \sqrt{P_{c} P_{s}}\left(\operatorname{Im}\left[F(\omega) F^{*}(\omega+\Omega)\right]+\operatorname{Im}\left[F(\omega) F^{*}(\omega-\Omega)\right]\right) \sin \Omega t \\
& -\sqrt{P_{c} P_{s}}\left(F(\omega+\Omega) F^{*}(\omega-\Omega) e^{2 i \Omega t}+F^{*}(\omega+\Omega) F(\omega-\Omega) e^{-2 i \Omega t}\right)
\end{aligned}
$$


where $P_{c}$ is the carrier power, $P_{s}$ is the sideband power and their sum equals the input laser power $P_{0}$. The power of the first-order side bands is usually on the order of $1 \%$ of $P_{c}$. All the other higher orders, which have much less strength compared to the first order, are neglected. The output signal, together with the modulation frequency is sent to the mixer, and then through the low-pass filter. When the cavity reflection is mixed with a signal $\sin \Omega^{\prime} t$, with oscillation frequency $\Omega^{\prime}=\Omega$, then the term oscillating with $\sin \Omega t$ in the cavity reflection will be filtered out as a DC signal. When the modulation frequency $\Omega$ is higher than several linewidth of the axial mode peaks on the cavity transmission spectra, the cavity reflection is approximated by,

$$
P_{r} \approx 2 P_{s}-4 \sqrt{P_{c} P_{s}} \operatorname{Im}[F(\omega)] \sin \Omega t+f(2 \Omega)
$$

The PDH error signal after the mixer and low pass filter is,

$$
\begin{aligned}
\epsilon & =-4 \sqrt{P_{c} P_{s}} \operatorname{Im}[F(\omega)] \\
& \approx \frac{-4}{\pi} \sqrt{P_{c} P_{s}} \frac{\delta \omega}{\delta \nu},
\end{aligned}
$$

where $\delta \nu$ is the cavity transmission linewidth, and $\delta \omega$ is the difference in frequency from resonance. Reference [28] provides the detailed derivation of the PDH error signal generation.

The PDH error signal has three zero crossing slopes in Fig. 2.7. The zero-crossing position of the large negative slope marks the position of minimal reflection, which is also the position of peak cavity transmission. The other two small slopes with opposite polarity mark the location of first-order side bands. The powers of side bands are so low that they are invisible in the yellow reflection curve.

In the experimental setup, the phase of the input laser is modulated by an electrooptic modulator (EOM) instead of frequency-modulation. The radio frequency ( $(\mathrm{RF})$ modulation amplitude and frequency of the EOM is controlled by a PDH detector 


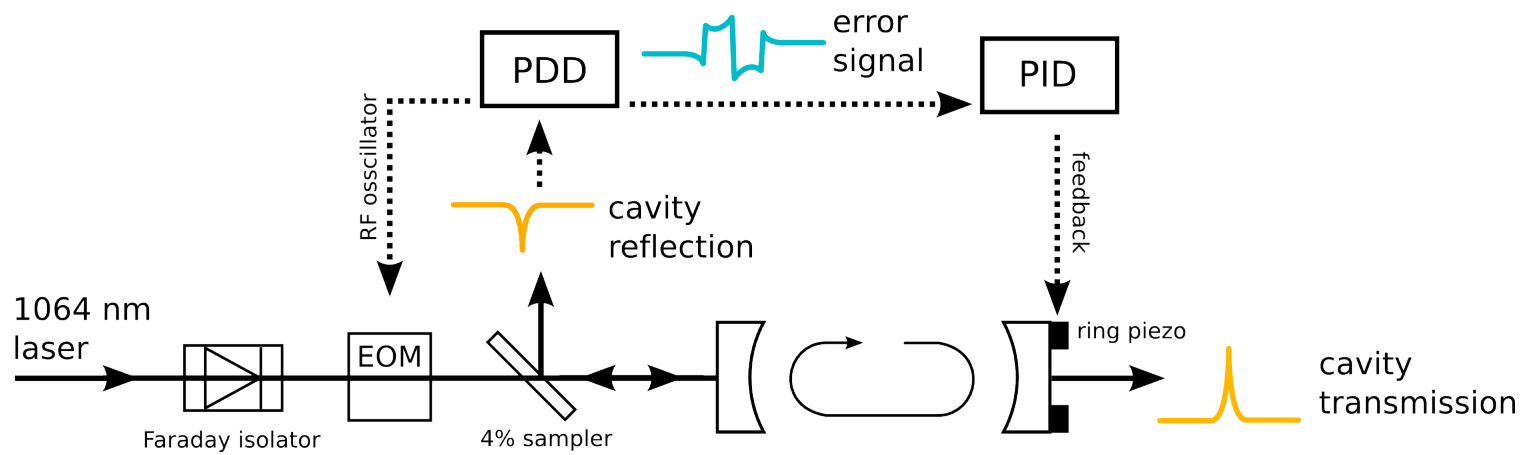

Figure 2.8:

Layout of the Pound-Drever-Hall cavity stabilization with commercial control modules

module (PDD 110, TOPTICA Photonics). The PDD module also takes the cavity reflection and mixes the cavity reflection with its internal oscillator to generate the PDH signal. The PDH error signal is then sent to a PID feedback control module (PID 110, TOPTICA Photonics). The positive high-voltage output of the PID control module is applied to a ring piezo which pushes the cavity mirror to the peak transmission position. We assume that the output of the laser has neither frequency drift nor intensity noise. It is the cavity length which is stabilized relative to the frequency of input laser. See Fig. 2.8 for the layout of the control.

The PDH scheme is fundamental to the cavity stabilization in my experiment. The scheme enables the cavity to peak-lock to a constant laser source. Later on, as the experiment develops, we need the cavity to peak lock to a noisy and time-varying input laser beam. The PDH scheme presented here is not yet able to handle the situation. How the problem is solved is discussed in Chapter V. 


\section{CHAPTER III}

\section{Experimental apparatus and techniques}

This concentric-cavity experiment is very new in the group. In this chapter, I describe the apparatus and techniques of the experiment, including the vacuum system, excitation lasers, and calibration data. The purpose of this chapter is to provide a detailed technical reference for operating the experiment.

\subsection{In-vacuum near-concentric cavity}

Fig. 3.1(a) shows the experimental geometry of the in-vacuum near-concentric cavity. The cavity is composed of two half-inch concave mirrors with a radius of curvature of $2.5 \mathrm{~cm}$. The cavity is surrounded by six electrodes of $2 \mathrm{~mm}$ diameter, on a circle with diameter of $17 \mathrm{~mm}$. The $1064 \mathrm{~nm}$ lattice laser enters the cavity via the bottom cavity mirror, and is transmitted through the top cavity mirror. A rubidium Magneto-optical trap (MOT) is produced directly at the cavity center.

Fig. 3.1(b) is a photo of the concentric-cavity assembly before it was enclosed inside the external vacuum chamber. The main structural component is a stainless steel cylinder, which shields the cavity mirrors, holds the electrodes in place, and defines the experimental geometry. The large openings in the cylinder are for MOT lasers. The angles of these large openings are oriented such that the six MOT beams overlap the xyz axes of a Cartesian coordinate system with origin at the cavity center. 
There are 10 more small openings for the excitation laser and observation, four of them are on the horizontal plane crossing the cavity center, and the other six are away from the horizontal plane, forming another complete set of optical-access axes aligned to a Cartesian coordinate system with origin also at the cavity center. The two sets of xyz-axis openings are arranged so that all the laser beams passing through the cavity center are either above or below the horizontal plane by $35.26^{\circ}$. The two sets of openings are away from each other by a $60^{\circ}$ rotation about the cavity axis (the rotation axis is in the lab frame).

The cylindrical steel cylinder that holds most components is clamped down on the top of a aluminum base, which is itself screwed to the bottom flange of the external vacuum chamber. The $1064 \mathrm{~nm}$ lattice laser enters the cavity through a channel inside the aluminum base. The first coupling lens, which focuses the lattice laser at

(a)

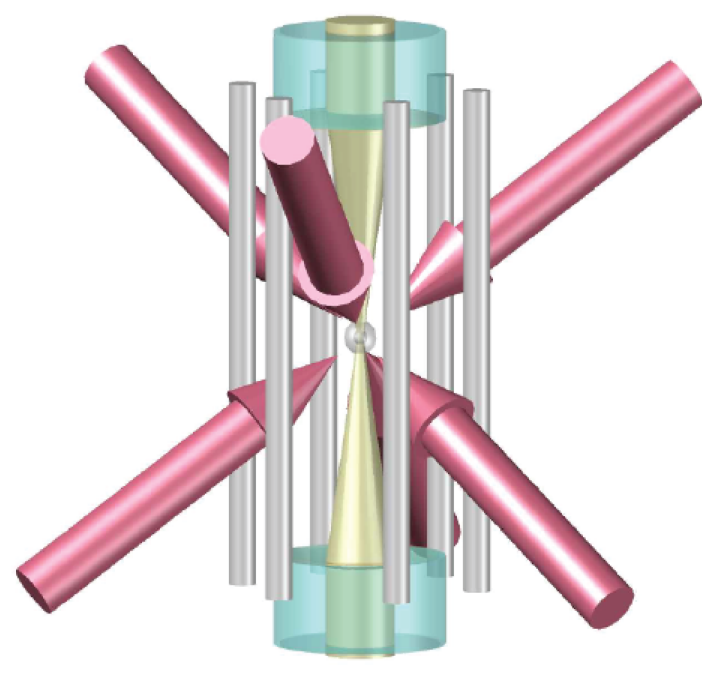

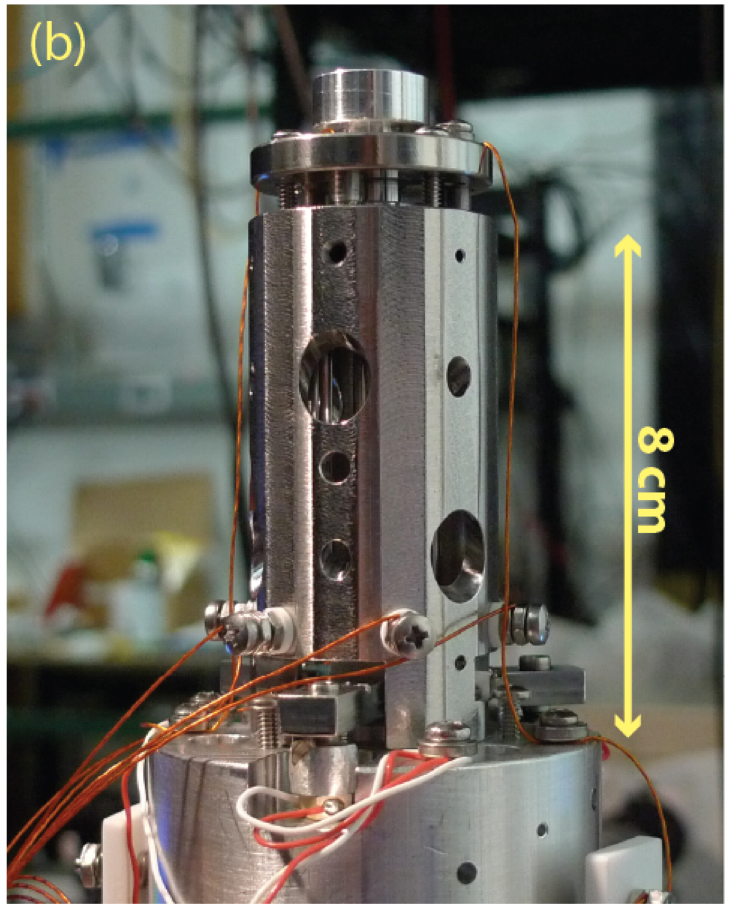

Figure 3.1:

(a) Experimental geometry. The cavity is stabilized to the $1064 \mathrm{~nm}$ lattice laser. A Rb MOT is produced directly at the cavity center, loading the optical lattice trap at the focus where the intensity is the highest. (b) Photo of the concentric-cavity assembly. 

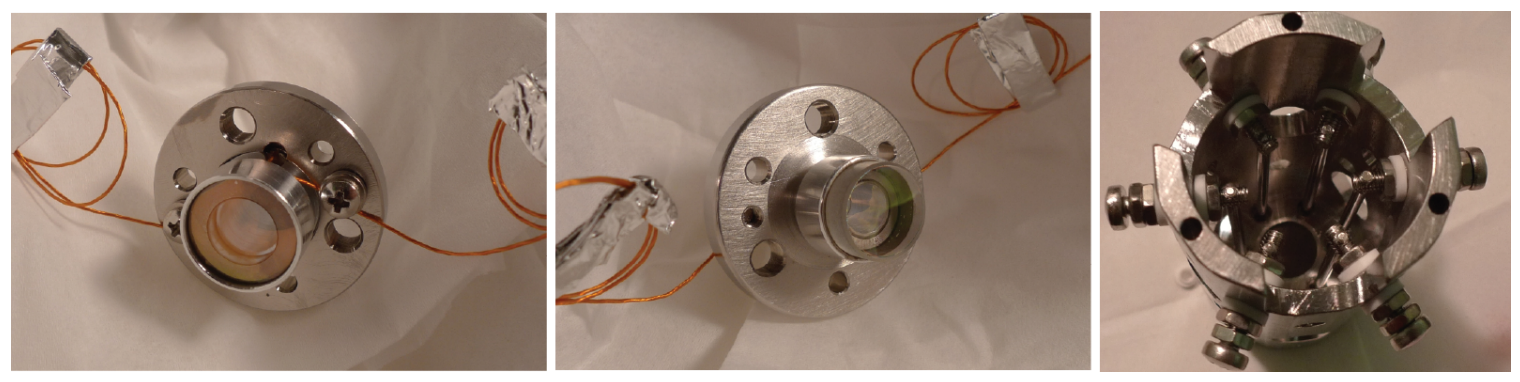

Figure 3.2:

Left: concentric tubes viewed from the coupling lens side. The Kapton wires connect to a ring piezo placed in-between the tubes. Middle: concentric tubes viewed from the side of top cavity mirror. Right: six electrodes inside the stainless steel cylinder. Sec. 3.6 describes the usage of the electrodes.

the cavity center, is glued to the end of the channel inside the base. The second coupling lens, which collimates the transmitted lattice laser, is glued to the top of a set of concentric tubes (see Fig. 3.2). The positions where the lenses are glued to the in-vacuum setup are calibrated by means of Michelson interferometer to ensure that both of the lenses focus at the cavity center.

Both of the cavity mirrors are mounted inside the stainless-steel cylinder with manual angular and translational controls. The top cavity mirror is glued to the set of concentric tubes which supports the coupling lens. A ring piezo is placed inbetween the tubes. The tubes altogether are screwed down to the top of the cylinder by six screws. Three of the screws are $3 / 16^{\prime \prime}-100$ threaded for fine adjustment control. The other three screws are \#4-40 threaded. They lock the tubes in place after the adjustment is done.

The bottom cavity mirror lies on a tripod. The tripod is mounted to the aluminum base in a manner that mimics a conventional kinematic mirror mount (see Fig. 3.3). Each leg of the tripod is fastened down by a spring, while a $3 / 16^{\prime \prime}-100$ screw next to the spring counter-acts the spring force. The screws sit on three plate piezos glued on the aluminum base. These piezos have hand-made adaptors glued on top for accepting the ball tip of the fine-threaded screws. The hand-made adaptors are all different, 


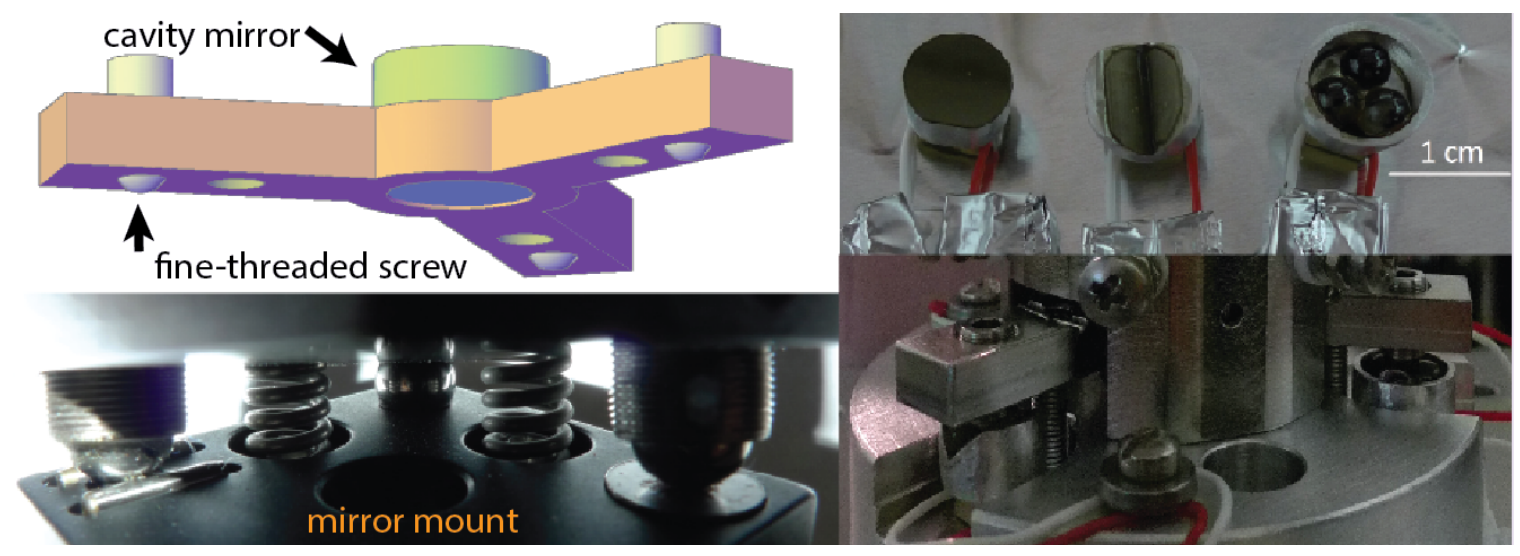

Figure 3.3:

Left: CAD drawing of the tripod and photo of a conventional mirrormount. Right: hand-made adaptor for the three-point contact scheme. Close look of the tripod. The red/white wire pairs connect to the plate piezos.

one with a flat surface, one with a V-shaped groove, and one with three sapphire balls. The adaptors center the tripod to the cavity axis.

Although we cannot access these manual controls after the vacuum chamber is closed, these controls are critical during the assembling process. The lattice modematching and the size of beam waist is optimized with these manual controls. After assembling inside the UHV chamber, the fine tuning of the cavity length and the angular adjustment outside the vacuum chamber are done by voltage control of the piezos in the system. The ring piezo translates the top cavity mirror, and the three plate piezos below the tripod control the angular movement of the bottom cavity mirror.

\subsection{Vacuum system}

The cavity is enclosed inside a one-foot tall cylindrical vacuum chamber. The bottom flange of the chamber has a small viewport for the lattice laser entering the cavity. The top of the chamber is sealed with a large viewport for the convenience of observation (see Fig. 3.4). There are several feedthroughs and viewports attached 


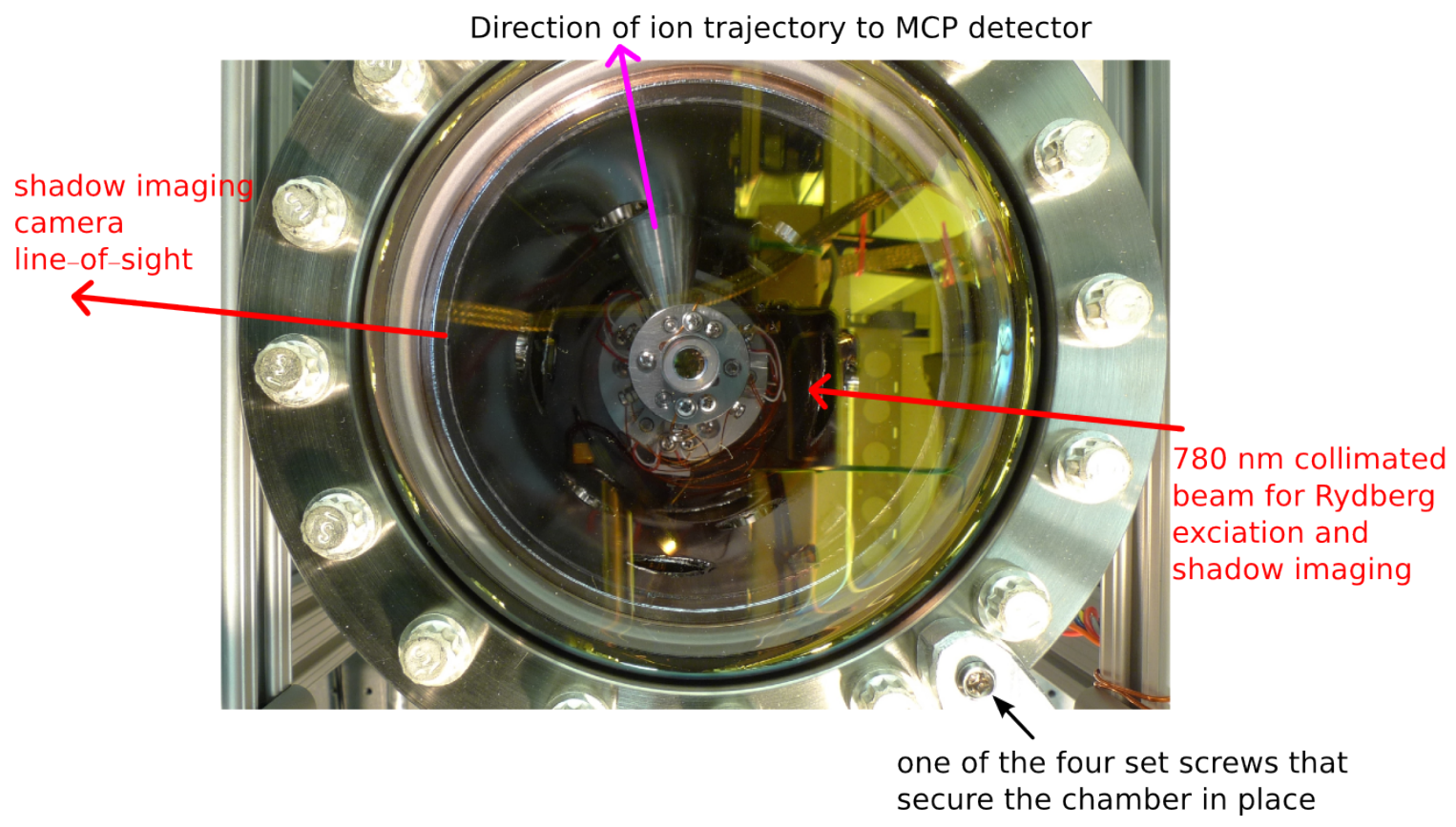

Figure 3.4:

Cavity inside the vacuum chamber seen from the large viewport on the top of the vacuum chamber. The aluminum cone next to the cavity is an ion guard tube, which shields the field-ionized ions on their way to the microchannel plate (MCP) in spectroscopy experiments.

to the side of the chamber. The viewports on the side match the openings in the in-vacuum stainless steel cylinder that holds most cavity components. The other feedthroughs are for electrical connections, vacuum pumps, and a rubidium reservoir. All of the connections are sealed by ConFlat flanges.

A $\mathrm{Rb}$ reservoir is attached to the chamber via an angle valve with a position higher than all of the MOT beam viewports. The $\mathrm{Rb}$ reservoir is warmed up by a heat tape when more $\mathrm{Rb}$ pressure is needed. The $\mathrm{Rb}$ vapor diffuses into the chamber, and is collected by the MOT beams directly at the cavity center. The details of the MOT are discussed in Sec. 3.5 ,

The pressure inside the chamber is in the range $10^{-8}$ to $10^{-9}$ Torr, which satisfies the requirement for a MOT experiment. The chamber was connected to both an ion pump and a turbo pump during the "bake-out" process. Now the valve that was connected to the turbo pump is kept closed, and the turbo pump is removed. The 


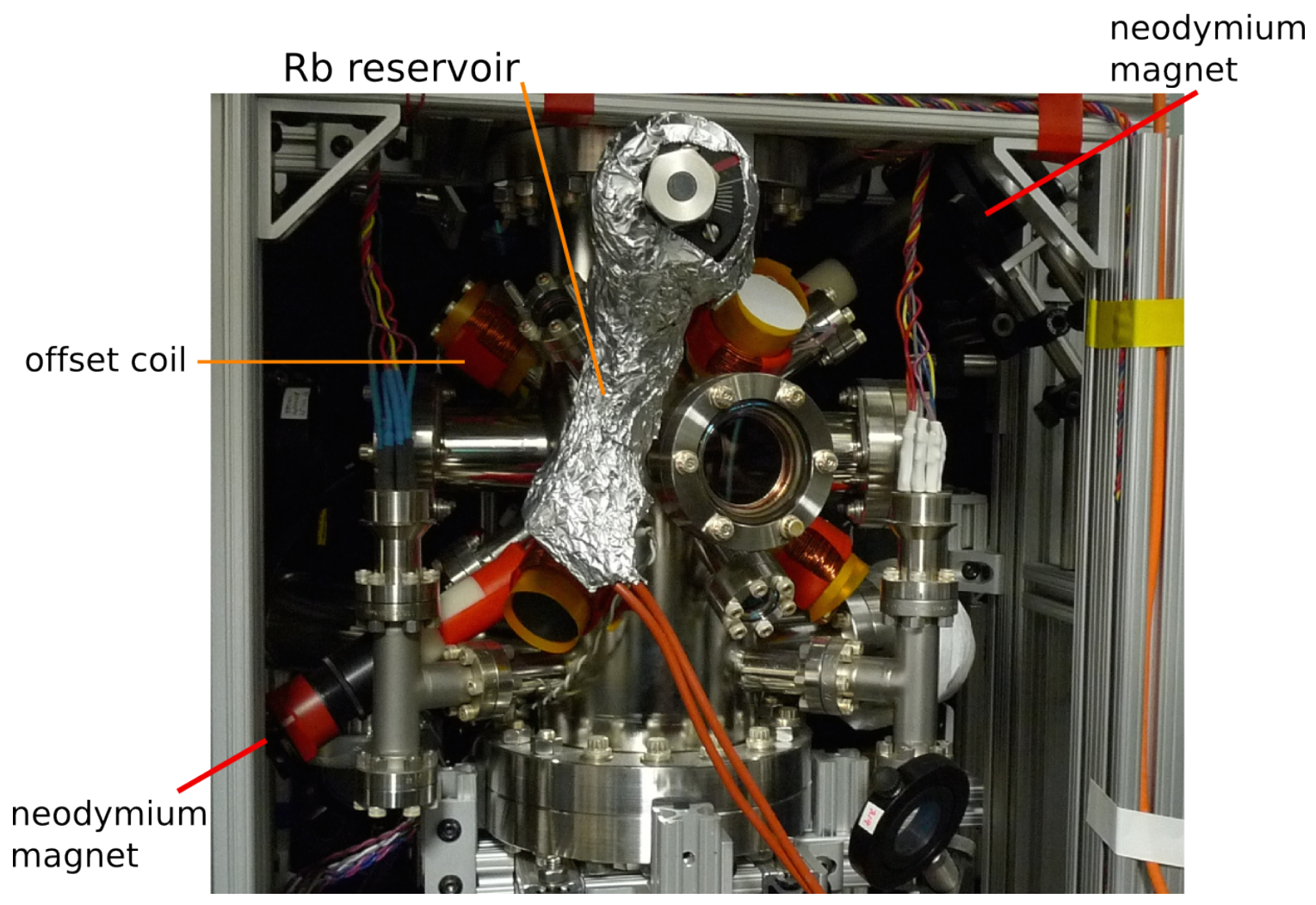

Figure 3.5:

Vacuum chamber front view. The neodymium magnets provide the quadruple magnetic field required for the MOT. The three pairs of offset coils fine tune the MOT magnetic field. The neodymium magnets are 3/4-inch in diameter and 2 inches long. They are wrapped with a piece of paper and fixed inside a 1-inch plastic tube adaptor by frictional force.

pressure inside the chamber is maintained by the ion pump.

\subsection{Excitation lasers}

\subsubsection{Amplified DBR laser system}

The experiment has two amplified $780 \mathrm{~nm}$ Distributed Bragg Reflector (DBR) laser systems. One of them provides the power needed for the MOT cycling transition. The MOT repumping transition is provided by a home-built $780 \mathrm{~nm}$ external cavity diode laser (ECDL) [29]. The other amplified DBR laser system provides the power for $5 \mathrm{~S} \rightarrow 5 \mathrm{P}$ transition in the two-photon excitation and absorption imaging. The original 
design of the amplified DBR laser system is by Erik Power, with modification advice from Rachel Sapiro.

In the ECDL scheme, the wavelength of the laser is tuned by using a reflecting grating to direct the Littrow reflection back to the laser diode. The angle of the grating is controlled by fine-threaded screws, and is fine-tuned by a plate piezo attached to the back side of the grating. With the Littrow feedback, we can force the laser diode to operate at a wavelength that is a few nm away from the central wavelength of the laser diode. The wavelength tuning of the DBR laser diode is somewhat different. The laser diode has a stack of distributed Bragg reflector fabricated on-chip next to the laser gain medium. The reflector enables wavelength-dependent feedback; there are no mechanical components in a DBR laser. The DBR laser output frequency can be current modulated at high speed, and the current response is very linear.

For all the newly-built DBR laser systems in the lab, the laser diodes are from Photodigm, Inc. The laser diode is packed inside a TO-8 package. A thermo sensor and a Peltier thermoelectric cooler are also packed inside the TO-8 can, which is very convenient. The linewidth of the laser is about $1 \mathrm{MHz}$, and the emission wavelength is tunable over several $\mathrm{GHz}$ by fast current modulation without mode hops. The long-term stability of DBR lasers is also much better than the ECDLs. The only disadvantage of the DBR lasers so far is their cost, which is considerably higher than ECDLs.

The amplified DBR laser system is built inside a $4^{\prime \prime} \times 5^{\prime \prime} \times 15^{\prime \prime}$ aluminum box. Fig. 3.6 shows the top view of the components inside the box (from the one that provides the $5 \mathrm{~S} \rightarrow 5 \mathrm{P}$ transition laser). The DBR laser diode is mounted on a black housing. The coupling lens for the laser emission is mounted to the black housing with translational controls in both $\mathrm{x}$ - and y-axes. The collimated output of the DBR laser diode first passes two Faraday isolators. A small portion of the output is sampled for a saturated absorption spectroscopy sat. spec. and a temperature-controlled 
Fabry-Pérot interferometer (FPI). The majority of the laser power is directed to a Tapered Amplifier (TA) laser. The coupling is maximized by the adjustment of the two mirrors at the corners in the box. The TA laser is mounted inside a water-cooled copper block assembly. The water-cooling loop is driven by an aquarium pump. The copper blocks also hold a pair of coupling lenses: one focuses the DBR laser seeding at the TA gain medium, and the other collimates the amplified output from the gain medium. The laser output from a TA usually has a significant astigmatism. To correct the beam profile, the output of the TA is collimated by a cylindrical lens and the size of the beam is adjusted by a telescope.

Fig. 3.7 shows the Fabry-Pérot interferometer transmission spectrum and saturated absorption spectrum (for details see Sec. 3.4) of the amplified DBR laser system for the $5 \mathrm{~S} \rightarrow 5 \mathrm{P}$ transition. In Fig. 3.7, the DBR laser diode is current-modulated, and the output is sampled for both Fabry-Pérot interferometer and sat. spec. at the same time. The saturated absorption spectrum provides absolute frequency reference for the FPI transmission spectrum in this case, since the transition frequencies for the ${ }^{85} \mathrm{Rb}$ cycling and repumping transitions are well-known. The difference in frequency between the $\mathrm{F}=3 \rightarrow 4$ and $\mathrm{F}=2 \rightarrow 3$ transitions is 2915.092(68) $\mathrm{MHz}$ [4]. The

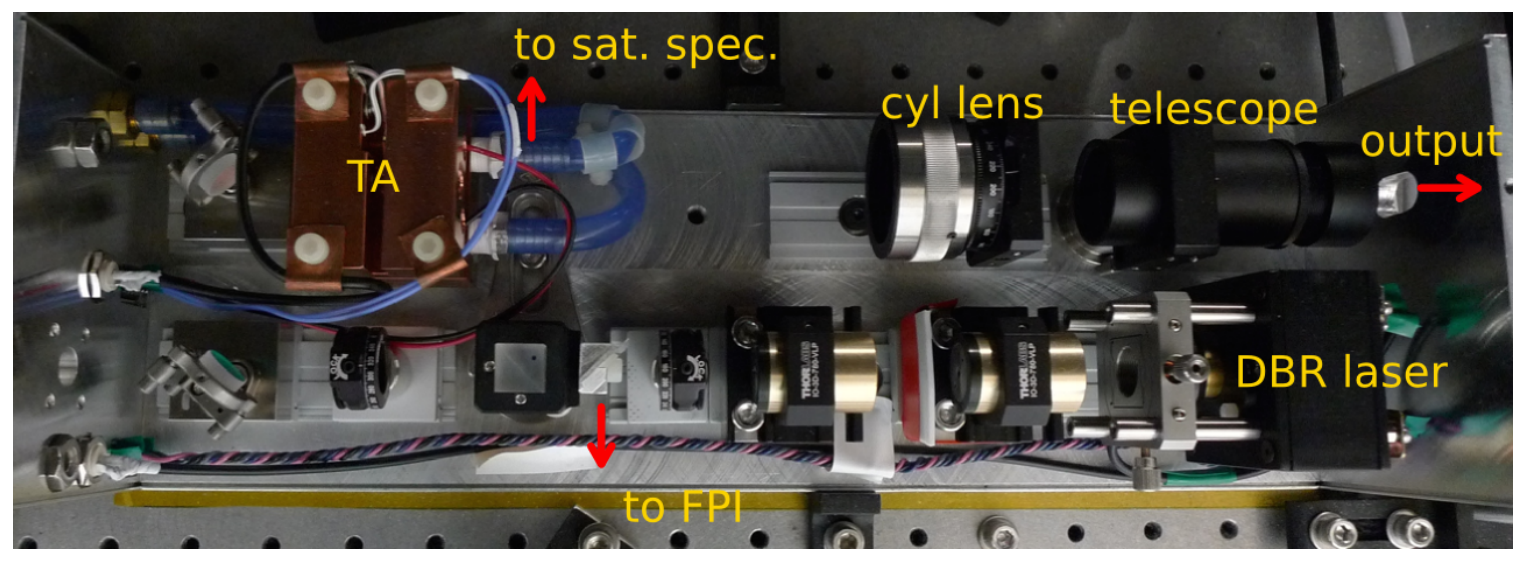

Figure 3.6:

Amplified DBR laser system. The components are all mounted on fixedheight, customized pedestals. Some of them can freely slide on the bottom aluminum rails. 
(a)
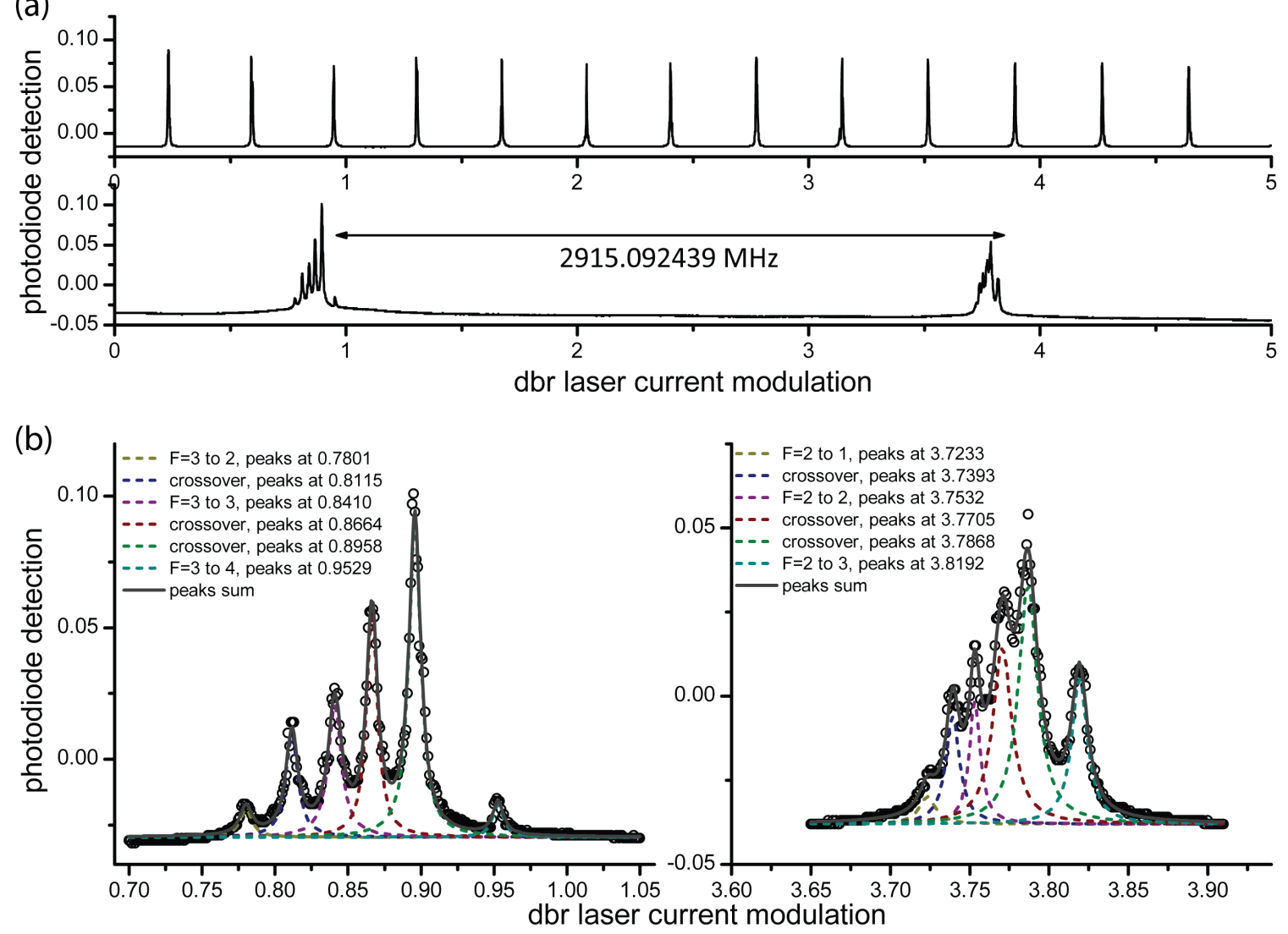

Figure 3.7:

(a) Fabry-Pérot interferometer transmission spectrum (top) and saturated absorption spectrum for rubidium-85 (bottom). The DBR laser diode is current modulated, and its output is sampled for both spectra at the same time. (b) A zoom-in of the saturated absorption spectra. The absorption peaks are fitted with Lorentz amplitude function. The validity of Lorentz peak-findings is examined by comparing the centers of the crossover peaks [1] to their expected position, which is in the middle of two real peaks. The difference is within $0.1 \%$. The centers of the $\mathrm{F}=3 \rightarrow 4$ and $\mathrm{F}=2 \rightarrow 3$ transition gives the frequency reference $2915.092439 \mathrm{MHz}$.

centers of the absorption peaks are determined using a multiple-Lorentzian fit,

$$
y=y_{0}+\frac{2 A}{\pi} \frac{w}{4\left(x-x_{c}\right)^{2}+w^{2}}
$$

where $x_{c}$ is the parameter for the peak center. The output wavelength of the DBR laser is nearly linear under current modulation over several $\mathrm{GHz}$, as can be seen by the equal spacings between the FPI transmission peaks in Fig. 3.7(a). 
The FPI consist of a confocal cavity with cavity length of $20 \mathrm{~cm}$. The FSR is $374.682 \mathrm{MHz}$ (standard error of the mean $0.170 \mathrm{MHz}$ ), determined by repeating the spectroscopic measurement as shown in Fig. 3.7. The cavity is air-sealed inside a kwik-flange nipple, wrapped with heat tape, and then covered by $1^{\prime \prime}$ thick pink foam to prevent thermal drift caused by the changing room temperature. The power dissipated by the heat tape is driven by a temperature controller. The positions of transmission peaks relative to the saturated absorption signals can be moved by changing the temperature setting of the FPI. The transmission peaks move by half of a FSR when the temperature is changed by $2{ }^{\circ} \mathrm{C}$.

For most of the rubidium MOT experiments, the saturated absorption spectrum also functions as a source of error signal for an electronic feedback circuit. The feedback circuits stabilize the laser frequency to the zero-crossing of the slopes of the spectral peaks. For later experiment with large lattice light shift, large $5 \mathrm{~S} \rightarrow 5 \mathrm{P}$ transition detuning is required. The transmission spectra of the temperature-controlled FPI provide extra spectral peaks in spectral region where no atomic transition lines exist. This ability is critical for a large part of the work presented in this thesis.

\subsubsection{Frequency-doubled blue laser}

The output from a $960 \mathrm{~nm}$ ECDL is increased by a TA laser, and then frequencydoubled by a frequency-doubling stage which yields the $480 \mathrm{~nm}$ upper transition laser $(5 \mathrm{P} \rightarrow$ Rydberg state). The design of the TA laser was made by Luís Felipe Gonçalves.

Fig. 3.8 shows the photos of the TA laser. The center piece is made of copper. The C-mount TA laser chip is screwed to the M2-threaded hole at the center of the copper piece. The copper piece is dropped into the bottom brass plate, and clamped downward in place tightly by the large brass piece.

The coupling lenses for the TA are mounted into aluminum tubes with fineexternal threads, which match the inner threads of the two cuboid brass lens mounts. 
The aluminum tube can freely move in or out relative to the brass cuboids during the laser-collimation process, and then be locked stationary by a set screw after the adjustment is done. The brass cuboids are attached to the large brass piece by four M3 screws with split lock washers. The position of the coupling lenses can be translated in both horizontal and vertical directions by balancing the pressure exerted by the 6-80 fine-threaded screws.

\subsubsection{Pressure-tuned Fabry-Pérot interferometer}

A few percent of the $960 \mathrm{~nm}$ ECDL output is directed to a wavelength meter and a pressure-tuned Fabry-Pérot interferometer. Details about the FPI are presented in refenrence 30. The FPI is made of a $15 \mathrm{~cm}$ long confocal cavity. The confocal cavity is sealed inside a kwik-flange tubing, filled with high-index-of-refraction, inert gas $\mathrm{SF}_{6}$. A bellow is attached to the kwik tubing, and the bellow is pulled/pushed by a step motor driving a optical translational stage. The stepping of the step motor changes the volume of the bellow, which in turn changes the pressure and refractive index inside the entire kwik tubing. The tiny change of FSR due to the change of refractive index shifts the position of the peaks on the transmission spectrum. As a result, the transmission peaks translates in steps as the step motor moves.

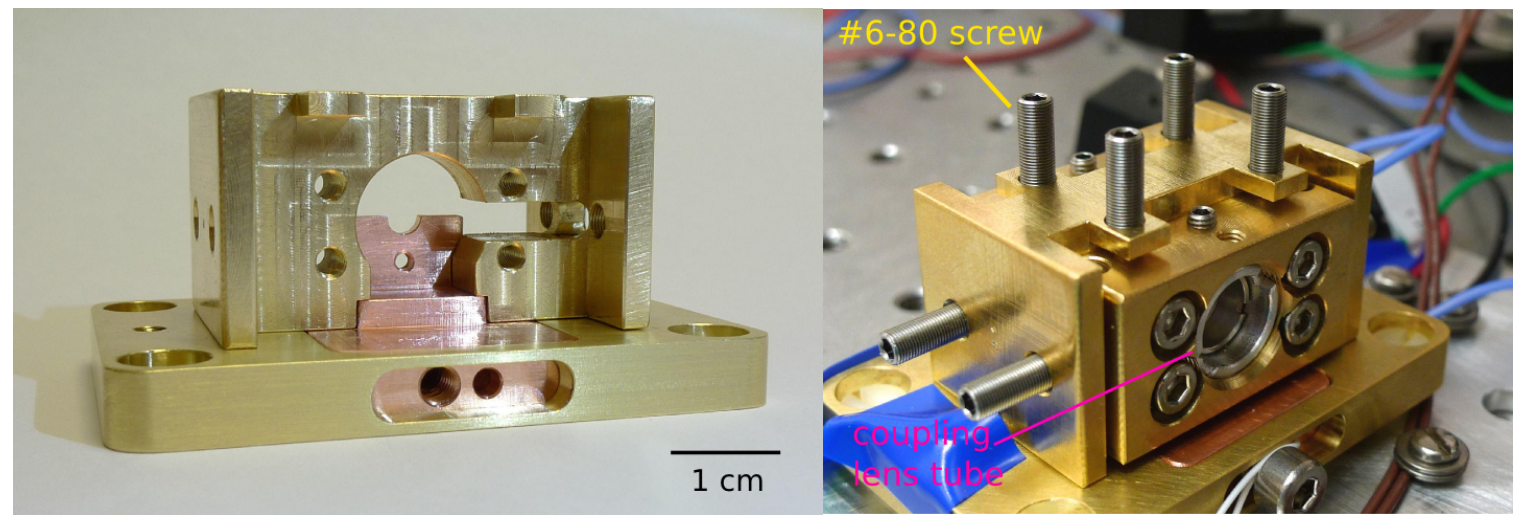

Figure 3.8:

Left: center pieces of the TA amplifier laser. Right: TA amplifier laser assembly. 
During a two-photon excitation experiment, the lower transition, $780 \mathrm{~nm}$ laser is locked to a $\mathrm{Rb}$ atomic transition, and the upper transition $480 \mathrm{~nm}$ laser, frequency doubled from the $960 \mathrm{~nm}$ laser, is scanning across the Rydberg-atom excitation resonance. This is done by stabilizing the $960 \mathrm{~nm}$ ECDL to the nearest FPI peak, and programming the step motor to step toward the resonance. The PID feedback circuit, which stabilizes the $960 \mathrm{~nm}$ ECDL, is able to lock the laser frequency to the same peak and allow the step motor scanning over a range of about $750 \mathrm{MHz}$ at $960 \mathrm{~nm}$ (1500 MHz at $480 \mathrm{~nm})$.

The movement of the step motor is recorded in step number in the raw data. Fig. 3.9 shows the calibration factor of step number into $\mathrm{MHz}$ at $480 \mathrm{~nm}$. Calibration is obtained with $69 \mathrm{~S}$ two-photon excitation spectra. The upper transition, frequency doubled blue laser, is switched on and off by a single-pass acousto-optic modulator $(\underline{\mathrm{AOM}})$. The AOM generates multiple orders of deflected blue light. The frequency difference between consecutive orders is the same as the frequency of the radio frequency source driving the AOM, which is $361 \mathrm{MHz}$. As a result, the difference in step number between two 69S lines which use consecutive deflection orders of the $\mathrm{AOM}(-1$ and -2 order) equals $361 \mathrm{MHz}$.

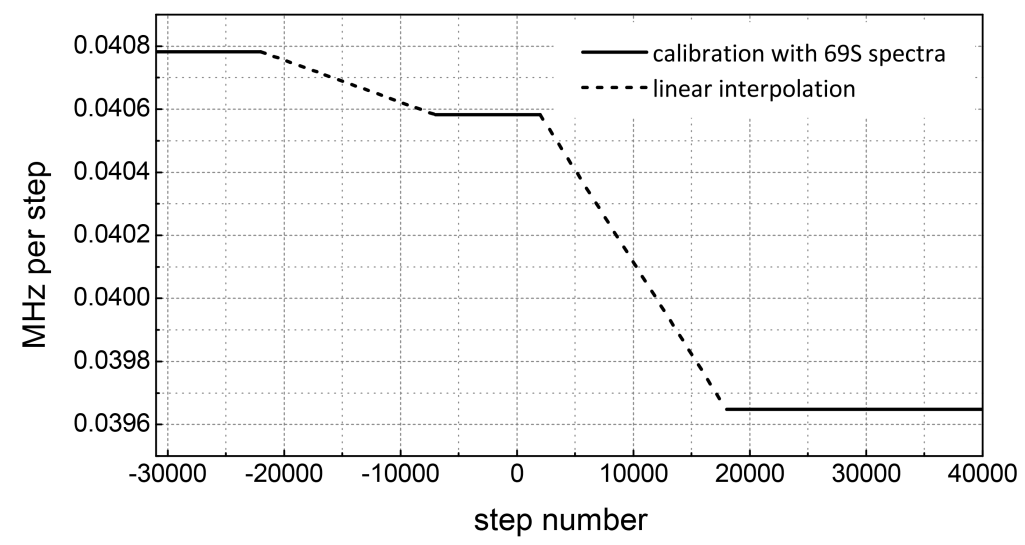

Figure 3.9: Calibration factor of the step motor (step number to $\mathrm{MHz}$ at $480 \mathrm{~nm}$ ). The slight change in calibration is mostly due to the non-linear response of the bellow. 
In later experiments involving large lattice light shifts, the spectroscopic searching range is usually several $\mathrm{GHz}$, which is beyond the PID feedback circuit's tracking range. The spectroscopy in this condition requires manually turning the $960 \mathrm{~nm}$ grating voltage offset control and counting the transmission peaks being swept during the manual search. The final frequency shift relative to the starting point of the twophoton excitation spectra will then be the frequency shift converted from step number, plus the number of peaks passed times the FSR of the FPI at $480 \mathrm{~nm}$.

The calibration of the FSR of the $960 \mathrm{~nm}$ FPI is done by referencing the distance of main Gaussian cavity mode to the side bands generated by a single-pass AOM (see Fig. 3.10 on the cavity transmission spectra. The collimated output of the $960 \mathrm{~nm}$ laser focuses at the crystal inside the AOM, and then the AOM output is collimated before being sent to the FPI. When a focused beam is deflected by the crystal, it is very easy to get higher order modes creeping in the direction of the zero order mode, and vice versa. The leakage of the higher orders appears as side bands next to the main cavity mode. The separation between the side bands and major peaks equals the frequency shift by the AOM, which is the same as the RF driving frequency of

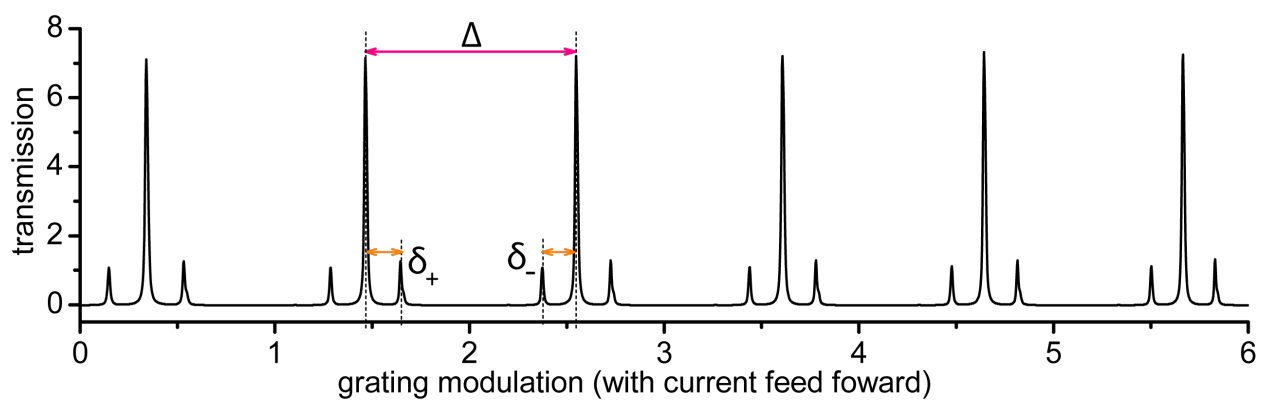

Figure 3.10: 960 nm FPI transmission spectra. The side bands are caused by higherorder deflections of a single-pass AOM. Note the response of the grating modulation is not as linear as the current response of a DBR laser. The ratio of the FSR to sidedands is used in the calculation to compensate this nonlinearality. 
the AOM $(80 \mathrm{MHz})$. The FSR is calculated as,

$$
\mathrm{FSR}=\frac{\Delta}{\delta_{+}+\delta_{-}} \times 2 \times 80 \mathrm{MHz}
$$

where the values of $\Delta, \delta_{+}$and $\delta_{-}$are measured as shown in Fig. 3.10. The averaged FSR is $489.37 \mathrm{MHz}$ at $960 \mathrm{~nm}$, with standard error of the mean 0.56 MHz. The FSR at $480 \mathrm{~nm}$ is twice the number of the FSR at $960 \mathrm{~nm}$.

\subsubsection{Computer control of the experiment}

The MOT lasers and both excitation lasers are switched on and off by AOMs. The RF driving power of the AOM is triggered on and off by the Transistor-transistor Logic (TTL) pulses sent out from the computer that controls the timing and dataacquisition of the entire experiment. The computer has an eight-channel counter/timer extension board (National Instruments, NI PCI-6602), a GPIB controller (NI GPIBUSB-HS), an analog input card (NI USB-6003), and an analog output card (Measurement Computing, USB-3114). The computer programs that run these extension cards are developed in LabView (version 12). My experiment inherited LabView programs from the TIP experiment in the group, codes mostly written by Andrew Schwarzkopf.

\subsection{Saturated absorption spectroscopy}

The laser cooling experiments start with cooling and collecting gaseous atoms by laser beams whose frequencies are tuned close to atomic transitions. For a rubidium experiment, the MOT cooling utilizes the $5 \mathrm{~S}_{1 / 2} \rightarrow 5 \mathrm{P}_{3 / 2}$ atomic transition. The transition is at $780 \mathrm{~nm}$, a wavelength which is easily available from semi-conductor diode lasers.

The stabilization of laser frequency to atomic transition in the experiment is based on saturated absorption spectroscopy. To obtain a saturated absorption spectrum, 
a pair of $780 \mathrm{~nm}$ probe laser beams are sent through a room-temperature rubidium vapor cell, one of the probe beams is overlapped with a counter-propagating pump beam that has an intensity higher than that of the probe beam. The transmitted laser intensities of both probe beams are monitored. Fig. 3.11 shows the spectra of both probe beams when the laser frequency is modulated over the rubidium atomic transitions. Without a pump beam present, the transmitted spectra have Dopplerbroadened absorption features, which reflect the velocity distribution of the atom sample inside the vapor cell. With a pump beam, the narrow spectral features of the hyperfine levels burn into the wide Doppler background (Lamb dip, see reference [31] for details.). Due to the Doppler shift, atoms moving at different velocities see on-resonance radiation at different laser frequencies, and then broaden the absorption feature. With the strong and counter-propagating pump beam, the small portion of atoms that are stationary is depleted by the pump beam, resulting in a narrow trans-
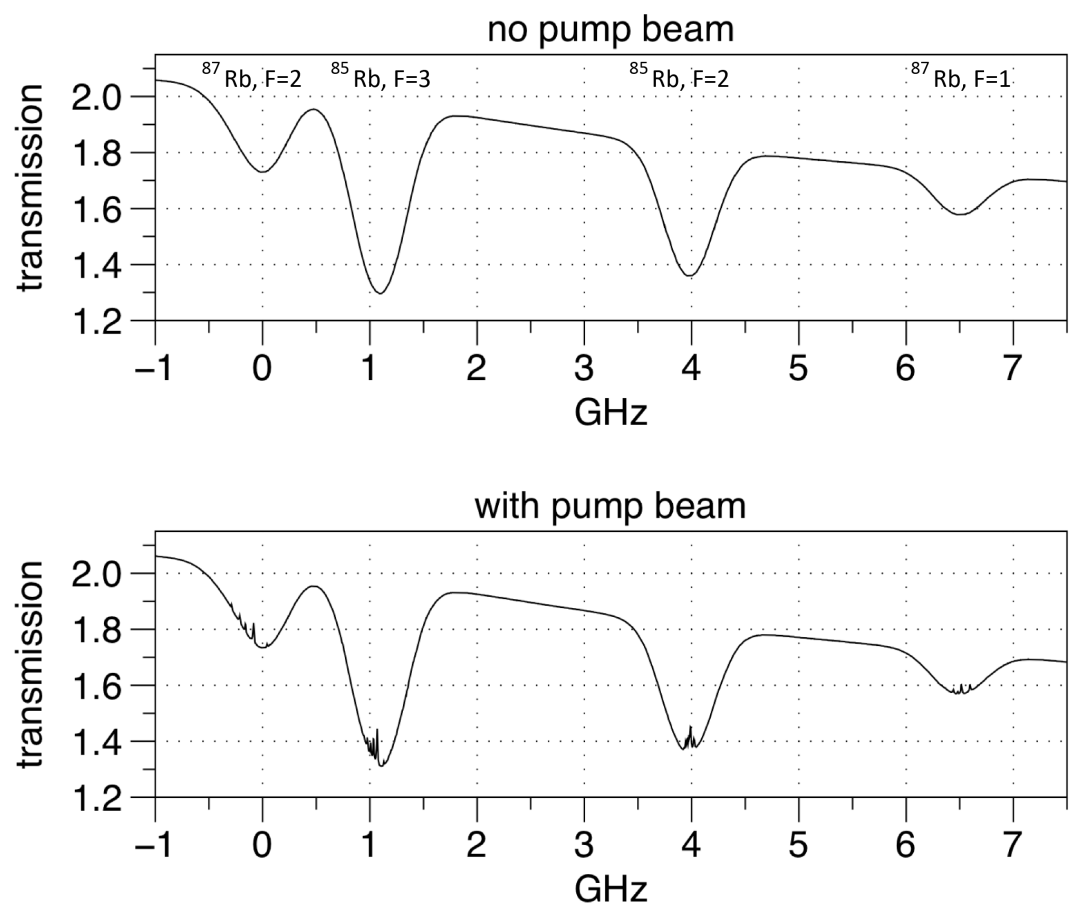

Figure 3.11: Wide scan of absorption spectra of rubidium $5 \mathrm{~S}_{1 / 2} \rightarrow 5 \mathrm{P}_{3 / 2}$ transition. 
parent window for the probe beam. Narrow spectral features at hyperfine transitions thus are revealed on the wide Doppler-broadened absorption feature. By sending both spectra to a differential amplifier, the Doppler background is removed. The resulting signal is shown in Fig. 3.12. The hyperfine spectral lines provide absolute atomic transition frequency reference.

The laser frequency stabilization is done by feeding the saturated absorption spectroscopic signal to an electronic feedback circuit, e.g. PID feedback or a simple integrator. These lock circuits stabilize laser to a zero-crossing of a slope on the spectra. By changing the offset of the spectral signal, the lock circuit can stabilize the laser frequency to virtually both sides of the narrow peaks in the saturated absorption spectra. For a position near the spectral peak where the zero-crossing is hard to acquire, e.g. the ${ }^{85} \mathrm{Rb}\left({ }^{87} \mathrm{Rb}\right)$ MOT cycling transition, a zero-crossing at the desire location can be created by Zeeman shift. This scheme is known as dichroic atomic vapour laser lock, or DAVLL, in the literature. Wrapping the Rb vapor cell with a solenoid, a magnetic field with magnitude of about 10 Gauss shifts the spectral line far enough from the zero-field cycling transition for MOT operation. More control of the zero-crossing position is acquired by "mis-aligning", or by changing the polarization of the pump beam. When the laser is locked, sending the output of the laser to a single-pass or a double-pass AOM, which deflects the laser beam and offsets the laser frequency, provides additional tuning range.

\subsection{Magneto-optical trap}

A very clear introduction to Magneto-optical traps and optical molasses can be found in the reference book Atomic Physics [32] by C. J. Foot. In this section, I focus on the practical aspects of obtaining a MOT in my experiment.

The necessary elements to get a MOT are: (1) six circularly-polarized MOT laser beams along the xyz axes of a Cartesian coordinate system, (2) a quadruple mag- 
netic field with the field minimum at the origin. The MOT magnetic field is usually provided by a pair of coils in anti-Helmholtz configuration. The MOT laser includes two components, one for the cycling transition and one for the repumping transition (see Fig. 3.12 for spectra and level diagram). For a ${ }^{85} \mathrm{Rb}\left({ }^{87} \mathrm{Rb}\right) \mathrm{MOT}$, the cycling transition laser drives the $\mathrm{F}=3(2) \rightarrow \mathrm{F}^{\prime}=4(3)$ transition. However, the atoms can also be off-resonantly excited to the $\mathrm{F}^{\prime}=3(2)$ level by the cycling transition laser. From here, the atoms can decay to the $\mathrm{F}=2(1)$ lower level of the $5 \mathrm{~S}_{1 / 2}$ state. This lower $5 \mathrm{~S}$ level is about $3 \mathrm{GHz}$ away from the cycling transition $(6.8 \mathrm{GHz}$ in the case of ${ }^{87} \mathrm{Rb}$ ), which is so far-detuned that the atoms in the level will not be brought back to the laser cooling cycle by the cycling-transition laser. The repumper, which drives the transition $\mathrm{F}=2 \rightarrow \mathrm{F}^{\prime}=3\left(\mathrm{~F}=1 \rightarrow \mathrm{F}^{\prime}=2\right.$ for $\left.{ }^{87} \mathrm{Rb}\right)$, brings the lost atoms back to the cooling cycle. The laser power of the cycling transition is about $15 \mathrm{~mW} / \mathrm{cm}^{2}$ per MOT beam. For the repumper transition, about $0.5 \mathrm{~mW} / \mathrm{cm}^{2}$ is sufficient, although when the deep optical lattice trap is on, more repumping power is needed. The MOT will essentially not work without the repumper laser.
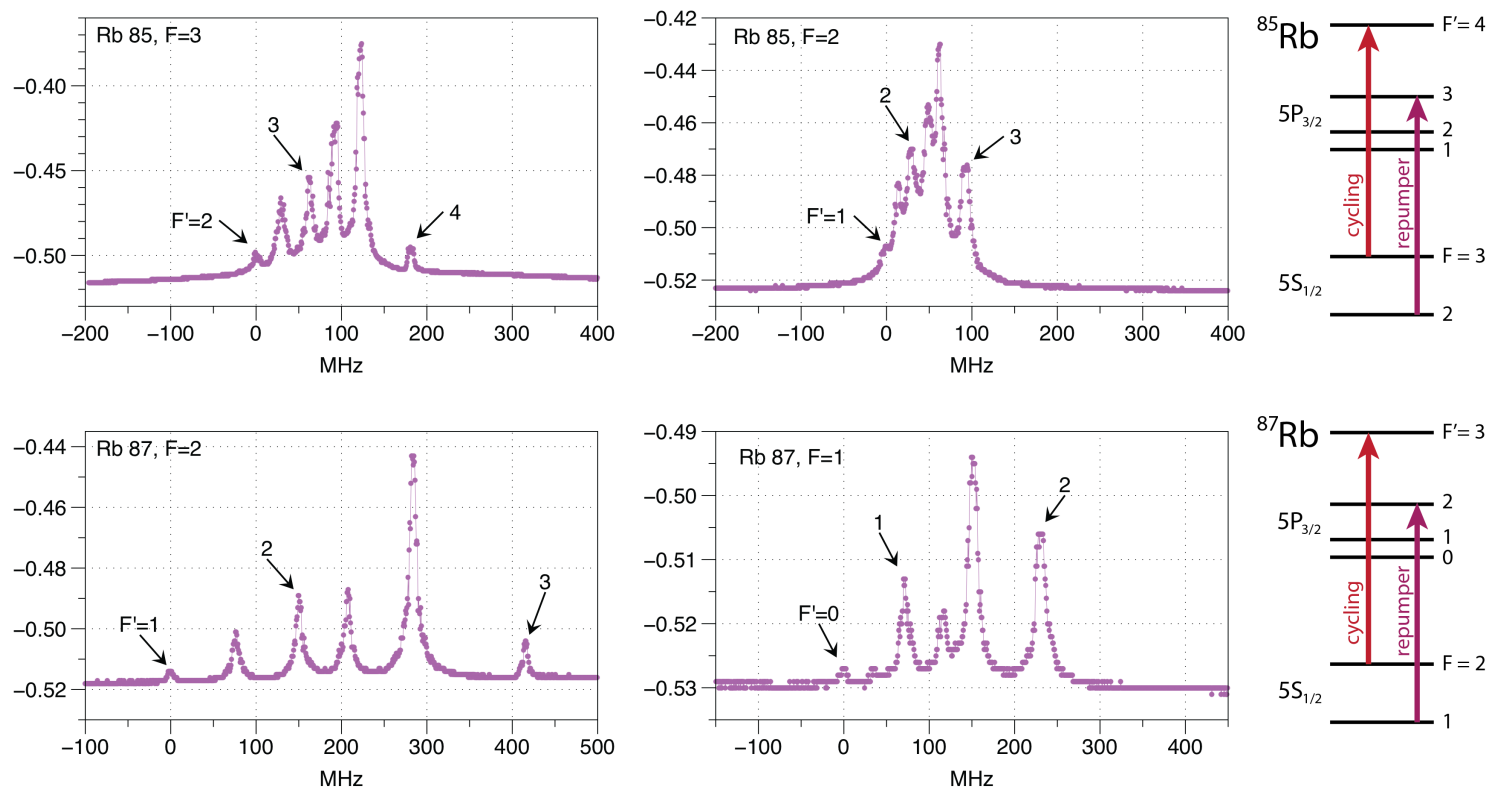

Figure 3.12: Saturated absorption spectra for rubidium 85 and 87. 
A common method for loading atoms into a MOT is by primary-secondary MOT configuration. In my experiment, however, as in numerous other systems, the MOT traps the rubidium atoms directly from the room-temperature rubidium vapor inside the vacuum chamber. Therefore, the total number of atoms trapped by this MOT configuration is considerably lower than the primary-secondary MOT scheme. Also, due to the size of the openings in the stainless steel cylinder which shields the cavity, the MOT beam size can only be made as large as $8 \mathrm{~mm}$, which further reduces the ability to collect atoms. Another difference in this experiment is that due to limited space anti-Helmholtz coils were found to be less practical than a quadruple MOT magnetic field provided by a pair of rod neodymium magnets (Fig. 3.5). The magnetic field gradient at the mid point of the pair of magnets is about 19.5 Gauss $/ \mathrm{cm}$. This pair of rod neodymium magnets can only mounted in a manner such that the magnetic field experienced by the atoms is actually about $15^{\circ}$ off from the orientation with the maximal field. (see Fig. 3.5). Nevertheless, despite of the deviations from a common setup, the MOT traps atoms and loads the optical lattice. My experiment has a rapid experimental repetition rate because of this MOT loading scheme: the MOT collects atoms directly at the cavity center and no atom transfer is needed.

The deep optical lattice experiment uses either ${ }^{85} \mathrm{Rb} \mathrm{MOT}$ or ${ }^{87} \mathrm{Rb} \mathrm{MOT}$. The switch between different isotopes only requires us to lock the lasers to different saturated absorption spectral lines. Generally, the ${ }^{85} \mathrm{Rb} \mathrm{MOT}$ is brighter than the ${ }^{87} \mathrm{Rb}$ MOT, because the ${ }^{85} \mathrm{Rb}$ isotope is about three times more abundant than ${ }^{87} \mathrm{Rb}$. However, the $5 \mathrm{~S} \rightarrow 5 \mathrm{P}$ excitation scheme of the ${ }^{87} \mathrm{Rb}$ MOT in spectroscopy studies is less complicated, mostly due to the larger hyperfine splitting of the $5 \mathrm{~S}$ hyperfine levels, and fewer light-shifted lines in the $5 \mathrm{P}_{3 / 2}$ level. 


\subsection{Stark spectroscopy}

The detection of Rydberg atoms is achieved by field-ionization with the electrodes surrounding the cavity, and ion-detection with a microchannel plate (MCP) detector [33. Fig. 3.13 shows the relative position of the MCP and the electrodes to the atom-trapping region.

The field strength required to ionize the Rydberg atoms is not extremely high; usually several tens of $\mathrm{V} / \mathrm{cm}$ is sufficient. The ionization field launches the positive ions toward the MCP, which is a few inches downstream. The MCP detector is composed of two micro channel plates in the Chevron configuration, plus a phosphor screen attached to the Chevron. The front plate of the MCP detector, with a voltage of $-1015 \mathrm{~V}$, acts like a huge sink for the positive rubidium ions. The second plate of the MCP and the phosphor screen are at $+735 \mathrm{~V}$ and $+3375 \mathrm{~V}$, respectively. When a positive ion reaches the MCP front plate, it triggers a chain reaction at the spot, releasing many electrons which then generate a blip at the MCP phosphor screen. Thus, the blips on the phosphor screen reflect the spatial distribution of Rydberg atoms before field ionization. When the amplified electron outputs reach the phosphor screen, they create a voltage spike on the DC high-voltage background. To count the number of detected ions, a high-pass filter is inserted between the DC high-voltage supply and the phosphor screen. The pulses are filtered out by the high-pass filter, and then sent to a photon counter. The photon counter converts the electrical pulses into a number of detected events. The detected counts are plotted as a function of the step-motor step number, and then converted into a count rate vs. frequency plot, using the calibration factors in Fig. 3.9.

Rydberg atoms are known to be very sensitive to electric fields. A small stray electric field can cause significant spectral line shift and line splitting, especially for high-lying Rydberg states. The unknown stray electric field is compensated by Stark spectroscopy [5] of Rydberg D lines. The spectroscopy is done by taking D line spectra 

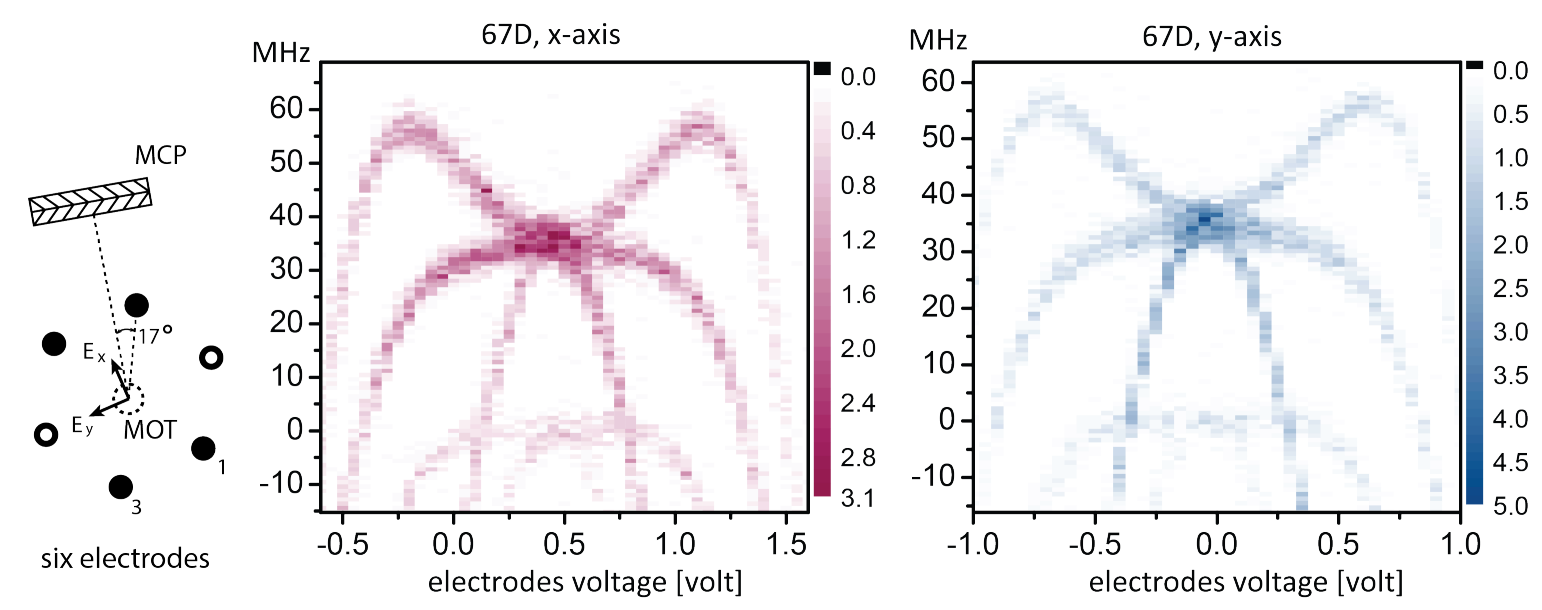

Figure 3.13:

Stark maps for the rubidium 67D level. The electric field in $\mathrm{x}$ direction is controlled by four of the electrodes surrounding the cavity. The four electrodes are marked by solid disks. The electric field in y direction is controlled by the other two electrodes, marked by open circles. The stray electric field is compensated at the voltage setting where the lines have no splitting. The maximal upper-shift in frequency of $67 \mathrm{D}_{5 / 2}$ lines happens at $-0.21 \mathrm{~V}$ and $1.12 \mathrm{~V}$ of $\mathrm{x}$-axis scan, and $-0.70 \mathrm{~V}$ and $0.62 \mathrm{~V}$ of y-axis scan. These maxima on the map give a rough calibration of electric field.

at different voltage settings of the six electrodes during the Rydberg excitation phase. The spectra are plotted against the electrode voltage setting. See Fig. 3.13 for an example. The specific voltage setting that shows no splitting on the plot would be the best voltage setting, since it best compensates the stray electric field.

The voltage settings of the electrodes are independent from each other. The voltages are assigned such that the six electrodes provide the control of electric field in the $\mathrm{x}$ - and $\mathrm{y}$-direction. The electric field is not compensated along the $\mathrm{z}$-axis due to the geometry of the electrodes, but we do not observe a line splitting due to the lack of control along this direction.

During the field ionization phase, the high voltage is assigned to the two electrodes which are farthest from the MCP. The high voltage is assigned with a ratio of about 1:3 to launch the ions toward the MCP (see Fig. 3.13). With a voltage setting of $200 \mathrm{~V}$ and $71 \mathrm{~V}$ applied to these two electrodes, the electric field is numerically calculated 
to be about $73 \mathrm{~V} / \mathrm{cm}$ at the trapping center. The two electrodes that are nearest to the MCP are assigned with small offset voltage to optimize the direction of ions trajectory.

\subsection{Optical dipole traps and optical lattices}

Optical dipole traps [34] use focused laser beams to trap atoms. The atoms are attracted to, or repelled from the high intensity region, depending on the direction of frequency detuning of the laser beam relative to the atomic transition and atomic polarizability values. If the laser frequency is red-detuned, then the atoms are attracted to the region of high intensity. The atoms are attracted to low intensity regions if the laser is blue-detuned.

For a laser beam with intensity $I$ and detuning $\delta$, the trap depth is proportional to $\frac{I}{\delta}$, and the scattering rate is proportional to $\frac{I}{\delta^{2}}$. Experimentally, we usually use a far-detuned focused $1064 \mathrm{~nm}$ laser beam of high intensity (usually on the order of $10^{10} \mathrm{~W} / \mathrm{m}^{2}$ ) to trap rubidium atoms. The large laser detuning ensures that there is not too much heating of the atoms due to photon scattering.

Optical lattices use a similar trapping mechanism. Instead of one single laser beam, optical lattices utilize two or more laser beams to generate standing wave patterns. The spatial confinement of atoms is not only in radial direction relative to the laser beam, but also in the direction of laser propagation due to the periodic standing wave patterns.

The atoms inside the lattice see periodic light shifts due to the AC Stark shift. For

ground state atoms, the $5 \mathrm{~S}$ atomic level is altered by the light field as $-\frac{1}{4} \alpha_{5 \mathrm{~S}} E_{0}^{2}$, where $\alpha_{5 \mathrm{~S}}$ is the dynamic polarizability of the $5 \mathrm{~S}$ level. The polarity of the AC Stark shift depends on the sign of polarizability of the level. For the relevant rubidium levels in this thesis, $\alpha_{5 \mathrm{~S}}$ is positive, $\alpha_{5 \mathrm{P}}$ is negative, and Rydberg states have a negative polarizability in most of the cases. The light shift causes lattice potential wells as 


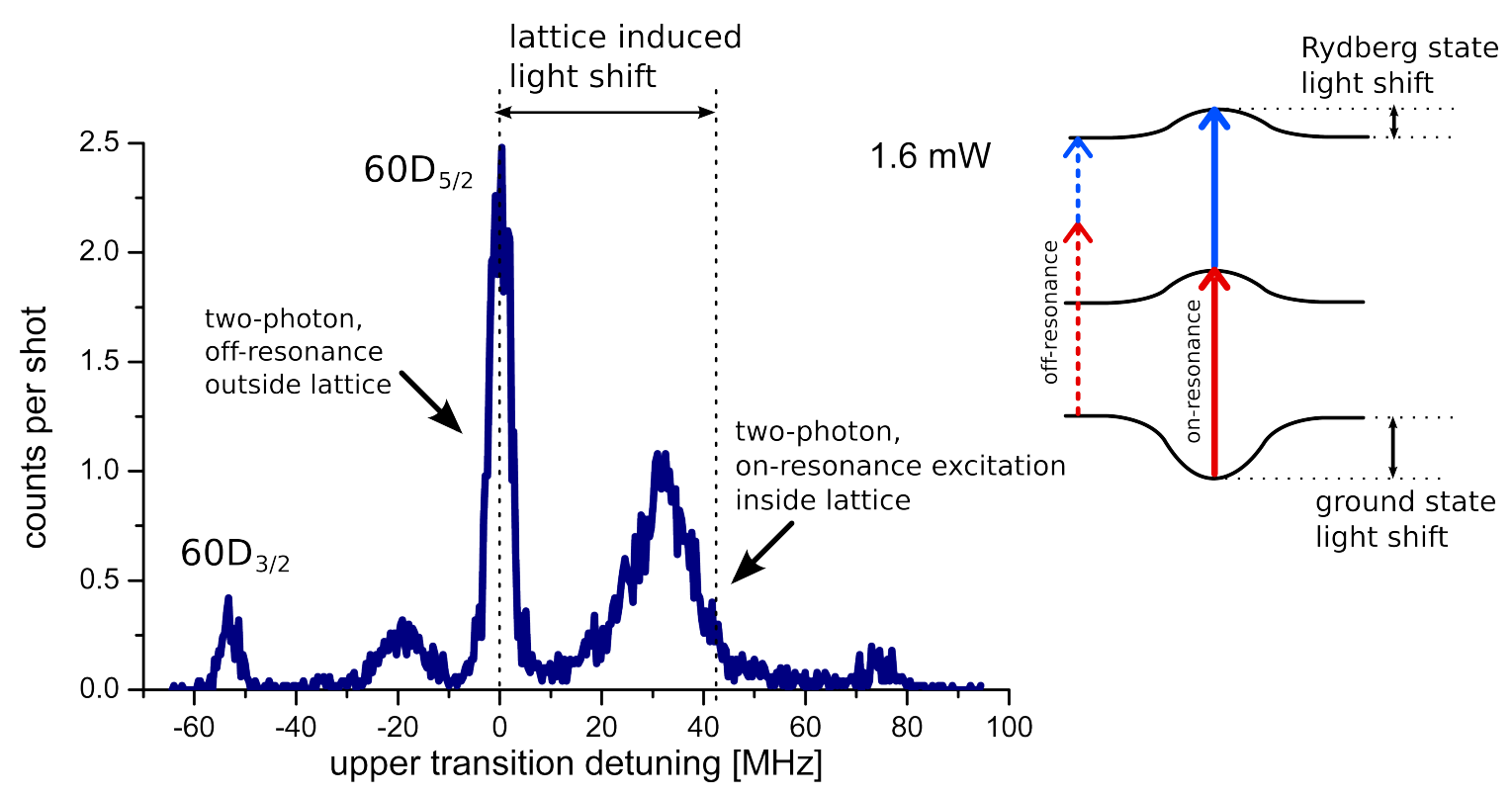

Figure 3.14: two-photon excitation, shallow lattice spectrum.

shown in Fig. 3.14

When taking two-photon excitation spectra of the lattice shift signals, the lower transition laser is frequency up-shifted to compensate the lattice light shift. Fig. 3.14 shows a sample scan in my experiment. The $1064 \mathrm{~nm}$ lattice laser power is conveniently expressed in terms of cavity transmitted power. In this specific scan, the transmitted power is $1.6 \mathrm{~mW}$. The lower transition detuning is up-shifted by $75 \mathrm{MHz}$. The atoms concentrated at the center of the lattice trap are on-resonantly excited, while the atoms outside the lattice are off-resonantly excited. The narrow peaks on the spectra are from the atoms outside the lattice, while the broadened blue-shifted signals are from the atoms inside the lattice. The frequency distance from the center of the narrow line to the blue side of the lattice signal is the maximal lattice-induced light shift, which is a combination of ground-state light shift plus Rydberg state light-shift (see Fig. 3.14). 


\section{CHAPTER IV}

\section{Atom trapping with cavity-generated optical potentials}

In this chapter, I summarize the experiments that have been performed with the cavity-generated optical lattice trapping potentials. The results discussed in this chapter are for shallow lattice potentials, and most of them are published on Phys. Rev. A (http://link.aps.org/doi/10.1103/PhysRevA.89.063409).

\subsection{Introduction}

Fig. 4.1 shows the geometry of cavity-generated optical lattice potentials. The cavity mirrors are centered on the z-axis, so the z-axis is also the lattice axis. At the cavity center, the light shifts depends on both the longitudinal direction (z-axis) and transverse direction ( $\mathrm{x}$ - and $\mathrm{y}$-axes). In the z-axis, the light shift is sinusoidal with a periodicity of $532 \mathrm{~nm}$, due to the standing wave pattern of the $1064 \mathrm{~nm}$ lattice laser. In both the $\mathrm{x}$ - and $\mathrm{y}$-axes, the light shift has Gaussian profile, due to the small waist of the cavity modes; the $\mathrm{e}^{-2}$ waist of the intensity of the $\mathrm{HG}_{00}$ mode is $23 \mu \mathrm{m}$ in both the $\mathrm{x}$ - and $\mathrm{y}$-axes.

This cavity-generated optical lattice potential is nearly perfect: the transverse profile is well characterized by the cavity modes, and in the z-axis the lattice-induced 

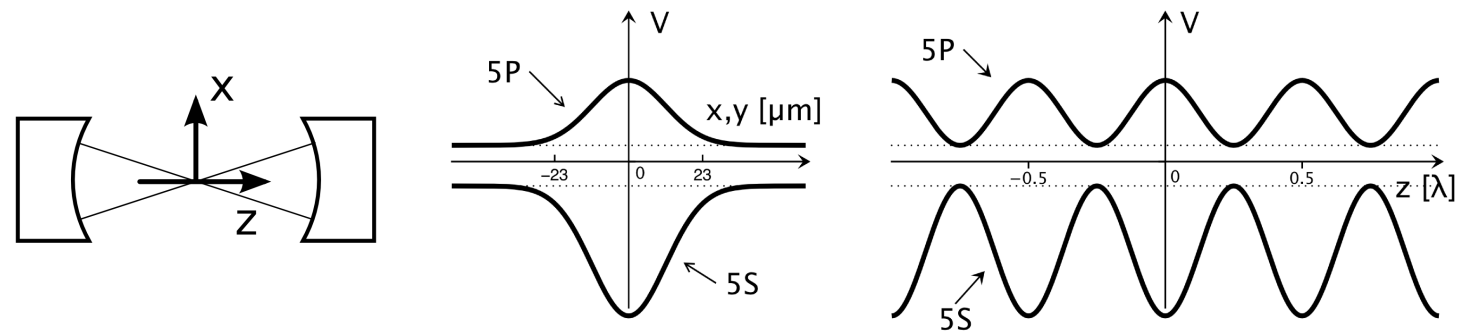

Figure 4.1:

Cavity-generated optical lattice potentials are nearly perfect. Dotted lines are unshifted energy levels, while the solid lines are lattice-shifted levels. The gap between $5 \mathrm{~S}$ and $5 \mathrm{P}$ is not to scale.

light shift cancels at the nodes. This is because the cavity mode, both in longitudinal and transverse direction, is beautiful even after the cavity is sealed inside our vacuum chamber - there is no distortion of the laser wavefront, no laser power loss or imbalance caused by any non-cavity-mirror optics, or imperfection caused by non-ideal overlap of lattice beams.

Before we move on to the discussion of experimental results, it may be worth to clarify the terms I often use in the experiment to prevent confusion.

- Lattice power: The laser power that is transmitted through the cavity mirror is always proportional to the circulating power inside. The transmitted power times $\frac{\text { finesse }}{\pi} \approx 200$ gives a good estimate of the circulating power. There is not a direct method that we can use to measure the circulating power inside the cavity. The laser intensity $I_{0}$ is calculated as,

$-I_{0}=\frac{2 P_{0}}{\pi w_{0}^{2}}$, running wave

- $I_{\text {max }}=4 \times \frac{2 P_{0}}{\pi w_{0}^{2}}$, optical lattices made of two counter propagating beams. $P_{0}$ is the single-beam power. The factor of four is because of the standing wave patterns of the lattice.

- $I_{\text {max }}=\frac{\text { finesse }}{\pi} \times 4 \times \frac{2 P_{\text {trans }}}{\pi w_{0}^{2}}$, cavity-generated. $P_{\text {trans }}$ is the transmitted lattice power. $w_{0}$ is the beam waist at the cavity center, and it is calculated from 
the beam waist of the cavity transmission, using Eq. 2.18.

The light shift experienced by the atoms at the trapping site is proportional to the lattice intensity. A better calibration of the light shift per unit of transmitted lattice laser power at the atom-trapping site can be done with two-photon excitation spectroscopic methods, which is discussed the Appendix A. For all of the experimental data in this thesis, I record the transmitted lattice power, which is simply referred to as "lattice power".

- Side camera and top cameras: The experiment has two cold-atom cloud monitoring cameras. The line of sight of one of the camera is along the x-axis, and it sees the trap center on the yz-plane, i.e. the object plane is parallel to the lattice axis. I usually call this camera the "side camera". The line of sight of the other camera is along the z-axis. This camera looks through the cavity mirror from above, and it sees the trap center on the xy-plane, i.e., the object plane is transverse to the lattice axis. I usually call this camera the "top camera".

\subsection{Fluorescence imaging}

In my experiment, I use both fluorescence imaging and absorption imaging to probe the optical lattice trap. Absorption imaging is discussed in the following section.

The top camera provides a very convenient alignment and real-time monitoring tool. Fig. 4.2 are screenshots from the top camera when the cavity is locked to $\mathrm{HG}_{00}$, $\mathrm{HG}_{10}, \mathrm{HG}_{20}$, and $\mathrm{HG}_{30}$ modes. The bright lobes inside the thin cloud of the MOT overlap the position of the cavity modes; they are the fluorescence coming from the lattice trap. In a real-time monitor window, these bright lobes are strikingly stable compared to the MOT cloud.

The light transmitted through the top cavity mirror is composed of $780 \mathrm{~nm}$ fluores- 

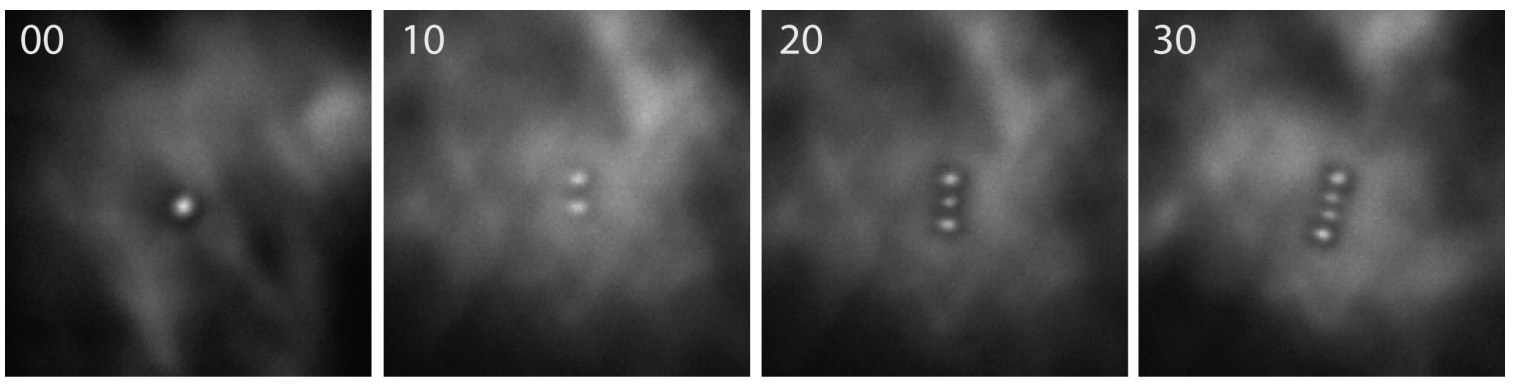

Figure 4.2:

Top camera screen shots of the bright optical lattice traps inside the thin cloud of a MOT. The cavity is locked to $\mathrm{HG}_{00}, \mathrm{HG}_{10}, \mathrm{HG}_{20}, \mathrm{HG}_{30}$ modes.

cence and $1064 \mathrm{~nm}$ lattice laser light. The coating of the cavity mirror has reflectivity at $1064 \mathrm{~nm}$, but it is approximately transparent to $780 \mathrm{~nm}$ with transmission $\sim 80 \%$. The two different wavelengths are separated by a long-pass dichroic mirror. The reflected $780 \mathrm{~nm}$ part is filtered by another low-pass filter to remove residual $1064 \mathrm{~nm}$ light before it images on the top camera. Also, the top camera looks at an angle offset from all of the MOT beams, so we expect no trace of $780 \mathrm{~nm}$ MOT light on the camera. No extra $780 \mathrm{~nm}$ probe laser exists, either. Hence, all the images in Fig. 4.2 present only fluorescence at $780 \mathrm{~nm}$. The bright lobes at the trap center might seen surprising at first, because at the trap center there is a latticed-induced light shift which should have reduced the $780 \mathrm{~nm}$ MOT fluorescence from the atoms. The bright trap center suggests that there is radiation guiding [35, 36] - the relatively far-off-resonant scattering of MOT light is guided along the mode axis (or axes) by the atom cloud, and then is captured by the top camera which is looking into the cavity mirror.

Fig. 4.3 shows fluorescence images by the top camera of the $\mathrm{HG}_{00}$ cavity mode at different lattice powers when it overlaps with the MOT. In all cases, there is a dark rim around the trap center, which we interpret as proof of the existence of a cylindrical capture volume of the lattice. MOT atoms entering the capture volume are quickly laser-cooled to the trap center, leaving a cylindrical shell which is depleted of atoms around the mode axis (or axes for higher order modes). The dark rim thus 

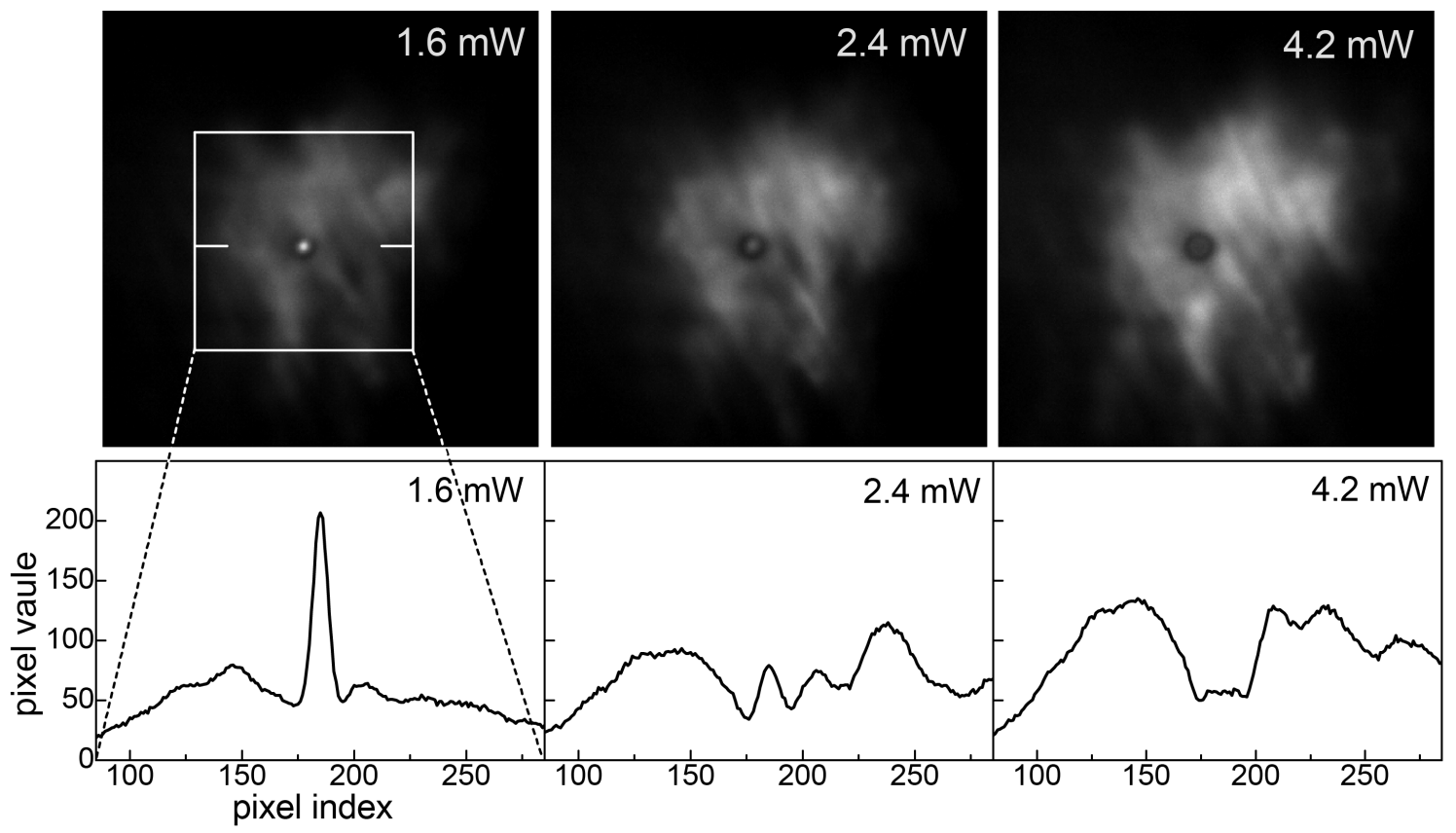

Figure 4.3:

Top: $780 \mathrm{~nm}$ fluorescence of the MOT overlapped with $\mathrm{HG}_{00}$ modes with the indicated transmitted laser powers. The exposure time of each image is $50 \mathrm{~ms}$. Bottom: Horizontal cuts of the images averaged over 3 to 5 rows of pixels. The box on the first image shows the spatial range of the cuts.

marks the boundary of the capture range of the lattice.

The bright spot observed at the center of the cavity mode disappears as the lattice power increases. This trend is observed best in horizontal cuts of the fluorescence images. At $1.6 \mathrm{~mW}$ of lattice power, the fluorescence peak, which appears Gaussian in shape, is approximately 4 times as bright as the surrounding MOT fluorescence. At $2.4 \mathrm{~mW}$, the peak is about as bright as the MOT. At $4.2 \mathrm{~mW}$, the Gaussian peak is entirely erased, leaving a flat segment at the same location.

The cavity-generated optical potential determines the atomic density distribution, which in turn determines the refractive-index profile underlying the light guiding. The atom density profile has a maximum at the mode center and rapidly decreases as a function of distance from the mode axis. Between mode axis and the surrounding MOT, there is a density gradient due to the atom-depleted region discussed above. 
This density profile results in a refractive index profile which mimics that of an optical fiber - the elongated cold-atom cloud acts as the core of the mini-fiber, with the region void of atoms acting as the cladding. An estimate of the acceptance angle of this cold-atom fiber for $780 \mathrm{~nm}$ MOT light is on the order of $1^{\circ}$, with our experimental parameters of $1.6 \mathrm{~mW}$ transmitted laser power, central volume density $\approx 2 \times 10^{11}$ per $\mathrm{cm}^{3}$, and ground-state light shift $\approx 30 \mathrm{MHz}$ [37]. The MOT light scattered by atoms near the mode axis, if emitted within the acceptance angle, could possibly be enhanced by cooperative scattering along the strongly elongated atomic cloud [38, 39]. The radiation is then guided through the elongated atomic cloud, emitted from its end, and eventually detected by the camera. The lack of radiation coming out from the trap center in the case of $4.2 \mathrm{~mW}$ may due to the lattice laser cooling limit, which is discussed in Sec. 4.4. The lattice with lattice power at $4.2 \mathrm{~mW}$ traps MOT atoms less efficiently than the lattices at lower power, and the latticeinduced light shift is larger such that the photon-scattering rate of the atoms inside the lattice is lower.

\subsection{Shadow imaging}

The lattice trap is easily observed by the fluorescence imaging due to the radiation guiding. The geometry of the trap can also be probed by absorption imaging, which also gives an estimate of atom density from the intensity absorption ratio. Details of the atom density calculation is provided in Sec. 5.2 .

Due to the lattice light shift, the transition energy of the cycling transition $5 \mathrm{~S}_{1 / 2} \rightarrow 5 \mathrm{P}_{3 / 2}$ is shifted up in frequency. To probe the atoms inside the trap with this transition, a blue-detuned, collimated, $50 \mu$ s probe is sent to the atom sample. The atoms inside the lattice see on-resonant photons and thus scatter the probe light, leaving a dark region when the probe is imaged on the CCD camera. There are both advantages and disadvantages of this blue-detuned probe scheme. The probe is only 


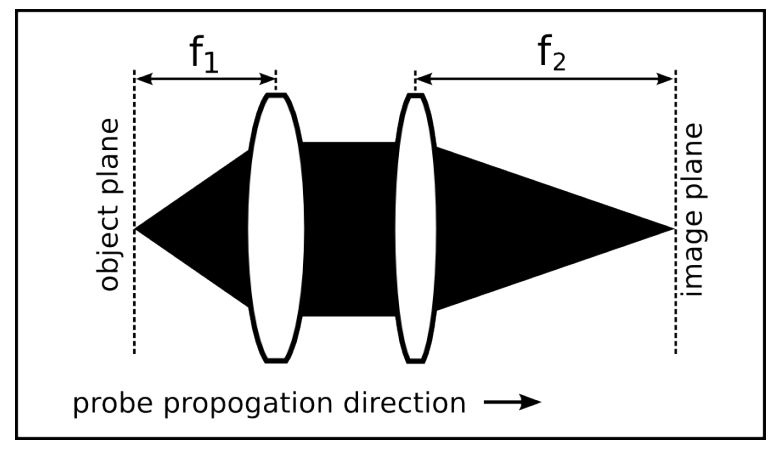

Figure 4.4: 2 f imaging: because the photon-scattering of probe light by the atom cloud is radially outward in all directions, the shadow of atom cloud images sharply on the image plane, while the probe light does not. $\mathrm{f}_{1}=15 \mathrm{~cm}, \mathrm{f}_{2}=30 \mathrm{~cm}$, the distance between the lenses is irrelevant. This lens configuration gives magnification factor of 2 .

scattered by the atoms inside the lattice where the lattice-induced lightshift matches the probe detuning, and the atoms outside the lattice are invisible in the resulting image. However, the probe only sees a portion of the lattice atoms instead of the entire population inside the lattice. With the deep lattice trap and much higher atom number density, in Chapter $\mathrm{V}$, we switch the lattice off before we send a short pulse of on-resonance probe, so the image is caused by the scattering of all the atoms, both inside and outside the lattice.

The shadow imaging in the yz-plane is done by sending a collimated probe beam with a waist much larger than the size of the MOT. The shadow of atoms images on the side camera with "2f imaging" scheme (Fig.4.4). In the xy-plan, the shadow image is obtained by co-propagating a probe beam with the lattice laser. The probe beam is divergent before entering the vacuum camber, so it is approximately collimated at the trap center. The transmission of the $780 \mathrm{~nm}$ through the cavity mirror is about $80 \%$, and the probe is separated from the $1064 \mathrm{~nm}$ laser as described in Sec. 4.2. The atom cloud shadow images onto the top comera with a 1:1 telescope and a $138 \mathrm{~mm}$ camera lens outside the chamber.

The scaling factors are: for the side camera $2.88 \mu \mathrm{m}$ in the object plane per pixel 


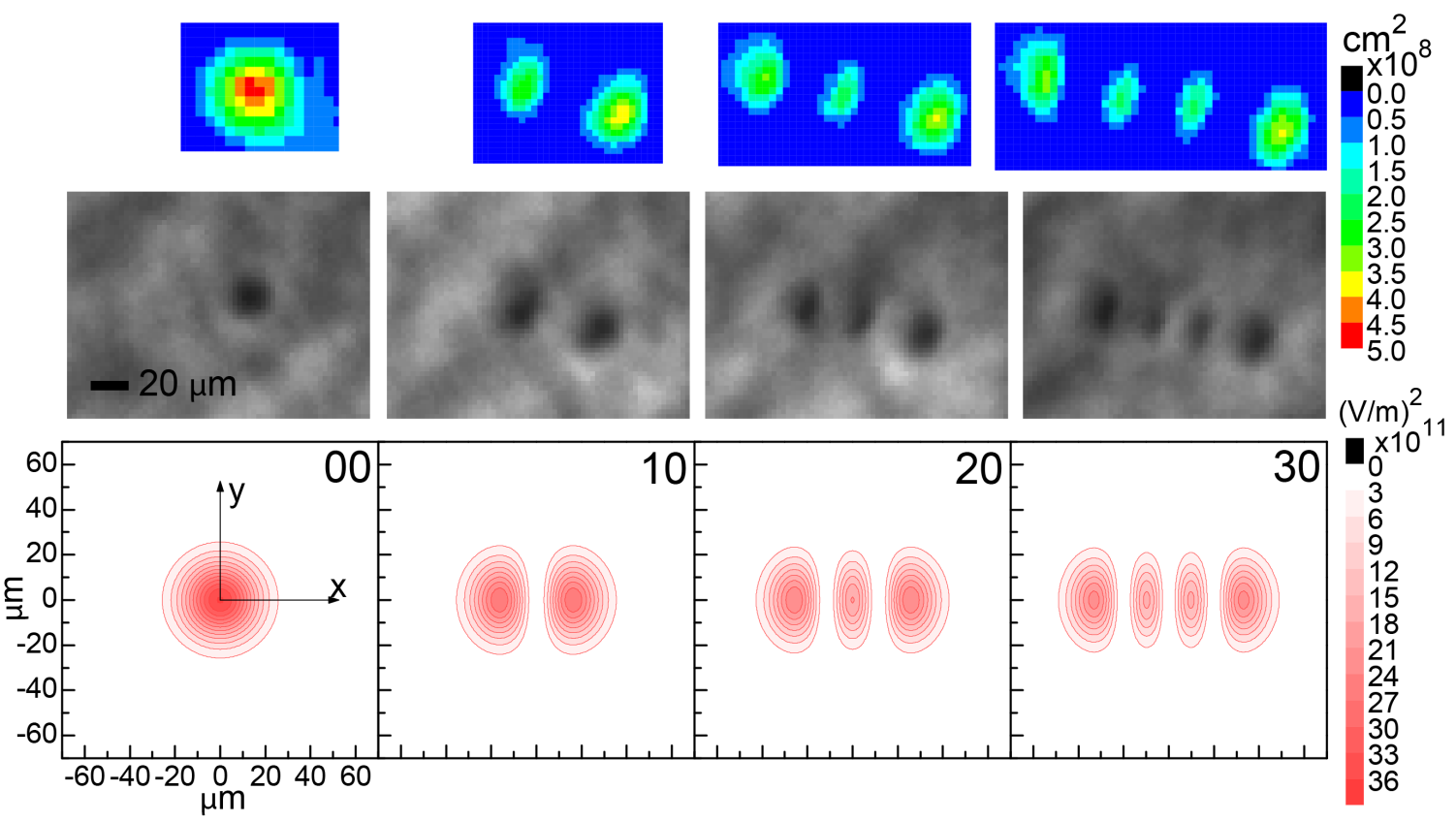

Figure 4.5:

Middle: absorption images of atoms inside the lattice fields, in the plane transverse to the lattice axis. Transmitted lattice laser powers, from left to right, are 1, 1.6, 1.9 and $1.6 \mathrm{~mW}$. The probe detuning is $27 \mathrm{MHz}$ for modes $\mathrm{HG}_{00}, \mathrm{HG}_{10}$, and $\mathrm{HG}_{20}$, and $25 \mathrm{MHz}$ for mode $\mathrm{HG}_{30}$. Top: corresponding calculated area densities of on-resonant atoms. Bottom: calculated electric-field squares of $\mathrm{HG}_{00}, \mathrm{HG}_{10}$, and $\mathrm{HG}_{20}$ and $\mathrm{HG}_{30}$, for a circulating laser power of $1 \mathrm{~W}$ and $w_{0}$ of $23 \mu \mathrm{m}$.

(Fig. 4.6, calibrated with a test object) and for the top camera $2.45 \mu \mathrm{m}$ per pixel (Fig. 4.5). The scaling factor of the top camera is obtained by comparing images of the same traps from both cameras. The alignment uncertainty of the line of sight of the side camera in Fig. 4.6 relative to the $y$-axis is $\lesssim 20^{\circ}$, as a result, the true spacings between the cavity lobes could be up to $6 \%$ more than shown by the length markers in the images. The separations between mode lobes obtained from the absorption images are in good agreement with the distances shown in the intensity profiles calculated for the Hermite-Gaussian modes, with $w_{0}=23 \mu \mathrm{m}$ derived from the cavity transmission spectrum.

The shadows of the atom clouds clearly replicate the cavity mode profile of the HG modes, which increase in overall size with mode index. For the same trap depth, 

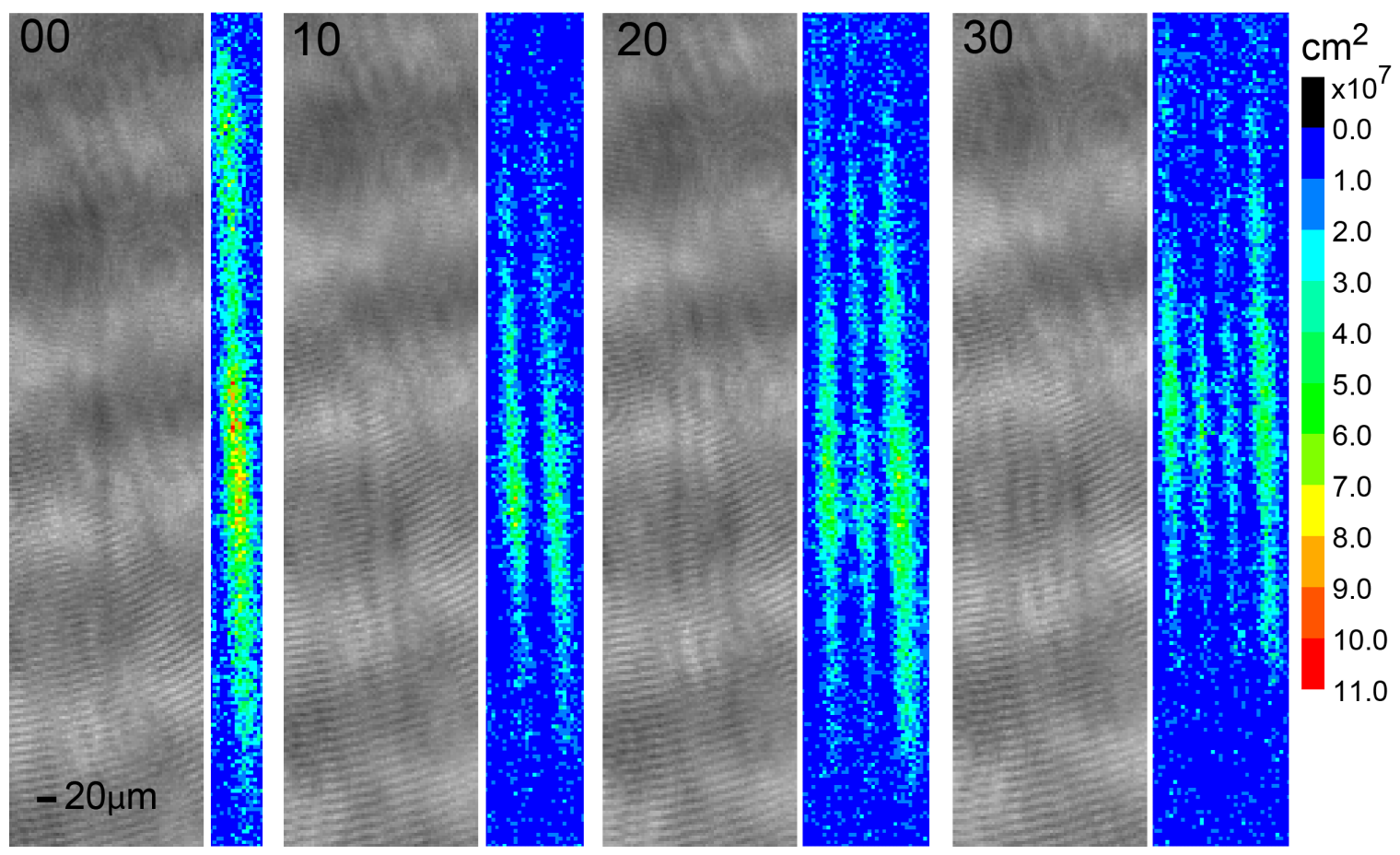

Figure 4.6:

Absorption images of atoms inside the HG lattices and corresponding area density calculation in the $x z$-plane. The aspect ratio of each cold-atom cloud is about 10 to 15 . The transmitted lattice laser powers and probe detunings are the same as in Fig. 4.5. Atom numbers computed using the on-resonant scattering cross section are 5400, 4200, 7500 and 6000, respectively; the numbers are of on-resonant atoms.

higher-order modes require higher powers, because of the overall larger trap volume (see Fig. 4.5). Both Fig. 4.5 and 4.6 (top view and side view) show that the lobe spacing decreases with mode number. Also, the outermost lobes form deeper traps are darker than the inner ones.

The periodicity of the lattice in $z$ direction is $532 \mathrm{~nm}$, which cannot be resolved by the side camera. As a result, the atom density calculation in the z-axis is an average over this axis. The atoms density inside a single lattice potential well is twice or higher than the average.

The absorption images show that we can realize clean, stable $1 \mathrm{D}, 2 \mathrm{D}$ and $3 \mathrm{D}$ trapping geometries for ultra-cold atoms. The cavity functions as a mode filter to implement a variety of higher-order modes with well-defined, precisely known, regular 
patterns and lobe spacings.

Absence of cavity mode-mixing is ensured by a sufficient degree of non-degeneracy in the cavity transmission spectrum (see Fig. 2.3). The input laser is directed into the cavity in a manner such that the first few Hermite-Gaussian cavity modes form clean spectral peaks on the transmission spectrum, and thus the electronic feedback circuit selectively stabilizes the cavity to only one of the cavity mode.

\subsection{Spectroscopy and lattice laser cooling limit}

The lattice cooling limit is determined by two-photon spectroscopy of Rydberg 60D levels. Details of the signal acquisition with a microchannel plate detector, and the stray field compensation with Stark spectroscopy are described in Chapter III. The spectroscopy here is done by locking the cavity to the $\mathrm{HG}_{00}$ mode, frequency-stabilizing the lower transition laser to the cycling transition $5 \mathrm{~S}_{1 / 2} \rightarrow 5 \mathrm{P}_{3 / 2}$, blue-shifting the lower transition beam with an AOM, and then scanning the upper transition laser. The experiment runs at $100 \mathrm{~Hz}$, the lattice laser is always on, the excitation pulse is on for $10 \mu \mathrm{s}$, and the Rydberg atoms are field-ionized at the end of each experimental cycle. The excitation lasers intersect at the trap center; the upper transition has a focus of FWHM $40 \mu \mathrm{m}$ and a power $\lesssim 0.5 \mathrm{~mW}$, and the upper transition laser is collimated to a size that is larger than the MOT with intensity $1 \mathrm{~mW} / \mathrm{cm}^{2}$, which is about $\frac{1}{4} I_{\text {sat }}$ (saturation intensity). This configuration of excitation laser parameters allows us to clearly observe both the on-resonantly excited lattice atoms and off-resonant MOT atoms on a single spectroscopic scan.

For a shallow lattice, i.e. the AC Stark shift caused by the lattice light field is weak compared to the hyperfine structure, and the atomic energy level is altered according to $-\frac{1}{4} \alpha E_{0}^{2}$, where $\alpha$ is the dynamic polarizability. In a $1064 \mathrm{~nm}$ optical lattice, $\alpha_{5 \mathrm{~S}}=4 \pi \varepsilon_{0} \times 687.3 a_{0}^{3}$ [40], $\alpha_{5 \mathrm{P}}=\alpha_{0}+\alpha_{2} \frac{3 m^{2}-F(F+1)}{F(2 F-1)}$, with $\alpha_{0} \approx-4 \pi \varepsilon_{0} \times 1120 a_{0}^{3}$ and $\alpha_{2} \approx 4 \pi \varepsilon_{0} \times 520 a_{0}^{3}$ (see Chapter VII for the details of $\alpha_{0}$ and $\alpha_{2}$ ). For Rydberg 
(a)

transmitted laser power: $1.2 \mathrm{~mW}$

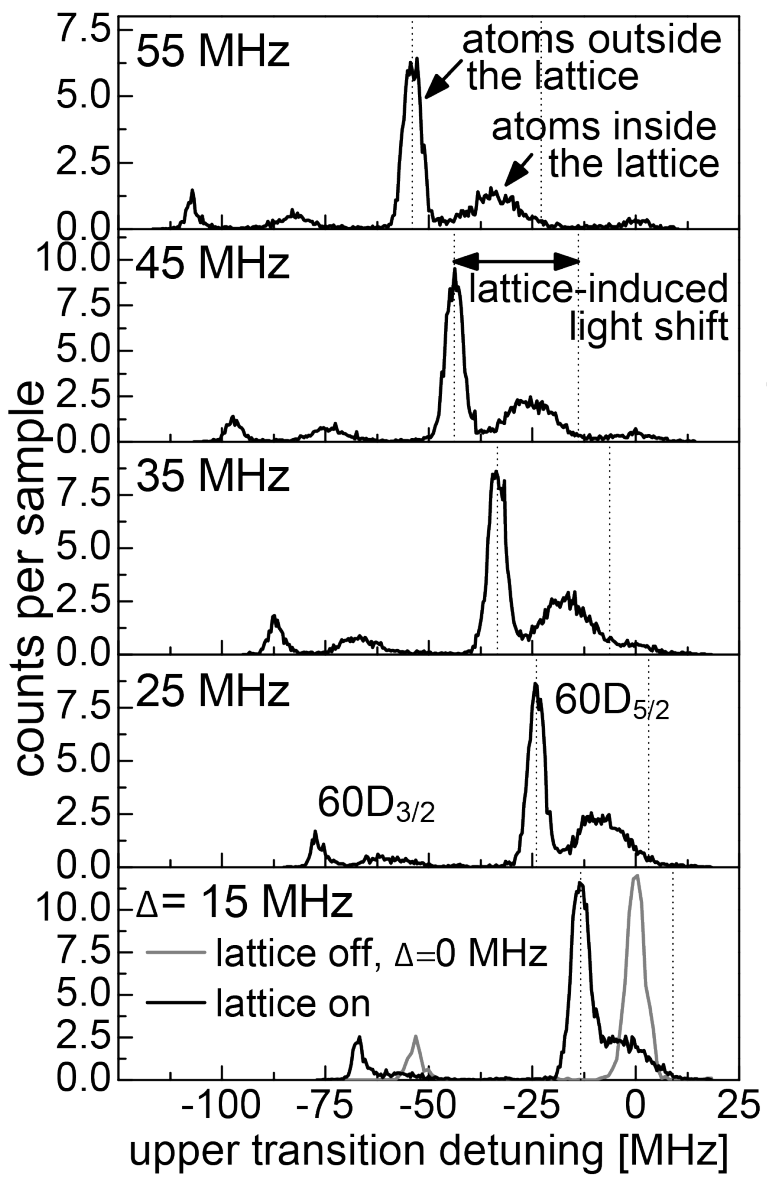

(b)

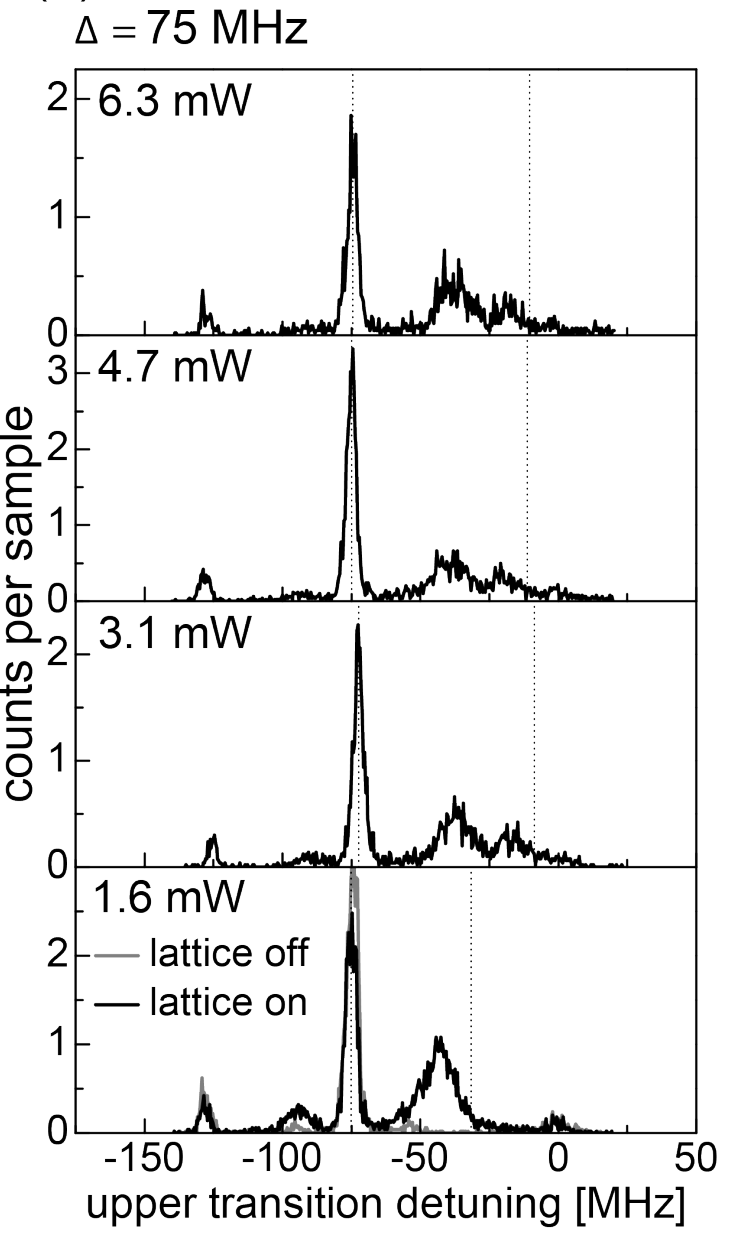

Figure 4.7:

Two-photon excitation spectra of $\mathrm{Rb} 60 \mathrm{D}$ levels. Spectral resolution is $\approx 5 \mathrm{MHz}$. (a) Spectra with transmitted lattice laser power $1.2 \mathrm{~mW}$, and the indicated lower-transition detunings $\Delta$. (b) Spectra for the indicated transmitted lattice laser powers and $\Delta=75 \mathrm{MHz}$. The experimental repetition rate is $100 \mathrm{~Hz}$. Each data point is an average of 50 shots. The small signal at $0 \mathrm{MHz}$ is likely due to MOT leakage light.

atoms, $\alpha_{\mathrm{R}}$ results from the free-electron ponderomotive shift $-4 \pi \varepsilon_{0} \times 545 a_{0}^{3}$ [19]. The actual value of $\alpha_{r m R}$ depend on all quantum numbers of the utilized Rydberg levels and where the atom is located relative to the lattice axis. The sign of the polarizability determines the polarity of the energy level shift in the lattice. In the $1064 \mathrm{~nm}$ lattice, i.e., 5S level has potential minima coincident with the lattice intensity minima, while the $5 \mathrm{P}$ and the $60 \mathrm{D}$ Rydberg levels have potential maxima coincident 
with the intensity maxima.

In order to near-resonantly excite the atoms inside the lattice to 60D levels, the lower transition laser is blue-detuned by $\Delta(\Delta>0)$ relative to the field-free cycling transition (of ${ }^{85} \mathrm{Rb} 5 \mathrm{~S}_{1 / 2} \mathrm{~F}=3 \rightarrow 5 \mathrm{P}_{3 / 2} \mathrm{~F}^{\prime}=4$ ); $\Delta$ is within the range of the cavitygenerated light shifts of that transition. The corresponding upper-transition detuning becomes $-\Delta$ plus the lattice-induced light shift of the two-photon $5 \mathrm{~S}_{1 / 2} \mathrm{~F}=3 \rightarrow 60 \mathrm{D}$ transition. The narrow peaks in Fig. 4.7, located at upper-transition detuning $-\Delta$, result from off-resonant excitation of atoms outside the lattice, whereas the broadened blue-shifted features result from the atoms inside the lattice. Therefore, the frequency distances between the narrow lines and the blue-shifted features reveal the lattice-induced light shifts [22]. The maximal shift is estimated by the distance from the narrow $60 \mathrm{D}_{5 / 2}$ line to the high-frequency edge of its blue-shifted feature, represented by the dashed lines in Fig. 4.7. This light shift is a combination of the shifts of $5 \mathrm{~S}_{1 / 2}$ and of $60 \mathrm{D}_{5 / 2}$, which can be calculated using known dynamic polarizabilities. Roughly, $5 \mathrm{~S}_{1 / 2}$ accounts for $2 / 3$ of the total shift, and $60 \mathrm{D}_{5 / 2}$ for $1 / 3$. For example, in the top panel of Fig. 4.7(a), the maximal lattice-induced light shift is about $30 \mathrm{MHz}$, corresponding to the asymptotic value in Fig. 4.8(a). Therefore, for a transmitted lattice power of $1.2 \mathrm{~mW}$ the lattice potential depth for ground-state atoms is $\approx 20 \mathrm{MHz}$.

Fig. 4.7(b) shows the spectra for higher transmitted lattice laser powers. We set $\Delta$ to $75 \mathrm{MHz}$ to be able to probe deeper optical lattices. Generally, we find for transmitted powers below approximately $2 \mathrm{~mW}$ that the maximal induced light shift scales linearly with the transmitted power. We conclude that in this power range the atoms are laser-cooled to the bottom of the optical lattice, and the calibration factor relating the $5 \mathrm{~S}_{1 / 2}$ lattice depth and the transmitted lattice laser power is $\approx$ $18 \mathrm{MHz}$ per $\mathrm{mW}$ for the fundamental Hermite-Gaussian mode, $\mathrm{HG}_{00}$. However, for transmitted powers larger than $2 \mathrm{~mW}$, the measured light shift reaches a maximum 

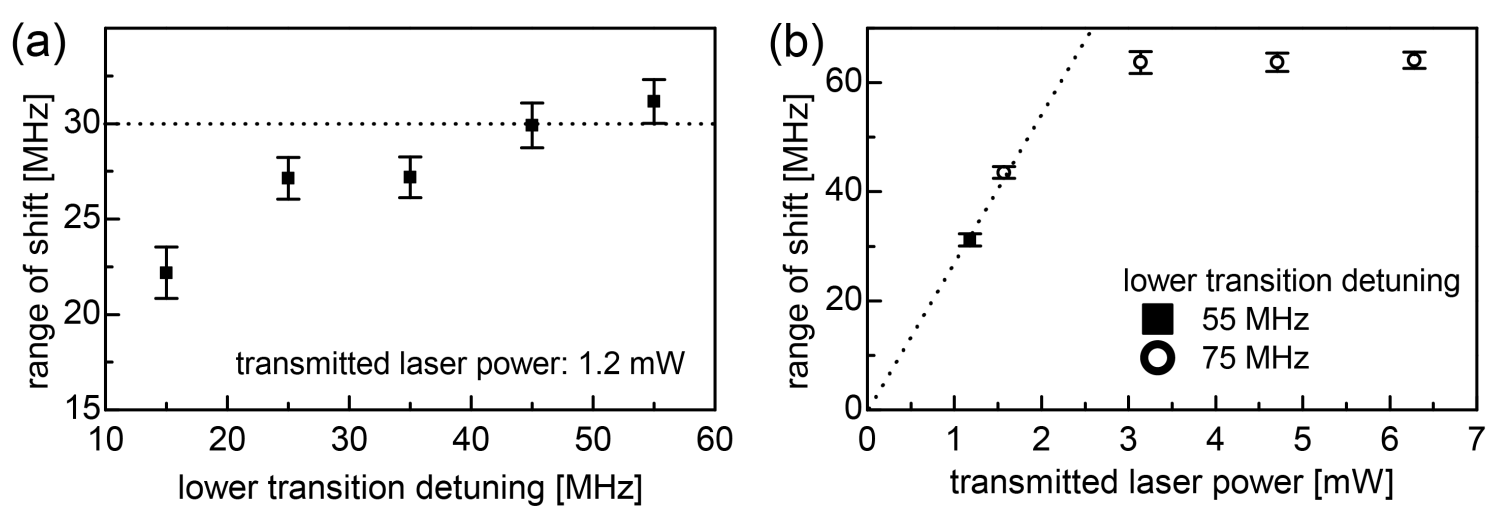

Figure 4.8:

Maximal upper-transition shifts of the $60 \mathrm{D}_{5 / 2}$ level from Fig. 4.7 (a) vs lower-transition detuning $\Delta$ and (b) vs transmitted laser power. The amount of shift is estimated by the frequency distance between the central position of the narrow line to the broadened blue-shifted feature, plus half of the $1 / e^{2}$ full width of Gaussian fits to the blue-shifted feature. In the cases that the blue-shifted feature shows multiple peaks, the one at larger detuning is used. The dotted line in (a) indicates that the lattice induced shift converges to an asymptotic value of about $30 \mathrm{MHz}$. The straight line in (b) shows that at low powers the calibration for the ground-state atom trap depth is about $18 \mathrm{MHz} / \mathrm{mW}$.

at $\approx 60 \mathrm{MHz}$ and the ground-state trap depth is about $40 \mathrm{MHz}$ (see Fig. 4.8(b)), showing that the atoms are not cooled to the bottom of the optical lattice in this power regime. To overcome the lattice cooling limit, we have the lattice adiabatic compress the atom sample. The experimental details are discussed in Chapter V. and the calibration factor relating the lattice-induced light shift in a deep lattice is provided in Appendix A.

The substructures seen in some of the higher-power cases are due to the $m$ dependence of the $5 \mathrm{P}_{3 / 2}$ light shifts, which lead to resonant enhancement of the signal at several upper-transition detunings. In Chapter VII I present the measurement of polarizability in the strong lattice laser field of the deep optical lattice, where the substructures are resolved and employed to measure polarizabilities.

Spectra for higher-order HG modes are qualitatively similar to these shown in Fig. 4.7. For higher-order modes the blue-shifted signal which arises from the lattice 
trap is stronger because of the larger trap volume, and the calibration factors for the trap depths per transmitted power are lower.

\subsection{Ionization of ground-state rubidium atoms}

We use a MCP detector to detect the field-ionized Rydberg atoms in most cases. However, in the following discussion, I focus on an ion source that does not originate from optical Rydberg excitation but from a rather different mechanism.

We consistently observe blips of background ions on the phosphor screen of the MCP detector, as long as the $1064 \mathrm{~nm}$ lattice trap and the weak extraction electric field are both present. These background ions are independent of the $480 \mathrm{~nm}$ laser; no direct optical production of Rydberg atoms is needed. In this case, the ions detected by the MCP are unlikely to be induced by field-ionization, since the weak extraction field is far below the strength required to ionize the ground-state Rb atoms. The count rate of detected ions is negligible compared to the strength of Rydberg spectral lines, but the blips of the ions are very well localized on the MCP phosphor screen, such that we suspect these ions could have resulted from a combination of collision and photoionization processes involving both the $780 \mathrm{~nm}$ and the $1064 \mathrm{~nm}$ laser light.

To characterize the spatial distribution of these ions, the videos of the phosphor screen of the MCP detector are averaged over many shots. Fig. 4.9 shows averaged video frames of the phosphor screen when the cavity is locked to $\mathrm{HG}_{00}$ and $\mathrm{HG}_{10}$ modes, with lattice power at about $1.5 \mathrm{~mW}$. In order to launch the ions to the MCP, the electric field is continuously on with a strength of $56 \mathrm{~V} / \mathrm{cm}$ at the cavity center. The blips on the phosphor screen reflect the spatial distribution of the ions. The dark counts of the MCP are randomly distributed on the phosphor screen, so they are almost completely eliminated after averaging over many frames. The blips due to ions are very localized on the phosphor screen, so the signal peaks up after averaging. For the case of the $\mathrm{HG}_{00}$ cavity mode, the ions come from a single source. For the 


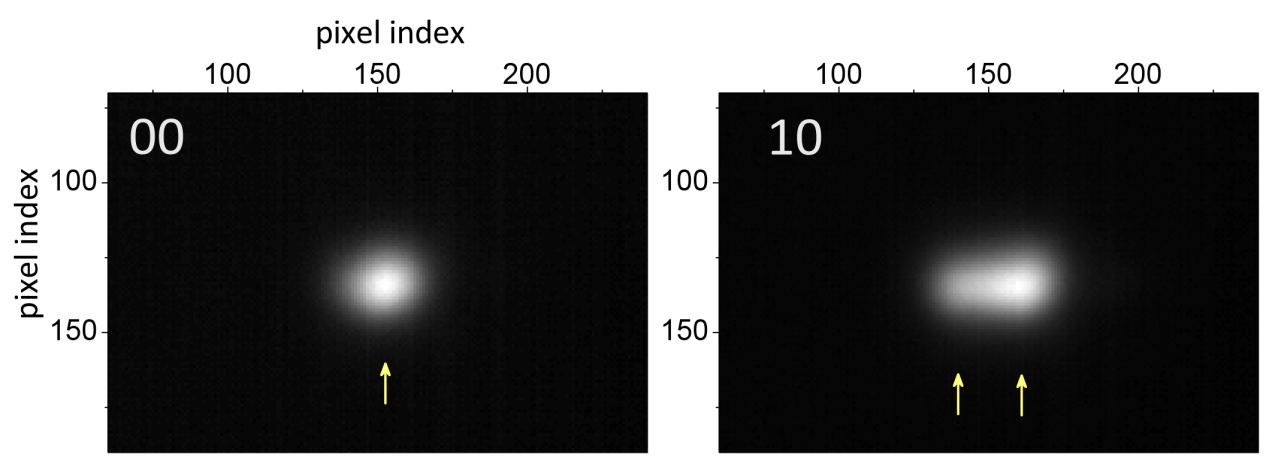

Figure 4.9:

Average of the blips on MCP phosphor screen when cavity is locked to $\mathrm{HG}_{00}$ mode and $\mathrm{HG}_{10}$ modes. The blips reflect the spatial distribution of the cavity modes. $\mathrm{HG}_{00}$ : average of 800 shots, $\mathrm{HG}_{10}$ : average of 1000 shots. The image of the $\mathrm{HG}_{10}$ mode shows the ions originate from two sources whose spacing is about or less than $20 \mu \mathrm{m}$ (i.e. the lobe spacing of the $\mathrm{HG}_{10}$ cavity mode, see Figure Fig. 4.5.

case of the $\mathrm{HG}_{10}$ cavity mode, the averaged image shows two maxima. The cavity mode $\mathrm{HG}_{10}$ is oriented such that the two elongated cold-atom clouds both face the MCP, and the ions emerging from the two sources are resolved on the MCP phosphor screen 1 . This spatial structure provides evidence that the ionization occurs inside the $1064 \mathrm{~nm}$ lattice trap.

To determine the laser that may be responsible for these observations, the suspected lasers are programmed to pulse on an off at different times, and the detected ions are counted as a function of time in an experimental cycle. In each single cycle of an experiment, a device called multichannel scaler (Stanford Research Systems SR 430) partitions the detected ion counts into sequential bins depending on when the counts arrive. The multichannel scaler also allows repeating the experiment for several thousand times and summing over the results; the accumulation of scans increases the signal to noise ratio of a real event, and reduces the effect of laser or MOT density drifts.

Fig. 4.10(a) shows that the count rate immediately drops to the background level

\footnotetext{
${ }^{1}$ In the MCP phosphor screen shots, the two sources are resolved in the horizontal direction, because there is magnification in this direction due to the geometry of the electrodes surrounding the cavity. There is no magnification in the vertical direction
} 
when either the MOT laser or lattice laser is pulsed off. In Fig. 4.10(b), the MOT light, including the cycling transition laser and repumper laser, is pulsed off for $1 \mathrm{~ms}$, and a short pulse of probe tuned to the cycling transition occurs at a different time in each of the scans. The count rate is immediately high again when the the probe is on. These two sets of scans conclude that the ionization requires both the cycling transition laser at $780 \mathrm{~nm}$ and the $1064 \mathrm{~nm}$ lattice laser.

The photoionization of vaporous $\mathrm{Rb} 5 \mathrm{~S}_{1 / 2}$ ground-state atoms can be off-resonantly induced by two photons with wavelengths in range $540 \mathrm{~nm}$ to $590 \mathrm{~nm}$ [41], when the photoionization laser intensity is strong. In my experiment, the laser with the strongest intensity is the far-off-resonant $1064 \mathrm{~nm}$ lattice laser, whose strength is on the order of $10^{11} \mathrm{~W} / \mathrm{m}^{2}$ and is likely not strong enough to drive an off-resonant photonization on its own. There are two possible mechanisms that may cause the ionization of ground-state rubidium atoms, both of which are based on Penning ionization,

1. The $1064 \mathrm{~nm}$ laser is directly involved in the ionization. The $780 \mathrm{~nm}$ cycling
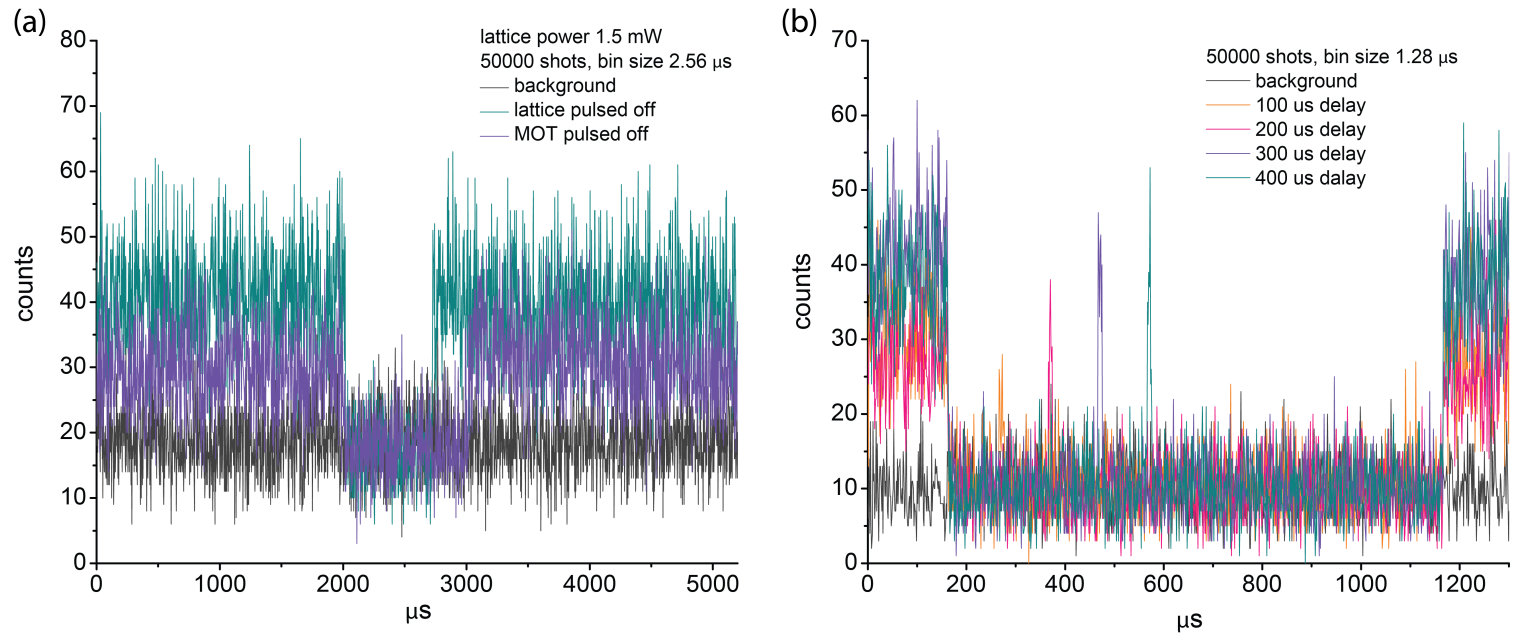

Figure 4.10:

Photoionization multichannel scaler scans. (a) Photo-ionization count rate drops when either MOT light (including cycling and repumping transitions) or lattice laser is turned off. (b) Count rate returns to high level when the $10 \mu$ s probe (cycling transition $5 \mathrm{~S}_{1 / 2} \rightarrow 5 \mathrm{P}_{3 / 2}$ ) pulses on. 
transition laser excites the ground-state $\mathrm{Rb}$ atoms from $5 \mathrm{~S}_{1 / 2}$ to $5 \mathrm{P}_{3 / 2}$, and then two excited-state $5 \mathrm{P}_{3 / 2}$ atoms collide in the presence of a $1064 \mathrm{~nm}$ photon, which collectively exceeds the energy threshold required to achieve the ionization. In brief,

$5 \mathrm{P}_{3 / 2}+5 \mathrm{P}_{3 / 2}+1064 \mathrm{~nm} \gamma \rightarrow \mathrm{Rb}^{+}+5 \mathrm{~S}_{1 / 2}+\mathrm{e}^{-}+$kinetic energy.

2. The $1064 \mathrm{~nm}$ laser is not directly involved in the ionization, but it provides a high enough density of trapped atoms such that the $780 \mathrm{~nm}$ cycling transition off-resonantly excites a significant amount of 5D atoms. The ions may then result from Penning ionization involving the collision of atoms at $5 \mathrm{P}$ and $5 \mathrm{D}$ excited states.

$5 \mathrm{P}+5 \mathrm{D} \rightarrow \mathrm{Rb}^{+}+\mathrm{Rb}+\mathrm{e}^{-}+$kinetic energy

Further multichannel scaler scans would be required to determine which mechanism causes the ionization process observed.

Regardless of the ion-production mechanism, this could be used in MOT lasercooled ion sources, which are used in the fabrication and microscopy of nanoscale materials [42], and they are advantageous over other ion sources for low beam energy and sputtering. The ion source originating from atoms trapped in cavity-generated optical potentials could have further advantages - spatial control of the ion sources could be achieved by stabilizing the cavity to the cavity mode which gives the desired number and geometry of cold-atom channels. As shown in Fig. 4.9, the angular spread of the ion beam is minimal, and we can still resolve the two $\mathrm{HG}_{10}$ channels on the MCP, which is about 5 inches away from the lattice trap. 


\section{CHAPTER V}

\section{Lattice adiabatic compression}

In this chapter, I present the experimental details of lattice adiabatic compression, which is a critical technique in my experiment. The lattice adiabatic compression allows us to overcome the lattice cooling limit and create lattice light shift on the order of several GHz.

\subsection{Advanced cavity stabilization}

In my experiment, I use a cw, narrow-band $1064 \mathrm{~nm}$ fiber laser as the lattice laser (IPG photonics YLR-10-1064-LP-SF). The 1064 nm laser has a short term linewidth $<100 \mathrm{kHz}$, as specified by the manufacturer. However, the output of the $1064 \mathrm{~nm}$ fiber laser for the lattices is not perfectly noise-free for the use in my experiment. This is noticed by daily observations on the cavity transmission spectra - when the cavity length is in scan mode, the peaks on the transmission spectrum are not entirely stationary; there are long-term drift and short-term fluctuation. A frequency drift would not significantly affect the trapping of atoms because of the adiabatic following; the atoms keep trapped because the time scale of the frequency drift is relatively slow to the atomic motion in optical dipole traps or optical lattices. However, the same frequency drift poses problems for the concentric cavity experiment, because the frequency drift results in the intracavity intensity fluctuation. 


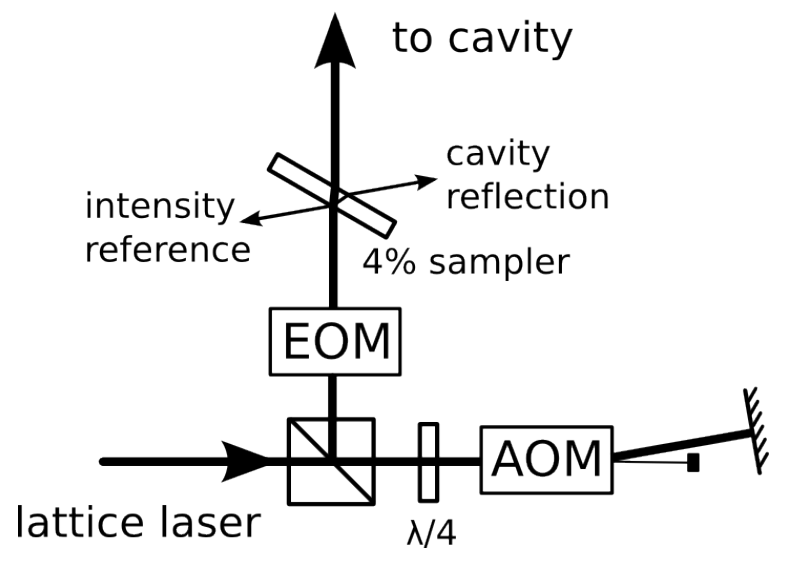

Figure 5.1: Optics layout for the Pound-Drever-Hall stabilization scheme with lattice amplitude modulation. See text for details. EOM: electro-optic modulator. AOM: acousto-optic modulator.

With the cavity locked to the laser, the slow drift changes the percentage of input laser power coupled into the cavity, which in turns affects the intracavity and transmitted lattice power on a long time scale. However, the slow drift does not significantly affect the PDH cavity stabilization scheme. The short term frequency fluctuation of the $1064 \mathrm{~nm}$ laser is the most concerning. The short-term fluctuation can also be seen when the cavity length is in scan mode. The peaks shake left-right on the transmission spectrum, indicating that the laser frequency fluctuates. When the electronic feedback circuit is in lock mode, this frequency fluctuation causes an undesirable transmitted-power fluctuation, which indicates a corresponding fluctuation of the lattice trapping potentials. This results in unwanted signal-to-noise reduction and spectral line broadening of the signals of atom inside the lattice.

To minimize the transmitted power fluctuation, I insert a double-pass AOM in the lattice laser beam path, using the AOM's RF frequency modulation to compensate the short-term frequency fluctuation. A schematic diagram is shown in Fig. 5.1. The philosophy here is similar to the frequency stabilization of an ECDL: the output wavelength is stabilized by the slow movement of the grating, but the linewidth is reduced further by the fast modulation of the laser diode current. I use the relatively 
fast frequency modulation of the AOM to compensate the $1064 \mathrm{~nm}$ laser's frequency fluctuation.

Compared to the common cavity PDH stabilization scheme, as shown in Sec. 2.3 . the main differences of this combined stabilization scheme are as follows: first, most of the $1064 \mathrm{~nm}$ input is deflected into the -1 order twice, and then passed through the EOM. All the other orders, including the zero order, are blocked. Second, the $\mathrm{PDH}$ error signal which is generated with the EOM and PDH module, is sent to both the PID feedback module (for piezo lock) and a proportional feedback circuit. The stabilization of the cavity still relies on the high-voltage PID feedback to the ring piezo (slow feedback component), but with an added laser AOM frequency modulation controlled by the proportional feedback circuit (fast feedback component). The frequency of AOM-deflected laser beam is modulated by means of modulating the RF frequency applied to the crystal inside the AOM. The proportional feedback circuit controls the RF frequency modulation in a manner such that the frequency-shift of the deflected beam compensates the frequency fluctuation of the fiber laser. Third, in addition to the frequency modulation, the amount of input laser sent to the cavity is also modulated by modulating the RF power that drives the crystal inside the AOM. The amplitude of the lattice power sent to the cavity scales with the RF power output of the AOM driver, which in turns is voltage-controlled by an envelope generated by an arbitrary function generator. In later experiments with the deep lattice trap with experimental repetition rate around $100 \mathrm{~Hz}$, we can program the arbitrary function generator to produce an envelope such that the lattice laser power is kept constantly low most of the time, and then increased for a short period in each experimental cycle.

Fig. 5.2 shows a scope screenshot of the cavity transmitted power (yellow line), which is proportional to the lattice laser intensity at the cavity center. The lattice intensity is increased by a factor of 20 within a ramp time of $30 \mu \mathrm{s}$, then held high 


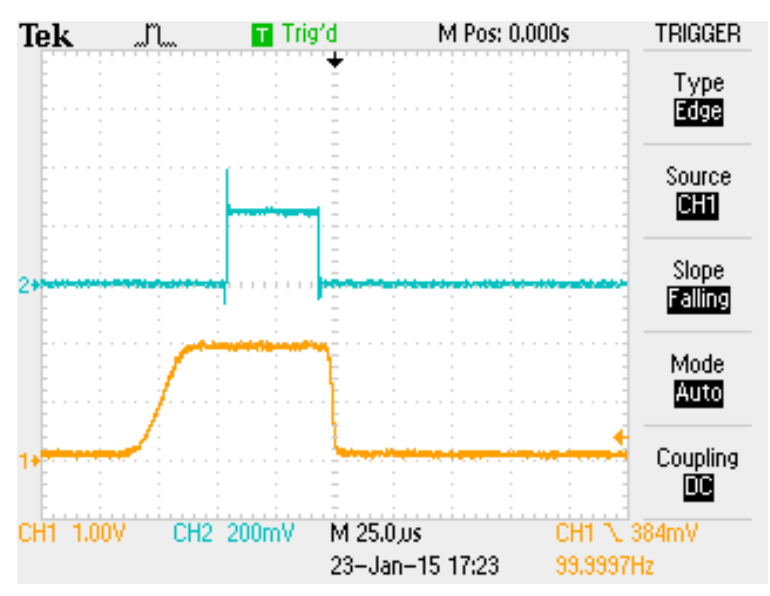

Figure 5.2:

A screenshot of cavity transmitted power (yellow) monitor by a photodiode. Blue curve is a multiplexer pulse.

for another $70 \mu \mathrm{s}$, and finally returns to low power at the end of an experimental cycle. This intensity stepping reduces the chance of damaging the in-vacuum optics by excessive heating by the fiber laser. The intensity stepping also allows us to load the lattice at a shallow lattice potential, and then adiabatic compress the atom samples to overcome the lattice laser cooling limit, which is discussed in the previous Sec. 4.4 .

\subsubsection{Real-time normalization circuit}

The amplitude of the PDH error signal scales with the amplitude of cavity reflection, which itself is proportional to the input laser power. The modulation of the laser power by the AOM thus causes an inevitable modulation of the PDH error signal amplitude. Both the slow piezo-lock feedback circuit and the frequency modulation feedback circuit that take this PDH error signal have fixed gain; the gain is manually controlled by analog trimpots. The change of the PDH error signal amplitude without an automatic control of the gain causes an overshooting of the feedback circuits, and the cavity transmission oscillates madly whenever the feedback circuits overshoot. To prevent the feedback circuits from seeing a varying amplitude of error signal, I add a 
PDH error signal real-time normalization circuit, as shown in Fig. 5.4.

Common PID feedback circuits are realized by operational amplifiers (op amp): with a combination of resistors and capacitors, one can make the output of the op amp proportional to the input, or to be an integration or a differentiation of the input signal. With the use of diodes, as shown in Fig. 5.3, the op amp can also perform logarithmic output or exponential output. For the logarithmic output, $V_{\text {out }}=$ $-V_{T} \ln \left(\frac{V_{i n}}{I_{S} R}\right)$, and for the exponential output, $V_{\text {out }}=-R I_{S} \exp \left(\frac{V_{i n}}{V_{T}}\right) . V_{T}$ is the thermal voltage, and $I_{S}$ is the saturation current of the diode.

The real-time PDH error signal normalization circuit is composed of log amplifiers, exponential amplifiers, and summing amplifiers. The circuit takes logarithms of the input signals, and them performs adding/subtraction operation with the summing amplifier. The output of the summing amplifier is amplified by the exponential amplifier. In this manner, the circuit output voltage is,

$$
\text { output }=\text { amplitude } \times \frac{\text { PDH error signal }}{\text { reference signal }}
$$

The reference signal is obtained by monitoring the $4 \%$ reflection from the beam sampler at the input side (see Fig. 5.1). The only non-straight-forward part is adjusting the time constant of the low-pass filter for the reference photodiode which monitors the $4 \%$ reflection. The photodiode has a relatively slow rising time for the purpose of reducing noise. The time constant of the low-pass filter needs to be adjusted to
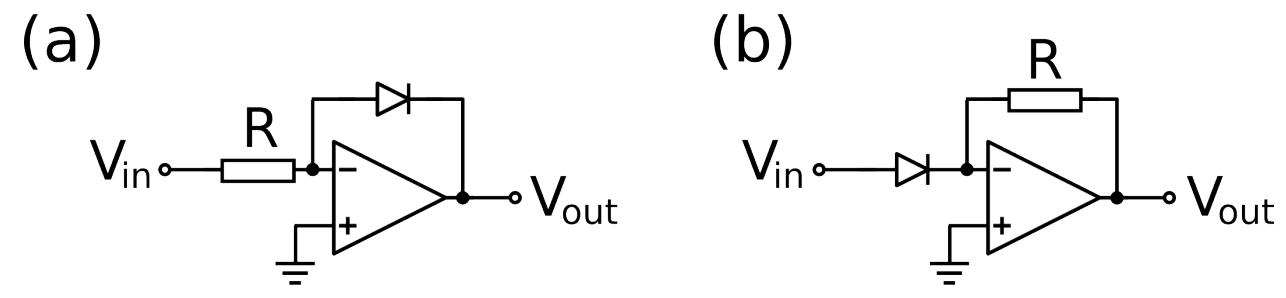

Figure 5.3: (a) logarithmic output. (b) exponential output. 


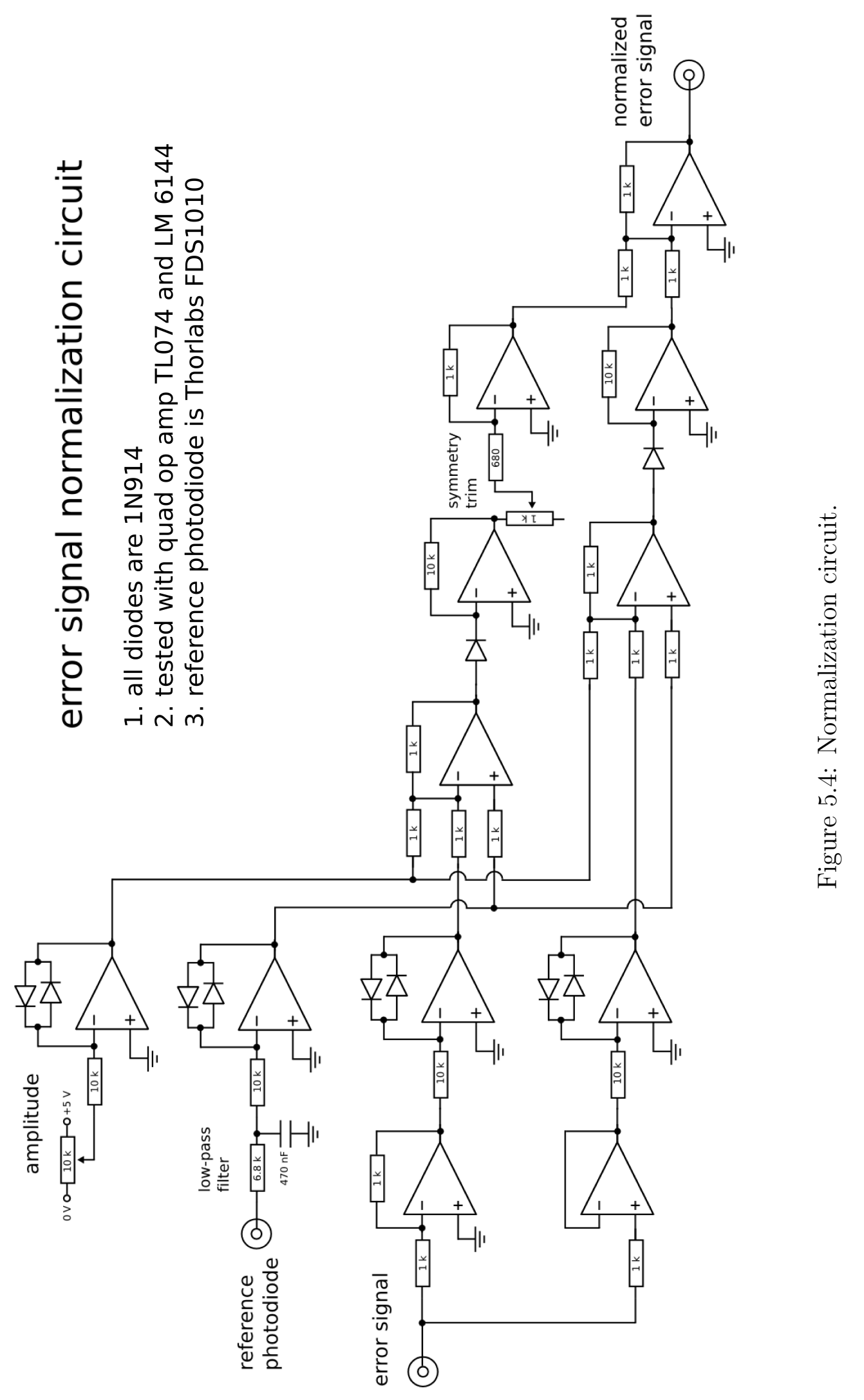


optimize the normalization performance and minimize the residual PDH error signal modulation.

\subsection{Atom density estimation}

The densities of atom samples inside the lattice traps are estimated by absorption imaging, which is mostly called "shadow imaging" in lab. The lattice axis is the zaxis in my experiment, and the imaging is on yz-plane. A probe beam, with intensity $I_{0}(y, z)$, shines through the atom sample on yz-plane right after the lattice light is turned off, and then the remaining intensity $I(y, z)$ is imaged onto a CCD camera (the "side camera") whose line of sight is along the x-axis. The area density is calculated from the change of intensity of the probe beam due to the photon scattering by atoms. When the atom sample is dense, the portion of the probe that passes through dense area is almost extinguished, leaving a "shadow" on the images of the bright probe field.

Over a length element $d x$, the change of probe intensity, $I(y, z)$, is,

$$
d I=-\gamma h \nu n_{V}(x, y, z) d x
$$

where $n_{V}$ is the volume density of the atom sample, $\gamma$ is the scattering rate, and $h \nu$ is the transition energy of the levels involved in the photon-scattering.

In the case of low probe intensity $\left(I<I_{\text {sat }}, I_{\text {sat }}\right.$ saturation intensity), and a probe

frequency on-resonance with the $5 \mathrm{~S} \rightarrow 5 \mathrm{P}$ transition, the scattering rate $\gamma$ equals $\frac{\Gamma I}{2 I_{s a t}}$, $\Gamma$ is the decay rate of the transition. Eq. 5.1 is rearranged to get,

$$
n_{V}(x, y, z) d x=-\frac{2 I_{s a t}}{\Gamma h \nu} \frac{d I}{I}
$$


Integrating both side of the equation gives the area density $n_{A}$,

$$
n_{A}(y, z)=\frac{2 I_{s a t}}{\Gamma h \nu} \ln \frac{I_{0}}{I}
$$

$I_{0}$ is the initial intensity of the probe beam, and $I$ is the intensity after the probe beam passes the atom cloud.

Experimentally, the probe beam is unpolarized, and is tuned on-resonance with the MOT cycling transition. The camera takes three images for each round of calculation: one image with the probe beam passed through the atom sample $(I)$, one image with the probe beam when there is no sample $\left(I_{0}\right)$, and a background image when both probe beam and atom sample are off $\left(I_{B}\right)$. For a Rb atom sample and probe beam tune to the MOT cycling transition, the area density of each pixel on the image is calculated by,

$$
n_{A}(y, z)=3.44 \times 10^{8} \times \ln \frac{I_{0}(y, z)-I_{B}(y, z)}{I(y, z)-I_{B}(y, z)} \mathrm{cm}^{-2}
$$

Fig. 5.5 shows a sample plot of the area density of the lattice trap obtained from the shadow image on the yz-plane. The trap looks like a needle. The observation is not surprising in light of the geometry of the cavity-generated trapping potential: the intensity profile of the cavity mode is Gaussian, and the Rayleigh length at the cavity mode focus is $1560 \mu \mathrm{m}$, which puts the depth of the cavity-mode focus larger than the dimension of the MOT. Based on this observation, the atom density is assumed to be uniform on the z-axis, and radially symmetric with the same Gaussian distribution on the $\mathrm{x}$ - and y-axes. Although the CCD camera only records the images on yz-plane, we can still reconstruct the volume density $n_{V}(x, y, z)$ with these symmetry conditions applied. The volume density distribution is expressed as,

$$
n_{V}(x, y, z)=N \times \frac{1}{L} \frac{1}{\sigma \sqrt{2 \pi}} \exp \left(\frac{-x^{2}}{2 \sigma^{2}}\right) \frac{1}{\sigma \sqrt{2 \pi}} \exp \left(\frac{-y^{2}}{2 \sigma^{2}}\right) .
$$




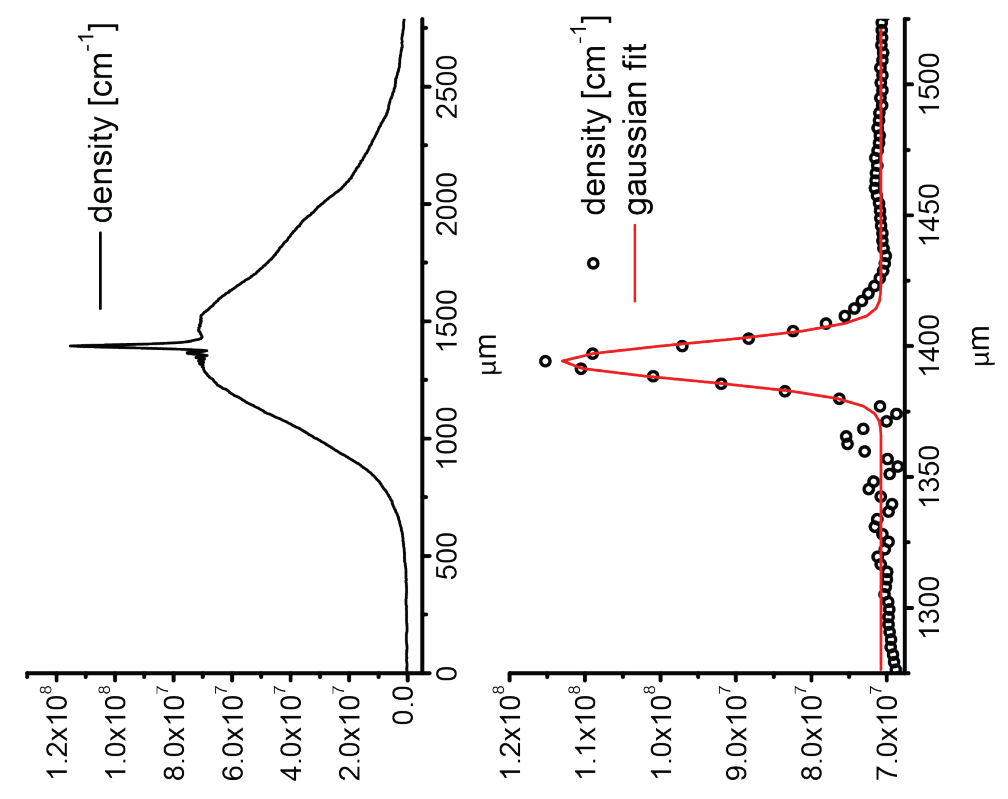

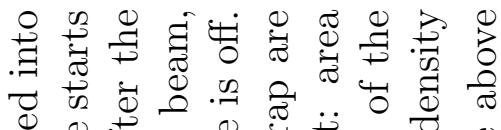

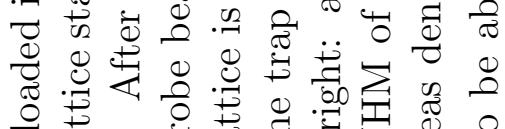

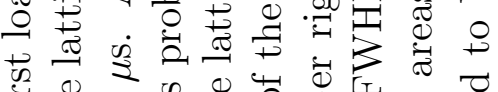

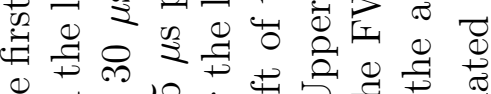

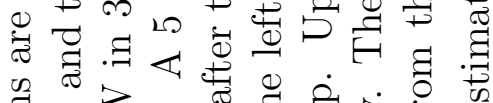

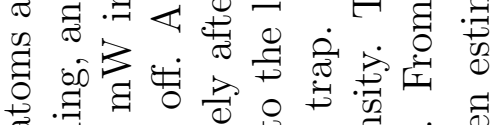
둥워

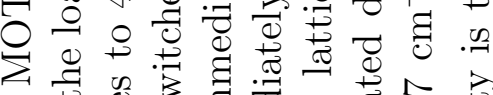

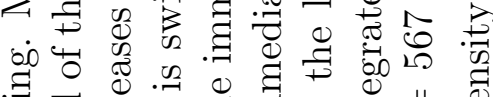

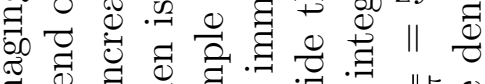

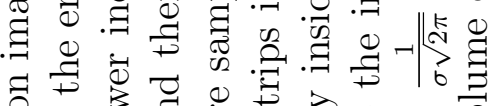

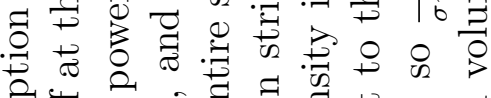

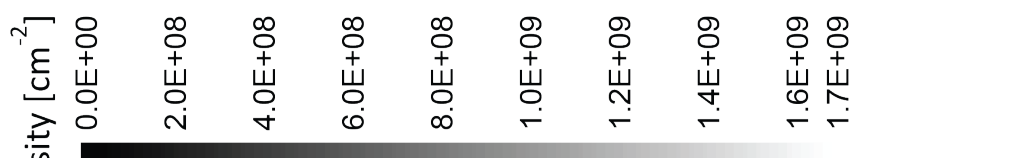

$\frac{1}{0}$

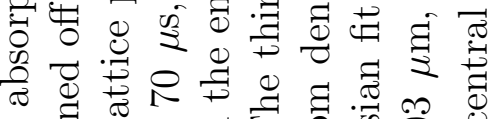
>

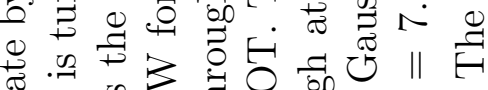

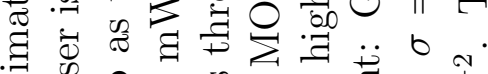

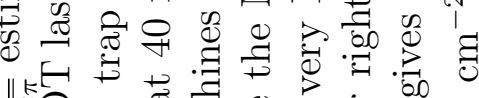

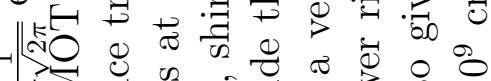

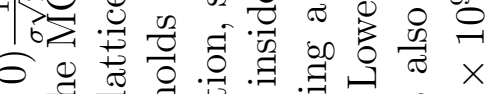

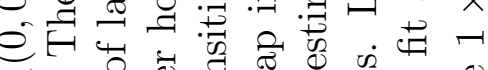

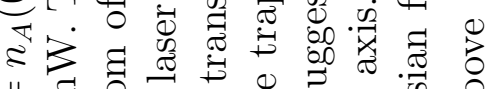

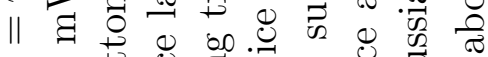

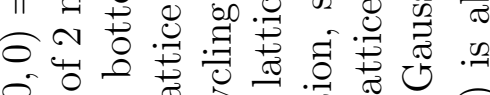

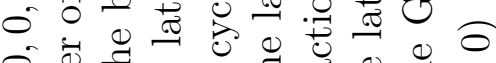

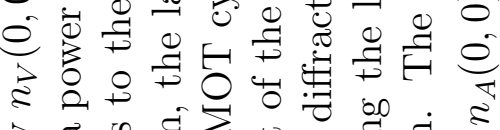

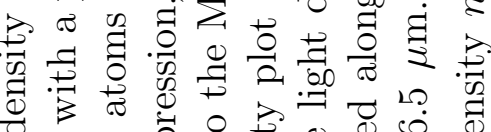

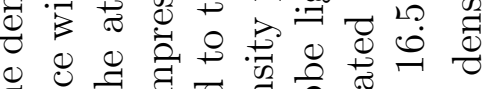
궁

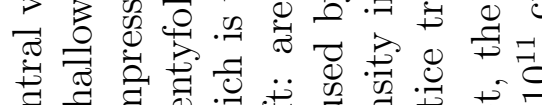

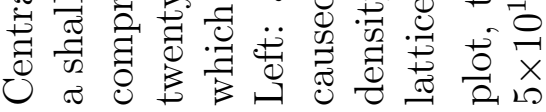

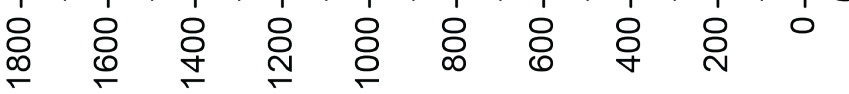

$\ddot{3}$
0
0
0
0
.0
.1 
where $N$ is the total number of atoms, and $L$ is the length of the lattice trap in the lattice axis. The CCD camera sees the area density on the yz-plane,

$$
n_{A}(y, z)=\frac{N}{L} \frac{1}{\sigma \sqrt{2 \pi}} \exp \left(\frac{-y^{2}}{2 \sigma^{2}}\right)
$$

Integrated the area density plot along lattice axis, or z-axis,

$$
\int_{-\frac{L}{2}}^{\frac{L}{2}} n_{A}(y, z) d z=\frac{N}{\sigma \sqrt{2 \pi}} \exp \frac{-y^{2}}{2 \sigma^{2}},
$$

$\sigma$ can be obtained by Gaussian-fitting the resulted curve, as shown in the right panel of Fig. 5.5. Since $n_{V}(x, y, z)=n_{A}(y, z) \frac{1}{\sigma \sqrt{2 \pi}} \exp \left(\frac{-x^{2}}{2 \sigma^{2}}\right)$, the central volume density follows,

$$
n_{V}(0,0,0)=n_{A}(0,0) \frac{1}{\sigma \sqrt{2 \pi}}
$$

where $n_{A}(0,0)$ is read directly from the center of the lattice area density plot. The volume density distribution is,

$$
n_{V}(x, y, z)=n_{V}(0,0,0) \exp \left(\frac{-x^{2}}{2 \sigma^{2}}\right) \exp \left(\frac{-y^{2}}{2 \sigma^{2}}\right)
$$

In the case of a lattice-compressed atom sample, the central volume density is usually higher than $2 \times 10^{11} \mathrm{~cm}^{-3}$.

The above calculation neglects the lattice potential wells in the z-axis and assumes a homogeneous distribution in this axis; the calculated density distribution is an average over z-axis. However, the actual density inside a single lattice potential well in the z-axis should be much higher than the density averaged over the z-axis. The atom number density depends how deeply the atom sample is compressed to the bottom of the lattice potential well. 


\subsection{Lattice adiabatic compression}

We can obtain a high $1064 \mathrm{~nm}$ lattice laser intensity at the center of the nearconcentric cavity. However, we were not certain whether very deep lattice traps can actually be loaded with MOT atoms. It was tested by both the earlier experiment in the group and my experiment that the lattice loading turns very inefficient when the lattice depth exceeds $50 \mathrm{MHz}$ or so.

In the case of a shallow lattice, the lattice-induced light shift is not so significant. MOT atoms keep filling the lattice, because they are still laser-cooled by the MOT cycling transition laser at the bottom of the lattice potential well. In the case of a deep lattice, the MOT light is so far-off-resonant at the bottom of the lattice potential well that the atoms are not laser-cooled there. Practically speaking, it is useless to turn up the lattice power more, because the MOT atoms stall at a depth of about $50 \mathrm{MHz}$. The number of atoms cooled to the bottom of the lattice trap and experiencing a large lattice-induced light shift might be so small that they are not experimentally detectable.

To overcome the lattice cooling limit, I adiabatically compress the atoms sample by ramping up the depth of the optical lattice. The idea of an adiabatic process is that if a Hamiltonian $H_{0}$ varies slowly and gradually, the nth eigenstate of $H_{0}$ will still be the nth eigenstate of the new Hamiltonian $H^{\prime}$. In the opposite case, if $H_{0}$ is changed all in a sudden (diabatic), then the nth eigenstate of $H_{0}$ will end up being a superposition of the eigenstates of the new Hamiltonian $H^{\prime}$.

As long as the lattice intensity increases slowly and gradually so the change is adiabatic, the atoms that are loaded to the bottom of a shallow lattice are expected to remain in the bottom of the new, deep optical lattice trap. The atoms are also expected to be more concentrated around the lattice axis, as the lattice potential becomes deeper and narrower. 


\subsubsection{Trap frequency of a deep optical lattice}

In the two-photon excitation spectroscopy, we usually use the lattice-induced light shift to describe the depth of the lattice. The other quantity that people often use to picture the geometry of an atomic trap is trap frequency: the optical potential seen by the atoms can be approximated by a simple harmonic potential, and the trap frequency is calculated from the "spring" constant related to this potential. For

example, the trap frequency is $f_{x}=\frac{\omega}{2 \pi}=\frac{1}{2 \pi} \sqrt{\frac{k_{x}}{m}}$ for a trap potential approximated by $V(x)=\frac{1}{2} k_{x} x^{2}$, and the atoms inside this potential oscillate at $f_{x}$. The trap frequency describes the time-scale of the motion of the atoms inside the trap.

For the case of my experiment, in the lattice axis, z-axis, the lattice optical potential is sinusoidal and periodic,

$$
V(z)=\frac{V_{0}}{2}(1+\cos 2 k z), \quad k=\frac{2 \pi}{\lambda}
$$

Using Taylor expansion, $V(z) \approx V_{0} k^{2} z^{2}$. The spring constant $k_{z}$ in the lattice-axis is $2 V_{0} k^{2}$. In the transverse direction, the lattice optical potential is exponential,

$$
V(\rho)=V_{0} \exp \left[\frac{-2 \rho^{2}}{w_{0}^{2}}\right]
$$

where $w_{0}$ is the beam waist of the lattice laser. Taking the second derivative, $V(\rho) \approx$ $\frac{2}{w_{0}^{2}} V_{0} \rho^{2}$. The spring constant $k_{\rho}$ in the transverse direction is $\frac{4 V_{0}}{w_{0}^{2}}$.

The optical potential amplitude $V_{0}$ is calculated from the a.c. Stark shift of the ground state, $V_{0}=-\frac{1}{4} \alpha_{5 \mathrm{~S}} E_{0}^{2}$, with $E_{0}^{2}=\frac{2 I}{c \epsilon_{0}}$. Using $\alpha_{5 \mathrm{~S}} \approx 700 \times 4 \pi \epsilon_{0} a_{0}^{3}, w_{0}=23 \mu \mathrm{m}$, $\lambda=1064 \mathrm{~nm}$, and a lattice laser intensity for the "shallow" lattice case $I=1.67 \times$ $10^{10} \mathrm{~W} / \mathrm{m}^{2}$ (corresponding to a transmitted lattice power about $2 \mathrm{~mW}$ ), the trap frequencies in the shallow lattice are: in the lattice axis $f_{z}=700 \mathrm{kHz}$, and in the transverse direction $f_{\rho}=7 \mathrm{kHz}$. 

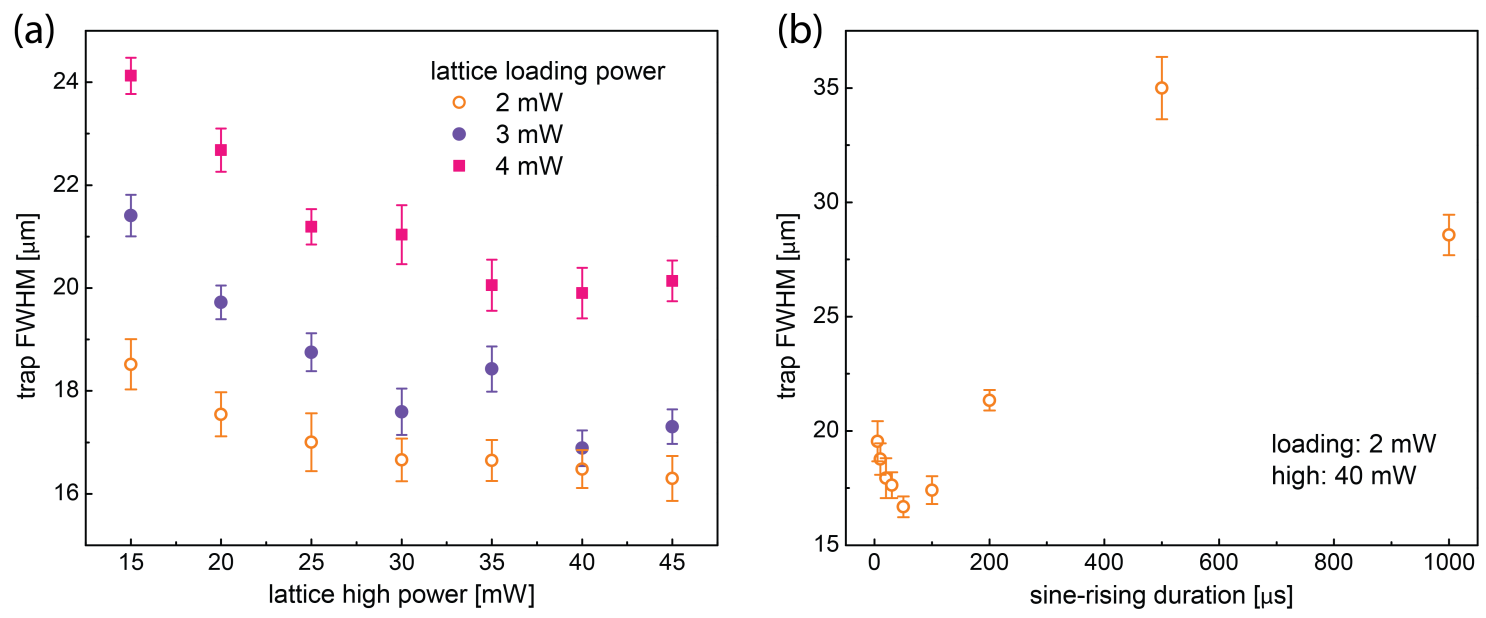

Figure 5.6:

FWHM of the atom density distribution in lattices of (a) different compression ratio (b) different sine-rising duration. The oscillation of FWHM after $100 \mu$ s might be due to the heating of the trapped atoms by the intense lattice laser field.

\subsubsection{Lattice intensity modulation}

To apply the adiabatic compression idea experimentally, the lattice is first loaded at a low power to have a large number of trapped atoms to start with. Then the MOT laser is switched off and the lattice power starts increasing. The lattice power follows a sine-rising curve (phase varies from $\frac{-\pi}{2}$ to $\frac{\pi}{2}$, Fig. 5.2 shows an example), so the increasing curve is smooth and slow. The atoms that were in the bottom of the shallow lattice trap are expected to remain in the bottom of the deep lattice despite the fact that the lattice power is increasing.

The best compression ratio (high power to loading power) and the sine-rising compression duration is determined experimentally by the methods of shadow imaging. The timing sequence of the shadow imaging is as follows: the lattice power is kept low and then sine increased to the high power. The lattice is held at high power for $70 \mu \mathrm{s}$ before being switched off of rapidly. A $5 \mu$ s probe tuned to the cycling transition is on while the lattice laser is off. Fig. 5.6(a) shows the trap FWHM in the shadow images at different compression ratios with the same compression duration of $30 \mu \mathrm{s}$. 
Given a fixed high power, the trap waist is generally the narrowest if the compression starts with $2 \mathrm{~mW}$, resulting in a higher compression ratio. Fig. 5.6(b) shows the dependence of trap FWHM on the compression duration when the lattice power is increased from $2 \mathrm{~mW}$ to $40 \mathrm{~mW}$. From the data we see the FWHM is the narrowest when the duration is about $50 \mu \mathrm{s}$, which corresponds to a sinusoidal modulation at $20 \mathrm{kHz}$. In the later spectroscopy experiments, we choose the lattice compression condition that gives a narrow FWHM, which implies a more spatially concentrated atom sample and less spectral line broadening.

The times scale of the lattice intensity modulation is about $20 \mathrm{kHz}$. The initial trap frequencies are $f_{z}=700 \mathrm{kHz}$, and $f_{\rho}=7 \mathrm{kHz}$. Based on the frequencies, we expect that the atoms get adiabatically compressed in the z-axis. We are not certain about the transverse direction, because the initial trap frequency is less than the intensity modulation frequency. Since the trap frequencies are proportional to the depth of the lattice potential, we expect the compression to be more and more adiabatic as the lattice increased to higher power. Overall, the atom sample after the lattice compression might be a product of a mixture of adiabatic and diabatic compression, but more likely to be adiabatic. In comparison, if we had utilized an optical dipole trap made of a single focused beam without periodic optical lattice modulation along the beam axis, then the trap frequencies would have been $f_{\rho}=7 \mathrm{kHz} \gg f_{z}$, due to the large Rayleigh length. The lattice compression would have to be carried out much more slowly to ensure an adiabatic compression, with the price of slowing the data-taking rate and losing more atoms during the long compression duration.

\subsubsection{Two-photon excitation spectroscopy}

The atom density estimation by the shadow imaging method indicates a significant increase of the atoms density in a lattice-compressed sample. However, we are not certain whether the atoms are adiabatically compressed to the bottom of the trap 

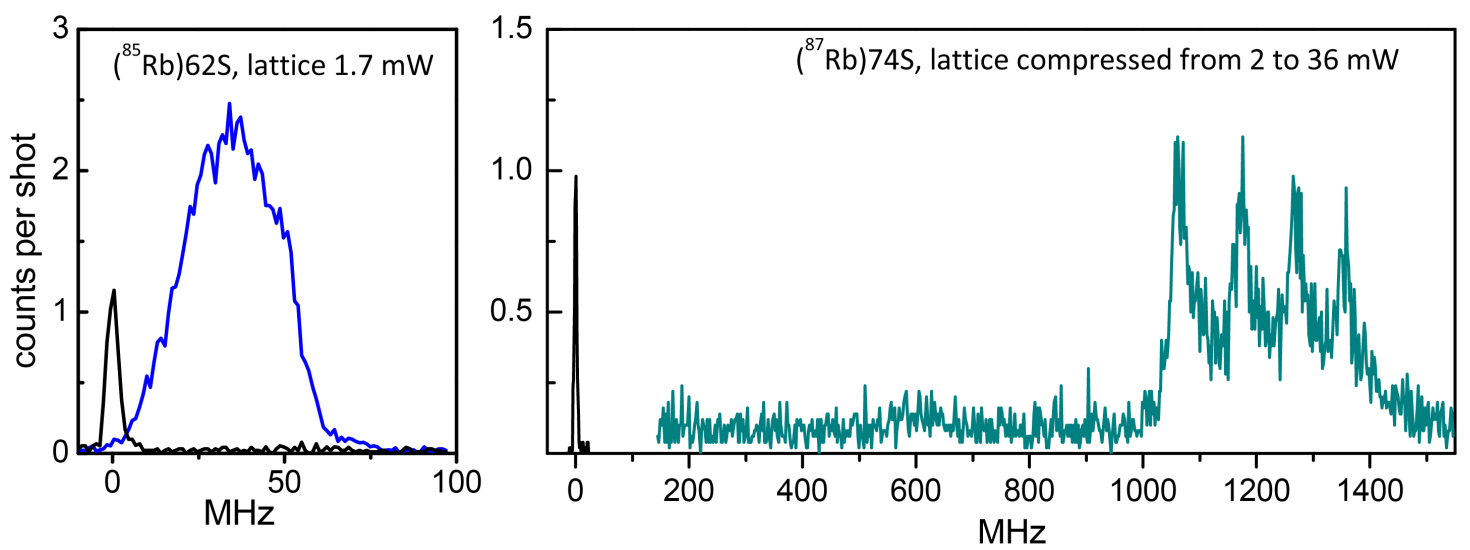

Figure 5.7:

Two-photon excitation spectra. (a) Lattice is always at loading power. (b) Lattice is at loading power for most of the time, but compressed to high power immediately before the Rydberg excitation. The splitting of $74 \mathrm{~S}$ signal is due to the intermediate state $5 \mathrm{P}_{3 / 2}$.

due to the limited resolution of the cameras. In comparison, the effect of adiabatic compression is transparent in two-photon excitation spectra.

Fig. 5.7 shows spectra of a shallow lattice and an adiabatically-compressed lattice. The narrow lines on the spectra are from off-resonance excitation of MOT atoms outside the lattice. The blue-shifted, broadened signals are from the atoms inside the lattice. For the shallow lattice, the lattice signal is very close to the MOT signal, and the sharp cut-off of the broadened lattice signal at the blue side typically ends somewhere around $50 \mathrm{MHz}$ (for a lattice power $\gtrsim 2 \mathrm{~mW}$ ). For the case of an adiabatically compressed sample, the lattice signal is far away from the MOT signal, and in-between the two signals there is a wide void region. This spectral feature suggests that the atoms that were at the bottom of the shallow lattice remain at the bottom of the deep lattice after the adiabatic compression is done.

With the lattice adiabatic compression, we obtain a fairly large population of atoms at the bottom of a very deep optical lattice trap, with lattice-induced light shifts on the order of several GHz. The lattice adiabatic compression also helps to reduce the broadening of the lattice-shifted spectral signals in the two-photon 
excitation spectroscopy, because the atoms are more concentrated around the lattice axis. There are several immediate applications of the lattice adiabatic compression, which are discussed in the following chapters, including radiation guiding (Chap. VI), polarizability measurement (Chap. VII) and Rydberg hydrogenic states ( Chap. VIII). 


\section{CHAPTER VI}

\section{Radiation guiding}

In Sec. 4.2, I showed the results of the radiation guiding in a shallow lattice trap, and concluded that the elongated cold-atom cloud acts like a "mini-fiber". After upgrading the cavity-stabilization scheme, I am able to "lattice-compress" the coldatom samples and switch the lattice off for observation. In the is chapter, I present the more prominent radiation guiding phenomena in the deep optical-lattice traps and a rough model that qualitatively explains what I observed. In the following discussion, I use the $\mathrm{HG}_{00}$ cavity mode, the fundamental Gaussian cavity mode, which is also the easiest to align.

\subsection{Imaging system}

The radiation guiding by the lattice-compressed cold-atom sample is monitored by the "top camera", which looks though the cavity mirror from above. Imaging the cold-atom cloud at the trap center is made possible by two features of the experimental setup: First, the cavity mirror is approximately transparent to the $780 \mathrm{~nm}$ laser light (transmission of 80\%). Second, the cavity is of near-concentric configuration. The fluorescence or probe light scattered by the atoms at the cavity center is emitted outward in all directions. As a result, that the $780 \mathrm{~nm}$ fluorescence/scattered probe light comes out from a point source which is located directly at the center of a 


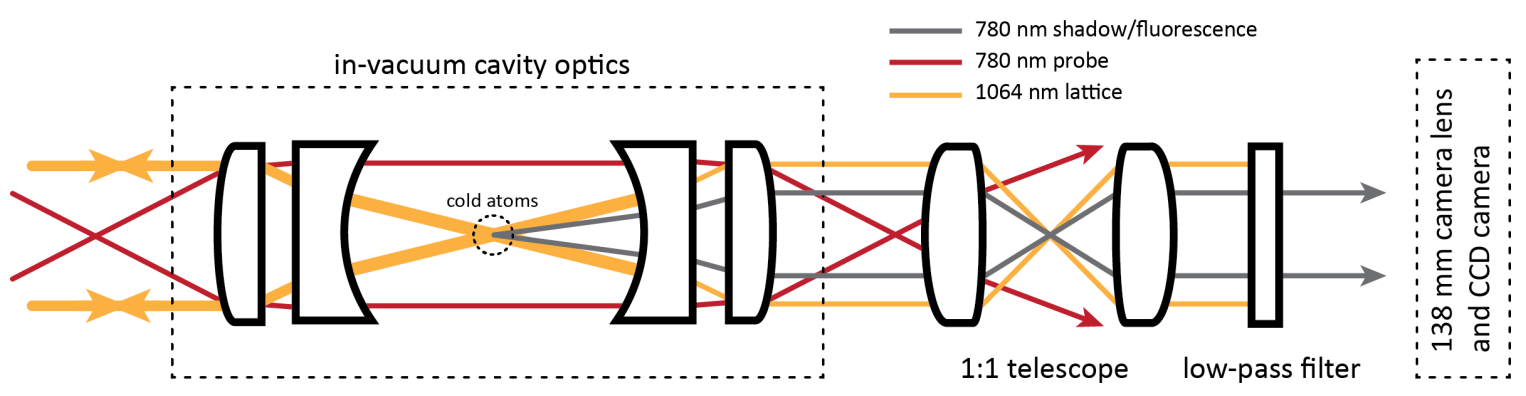

Figure 6.1:

Illustration of the laser light path in the shadow/fluorescence imaging. Not shown in the plot: a long pass dichroic mirror that separates the $780 \mathrm{~nm}$ and $1064 \mathrm{~nm}$ laser light before the lowpass filter.

concentric cavity. The propagation directions of the light rays starting from this point source are distributed in the light cone defined by the cavity mirror of the cavity at the exit port. The solid angle of the light cone is about $0.058 \pi$, which is limited by the inner radius of the ring piezo between the cavity mirror and the coupling lens. These rays are collimated by the cavity mirror and the coupling lens in the same way that the transmitted lattice laser is collimated.

Fig. 6.1 illustrates the imaging mechanism. The fluorescence or the shadow caused by the probe light scattered by the atoms are imaged on the camera in the same manner. In the case of shadow imaging, I apply a probe laser beam (typically $5 \mu$ s, $10 I_{\text {sat }}$ ), which is divergent before entering the cavity in a way that it is approximately collimated at the trap center. A 1:1 telescope after the cavity helps correct the collimation of the fluorescence/shadow imaging light coming out from the cavity center. Then, the fluorescence/shadow is focused on the CCD detector of the top camera by a $138 \mathrm{~mm}$ camera lens. The probe light is not collimated after the cavity, so it is not imaged sharply on the camera; it forms an approximately homogeneous background.

The optical imaging system for obtaining fluorescence and shadow images is the same, the difference is in the operating mode of the camera. In the case of fluorescence imaging, the camera is not triggered and has a very long exposure time (50 ms) due 
to the low intensity of the fluorescence. In the case of shadow imaging, the camera is triggered on when the probe pulse is on (intensity $\approx 10 I_{\text {sat }}$ ), and has a short exposure time $(300 \mu \mathrm{s})$.

\subsubsection{Radiation guiding as an experimental alignment tool in Rydberg atom spectroscopy}

In the two-photon Rydberg excitation, the lower transition laser, the $780 \mathrm{~nm}$ laser which drives the same $5 \mathrm{~S}_{1 / 2} \rightarrow 5 \mathrm{P}_{3 / 2}$ transition as in the case of the shadow imaging, is focused at the trapped center. I use a pair of counter-propagating $780 \mathrm{~nm}$ lower transition lasers to reduce the radiation pressure caused by the $780 \mathrm{~nm}$ laser intensity. The $780 \mathrm{~nm}$ laser beams are set through the cavity mirrors: One co-propagates with the lattice laser input, and enters the cavity through the cavity mirror at the input side. The other one counter-propagates with the lattice laser and enters the cavity through the cavity mirror at the exit port. By looking directly through the cavity mirrors with the top camera when the lattice laser is locked to a low power $(\approx 3 \mathrm{~mW})$, I can easily overlap the focus of the co-propagating lower transition laser with the elongated cold-atom cloud. The rough alignment is done as follows: first, I use fluorescence imaging to determine the location of the trap center on the camera's real-time monitoring window, as shown in the screen shots in Fig. 4.2 or 4.3 . Second, I move the direction of the $780 \mathrm{~nm}$ laser beam, so that it overlaps the position of the trap center from the view of the top camera. The overlap is further optimized by maximizing the absorption of the lower transition laser: I trigger the top camera so that the camera only sees the short pulse of the $780 \mathrm{~nm}$ laser light but no MOT fluorescence, and then I tweak the direction of the $780 \mathrm{~nm}$ laser beam slightly such that it transmission through the sample decreases when its frequency sweeps over the $5 \mathrm{~S}_{1 / 2} \rightarrow 5 \mathrm{P}_{3 / 2}$ cycling transition. Fig. 6.2 shows screen shots of the lower transition laser beam as its frequency sweeps over the $5 \mathrm{~S}_{1 / 2} \rightarrow 5 \mathrm{P}_{3 / 2}$ transition. The overlap of 


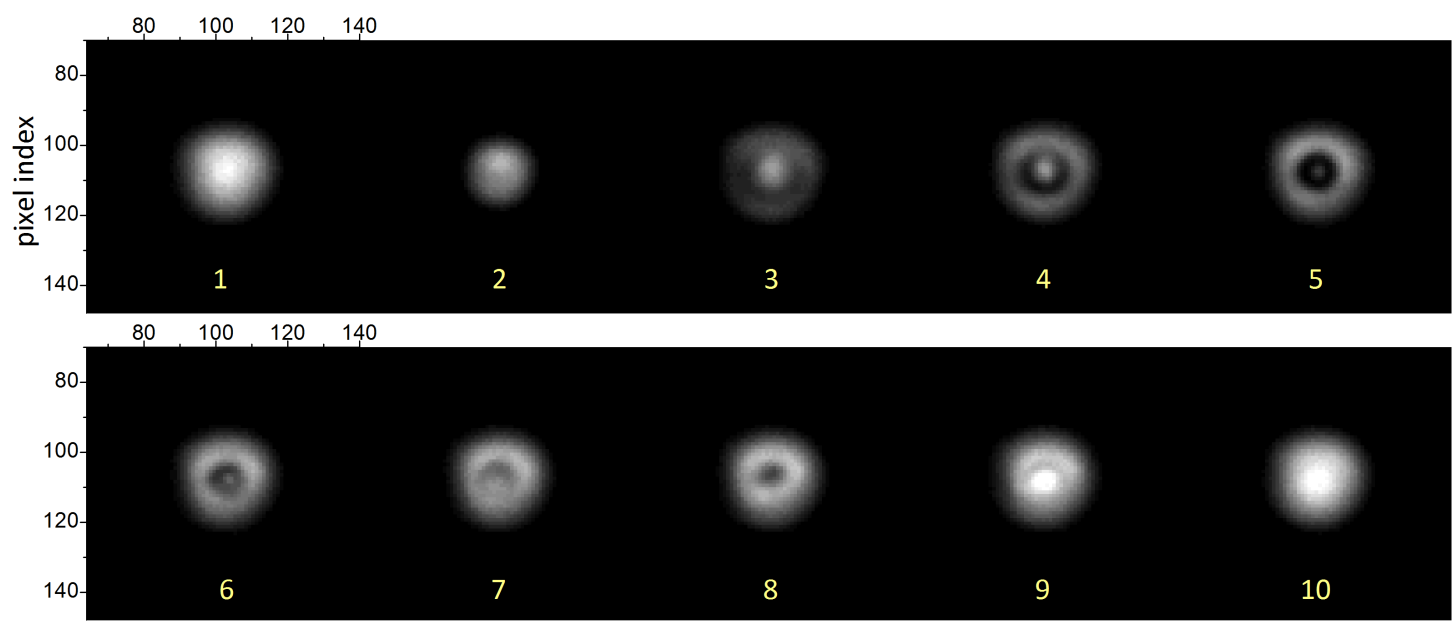

Figure 6.2:

Lower transition laser lensing by the atoms in a shallow lattice trap. The lower transition laser is focused in, and its waist $(50 \mu \mathrm{m})$ is about twice of the waist of the cavity mode $(23 \mu \mathrm{m})$. The number is the order of the screenshots, as the lower transition frequency sweep through the cycling transition frequency.

the lower-transition beam with the lattice trap is optimized such that I observe the concentric diffraction ring patterns.

Both of the lower transition lasers are brought to the experimental setup by singlemode fibers. After the one that co-propagates with the lattice laser is optimized by the method of radiation guiding, the counter-propagating lower-transition beam laser is then overlapped by back-coupling it into the fiber that sends out the first lowertransition laser beam.

\subsection{Radiation guiding}

\subsubsection{Timing sequence}

The experimental repetition rate for the shadow imaging used to probe the latticecompressed atom sample is $20 \mathrm{~Hz}$. Most of the time during the experimental cycle, the lattice is held at the loading power to load the MOT atoms, except at the end 


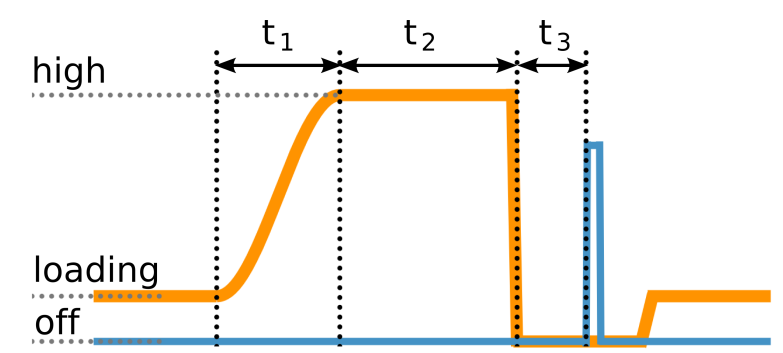

Figure 6.3:

Illustration of the timing sequence. Orange: lattice laser power. Blue: probe pulse. $t_{1}$ : compression duration. $t_{2}$ : hold high. $t_{3}$ : probe pulse delay.

of the cycle. The timing sequence at the end of the experimental cycle is shown in Fig. 6.3. The lattice compresses the atom sample with a compression duration of $t_{1}=50 \mu \mathrm{s}$, and then the lattice is held at high power for $70 \mu \mathrm{s}$. It is then finally switched off for the observation with a probe pulse that is on-resonant with the MOT cycling transition $\left(5 \mathrm{~S}_{1 / 2} \mathrm{~F}=2 \rightarrow 5 \mathrm{P}_{3 / 2} \mathrm{~F}^{\prime}=3\right)$. The probe beam is pulsed on for $5 \mu \mathrm{s}$ when the lattice is off, and the intensity is on the order of $10 I_{\text {sat }}$. The relatively high probe intensity is necessary due to the very strong attenuation by the elongated cold atom cloud. The MOT light is switched off before the lattice starts compression, and is kept off during the probe observation.

Fig. 6.4 shows the time-delayed shadow images of a lattice-compressed ${ }^{87} \mathrm{Rb}$ atom sample. The lattice compresses from $2 \mathrm{~mW}$ to $50 \mathrm{~mW}$, and the $5 \mu$ s probe pulse is on after different delay times $\left(t_{3}\right.$ in Fig. 6.3). The attenuation at the trap center is so strong that the probe light is completely extinguished there; we are not able to do an atom density measurement from the shadow image. However, by comparing the size of the dark disk of different time-delayed images, we can tell how much the atom sample has spread out during the delay of the probe pulse. As shown in the figure, the size of the dark disk is nearly the same between no delay and a $10 \mu$ s delay. As a result, even though the lattice trap is switched off for observation, as long as the $5 \mu \mathrm{s}$ probe beam is immediately on after the lattice is off, we assume that the atom 


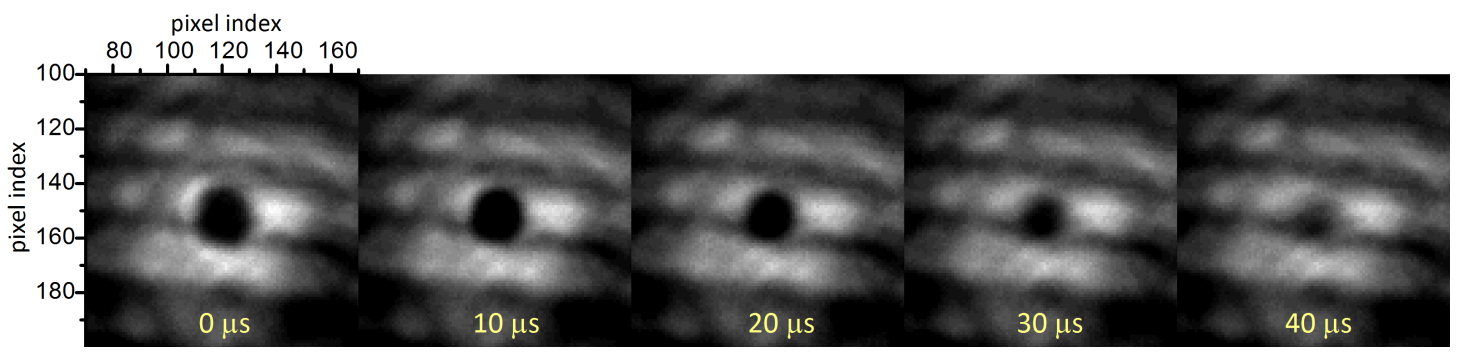

Figure 6.4:

Shadow images of a lattice compressed atom sample at various probe pulse delays. The bright background is the probe light field, which is banded because of interference effects caused by the vacuum window or other optics components along the beam path. The dark disk at the center is the shadow of the lattice-compressed atom sample, and it is usually completely saturated. 1 pixel $=2.45 \mu \mathrm{m}$ in the object plane.

sample remains the same during the observation.

Also in Fig. 6.4, the dark disk becomes smaller after a $20 \mu$ s delay. This is likely due to the thermal expansion of the atomic cloud in the transverse direction. The lattice compression increases the temperature of the atoms in the lattice by a factor of about $\frac{I}{I_{0}}$, where $I$ is the final and $I_{0}$ is the initial lattice laser intensity. As a result, in Fig. 6.3, the atom temperature is increased by a factor of $\frac{50}{2}=5$ after the compression. The initial temperature of the (MOT-cooled) atoms is about $200 \mu \mathrm{K}$. After the compression, the atom temperature is about $1 \mathrm{mK}$, which corresponds to a speed of $0.3 \mathrm{~m} / \mathrm{s}$; the atoms could move outward by a distance of $3 \mu \mathrm{m}$ every $10 \mu \mathrm{s}$.

\subsubsection{Lensing effect}

Fig. 6.5 shows the shadow images and vertical cuts of a lattice-compressed ${ }^{87} \mathrm{Rb}$ sample with probe laser at different detunings. A $2 \mathrm{~mW}$ lattice is first loaded with MOT atoms, the loaded sample is then compressed by increasing the lattice laser power to $40 \mathrm{~mW}$ in $50 \mu \mathrm{s}$. The probe frequency is detuned relative to the MOT cycling transition, $5 \mathrm{~S}_{1 / 2}(F=2) \rightarrow 5 \mathrm{P}_{3 / 2}\left(\mathrm{~F}^{\prime}=3\right)$. The brightness of the images is enhanced to make the features in the images visible. The location of the vertical cut is indicated by the yellow markers. The cut shows pixel values relative to the probe 
background field when there is no atom sample. The trap center is indicated by the dashed line on the plots. In the region away from the trap center, the relative pixel values approximately cancel to zero, except in the case of a $-2.4 \mathrm{MHz}$ probe detuning, where the probe is nearly on-resonance with the MOT cycling transition, leading to absorption by the atoms outside the lattice trap.

In the following, I give a brief description of each image and its vertical cut.

- -33.9 MHz. There is a strong emission coming out from the dark disk at the trap center, plus an inner bright rim and an outer dark rim circling the trap.

- $-11.5 \mathrm{MHz}$. The dark disk becomes larger, which suggests a stronger attenuation because of a smaller probe detuning. There is no emission from the trap center, but we can see the inner bright rim and the outer dark rim that circle the trap.

- $-2.4 \mathrm{MHz}$. The probe frequency is close enough to the $5 \mathrm{~S}_{1 / 2}(\mathrm{~F}=2) \rightarrow 5 \mathrm{P}_{3 / 2}\left(\mathrm{~F}^{\prime}=\right.$ 3) transition, so it experiences a strong attenuation at the trap center, and attenuation outside the trap due to the less dense non-trapped atom cloud. There is no rim pattern circling the trap.

- $10.9 \mathrm{MHz}$. The size of the dark disk is about twice as small as for the case of the red-detuned probe. Concentric inner bright and the outer dark rims appear again.

- 43.0 MHz. The attenuation of the probe light is not obvious. Instead, there is enhanced intensity at the trap center. We can observe a faint pattern consisting of an inner bright rim and an outer dark rim.

From the images, we see two different types of lensing effects: 1 : Center guiding of the probe light, seen as bright emission from the trap center, and surface guiding,

\footnotetext{
${ }^{1}$ In order to see the effect as shown in Fig. 6.5, one has to carefully couple the probe light into the cold-atom "mini-fiber". If the probe enters the trap at a small angle other than co-propagating with the lattice laser, the emission, both with red-detuned or blue-detuned probe, would then emerges as a single bright spot next to the dark disk of the trap instead of forming concentric patterns.
} 


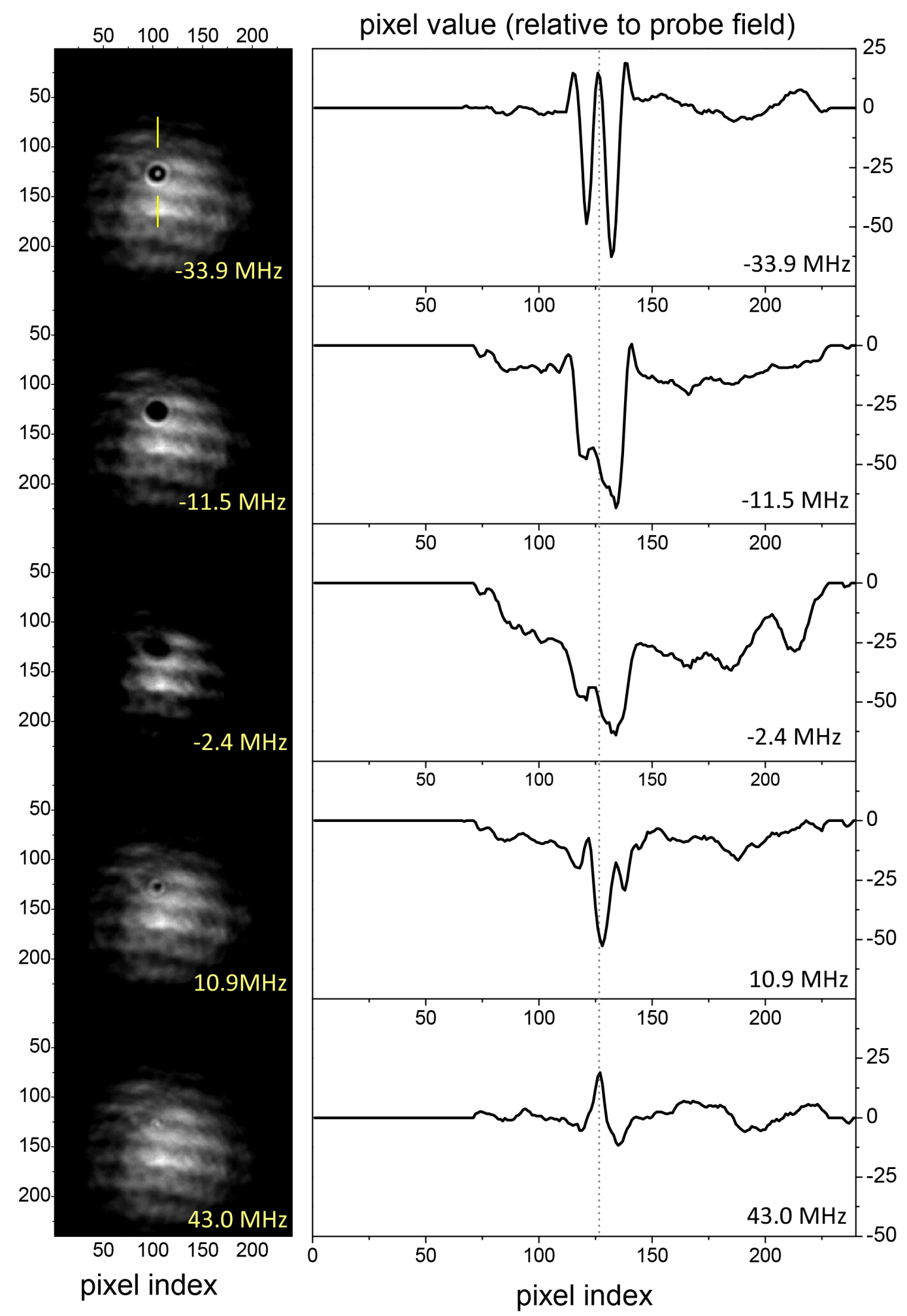

Figure 6.5:

Radiation guiding in a lattice-compressed ${ }^{87} \mathrm{Rb}$ sample. The uncertainty of the probe frequency is $\pm 1 \mathrm{MHz} .1$ pixel $=2.45 \mu \mathrm{m}$ in the object plane. 


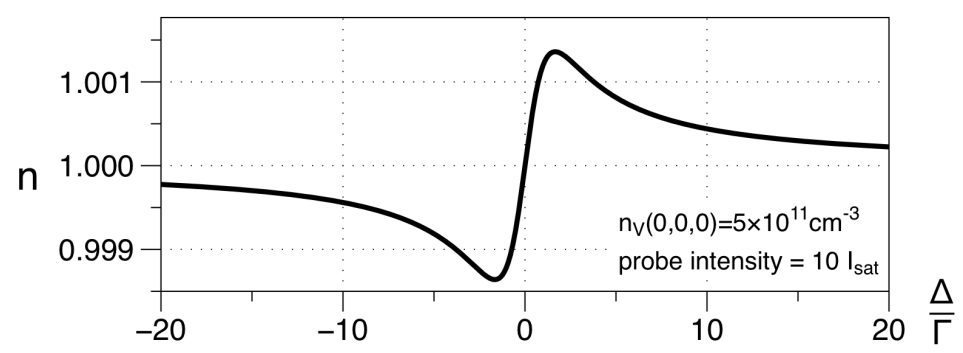

Figure 6.6: Dispersion curve, using Eq. 6.2

seen as a bright rim circling the trap. The multiple dark/bright rim patterns might be caused by a specific diffraction behavior of the probe wavefront as it propagates through the atom cloud.

\subsection{Qualitative model}

We use a two-level system to describe the lensing effect by the dense, elongated atom cloud: $5 \mathrm{~S}_{1 / 2}$ is the ground state, level 1 , and $5 \mathrm{P}_{3 / 2}$ is the excited state, level 2 . The probe light drives the $5 \mathrm{~S}_{1 / 2}(\mathrm{~F}=2) \rightarrow 5 \mathrm{P}_{3 / 2}\left(\mathrm{~F}^{\prime}=3\right)$ transition, and its intensity is on the order of $10 I_{\text {sat }}$.

Assuming there is saturation due to the probe intensity, at steady state, the coherence $\tilde{\rho}_{12}$ is,

$$
\begin{aligned}
\operatorname{Re}\left[\tilde{\rho}_{12}\right] & =\sqrt{\frac{I}{2 I_{\text {sat }}}} \frac{\frac{2 \Delta}{\Gamma}+4\left(\frac{\Delta}{\Gamma}\right)^{2}+\frac{I}{I_{\text {sat }}}}{\operatorname{Im}\left[\tilde{\rho}_{12}\right]}=\sqrt{\frac{I}{2 I_{\text {sat }}}} \frac{1}{1+4\left(\frac{\Delta}{\Gamma}\right)^{2}+\frac{I}{I_{\text {sat }}}}
\end{aligned}
$$

where $\Gamma$ is the decay rate in $\mathrm{MHz}\left(6.0666 \mathrm{MHz}\right.$ for $5 \mathrm{~S}_{1 / 2} \rightarrow 5 \mathrm{P}_{3 / 2}$, and $\Delta$ is the probe detuning in $\mathrm{MHz}$. The absorption coefficient $\left(\mathrm{m}^{-1}\right)$ and index of refraction (unit-less) 

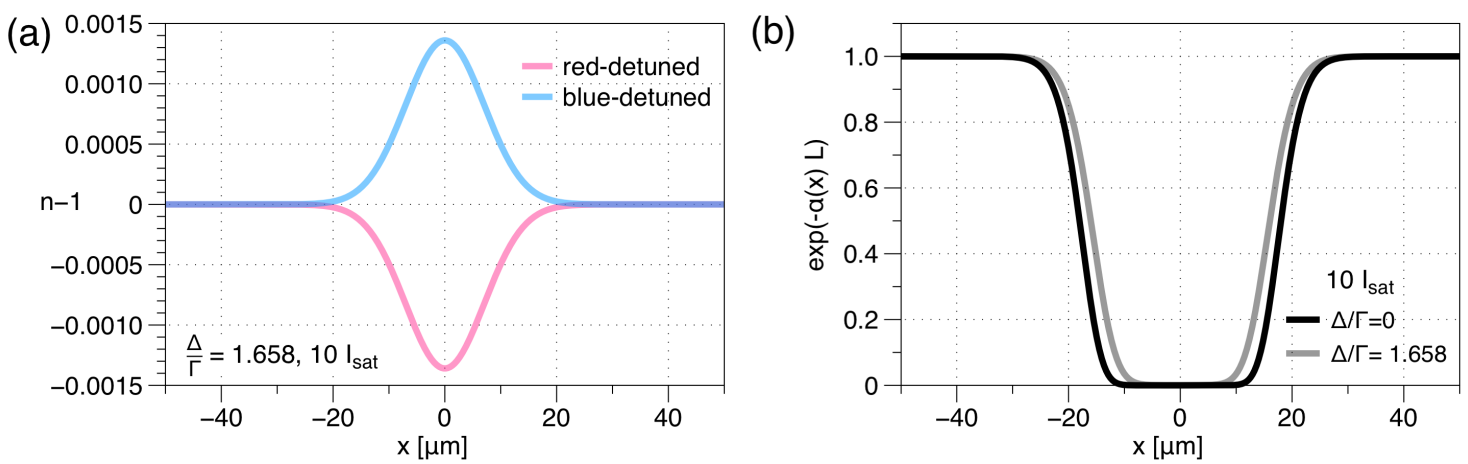

Figure 6.7:

Index of refraction and the absorption in the transverse direction, using Eq. 6.2

depend on the coherence,

$$
\begin{aligned}
n-1 & =\frac{1}{2} \frac{2 \mathrm{n}_{\mathrm{v}} d}{\epsilon_{0} E_{0}} \operatorname{Re}\left[\tilde{\rho}_{12}\right] \\
\alpha & =\frac{2 \mathrm{n}_{\mathrm{v}} d \omega}{c \epsilon_{0} E_{0}} \operatorname{Im}\left[\tilde{\rho}_{12}\right]
\end{aligned}
$$

where $d$ is the dipole matrix element of the transition, $\omega$ is the angular transition frequency, and $n_{V}$ is the atomic number volume density. $n_{V}$ is cylindrically symmetric about the z-axis in my experiment, as discussed in Sec. 5.2. I use the experimental parameter from Fig. 5.5 to plot the dispersion relation in Fig. 6.6. trap $\sigma=7.03 \mu \mathrm{m}$, trap length along the lattice axis $L=1400 \mu \mathrm{m}$, and a central volume density $\mathrm{n}_{\mathrm{V}}(0,0,0)=5 \times 10^{11} \mathrm{~cm}^{-3}$. From the dispersion curve, the maximum and minimum of $n$ occurs at $\frac{\Delta}{\Gamma}= \pm 1.66$, or $\pm 10 \mathrm{MHz}$. I use $\frac{\Delta}{\Gamma}= \pm 1.66$ to plot the index of refraction and the absorption along the x-axis in Fig. 6.7.

Because of the Gaussian profile and the sign of the index of refraction, we expect a blue-detuned probe will be diverging when passing through the atom cloud. Whereas the red-detuned probe will be converging. However, we are not entirely certain about the way the probe light emerges from the trap, because of the strong absorption by the trap. To give a qualitatively description, we assume the probe light field is a plane wave, and we plot the wavefront of the probe light after it passes through the 
trap, using the Raman-Nath approximation. I.e., we assume there is no change of the wavefront in the transverse direction as it propagates in the elongated cold-atom cloud, and the probe wavefront only accumulates a phase change in the direction of propagation. The phase change is,

$$
\Delta \phi=\frac{2 \pi}{\lambda}[n(x)-1] L
$$

Fig 6.8 shows the plot of the phase of the probe light wavefront and the intensity after the probe passing though the atom cloud. ${ }^{2}$ From the phase of the wavefront of the probe, we expect a red-detuned probe light forms one focus along the z-axis, whereas we expect a focal ring in the blue-detuned case. Also, because of the radius of curvature of the wavefront, the focal length of the blue-detuned focal ring is larger than of the red-detuned focus. We plot the intensity of the probe light after the atom cloud, using the absorption coefficient in Eq. 6.2. For the red-detuned probe, the calculated radius of curvature of the wavefront at $z=0$ is $26 \mu \mathrm{m}$. We use this distance as the focal length and plot the intensity in the imaging plane that contains the focus. The imaging plane of the blue-detuned probe on the plot is twice as far as that of the red-detuned. Although there is strong absorption by the atom cloud, qualitatively, the probe light has emission coming out from the trap center in the red-detuned case, and there is a bright rim around the trap in the blue-detuned case.

The above analysis does not explain the multiple rim pattern and the very strong emission from the trap center. For a more complete model, one should solve the Helmholtz equation for the probe wave propagating in the elongated cold-atom cloud. This suggested approach is based on the observation that the elongated cold-atom cloud is similar to a gradient-index rod whose index of refraction gradually varies in

\footnotetext{
${ }^{2}$ I use the probe wavefront emerging from the cold-atom could to plot the intensity in the image plane. I assume that at a location $\mathrm{x}$ in the one-dimensional wavefront, the light rays start from this $\mathrm{x}$ location and propagate in the normal direction of the wavefront, and the number of light rays is proportional to $\mathrm{e}^{-\alpha(x) L}$. The intensity in the image plane is plotted as a histogram of the number of light rays that reach the bin at the corresponding $\mathrm{x}$ position in image plane.
} 

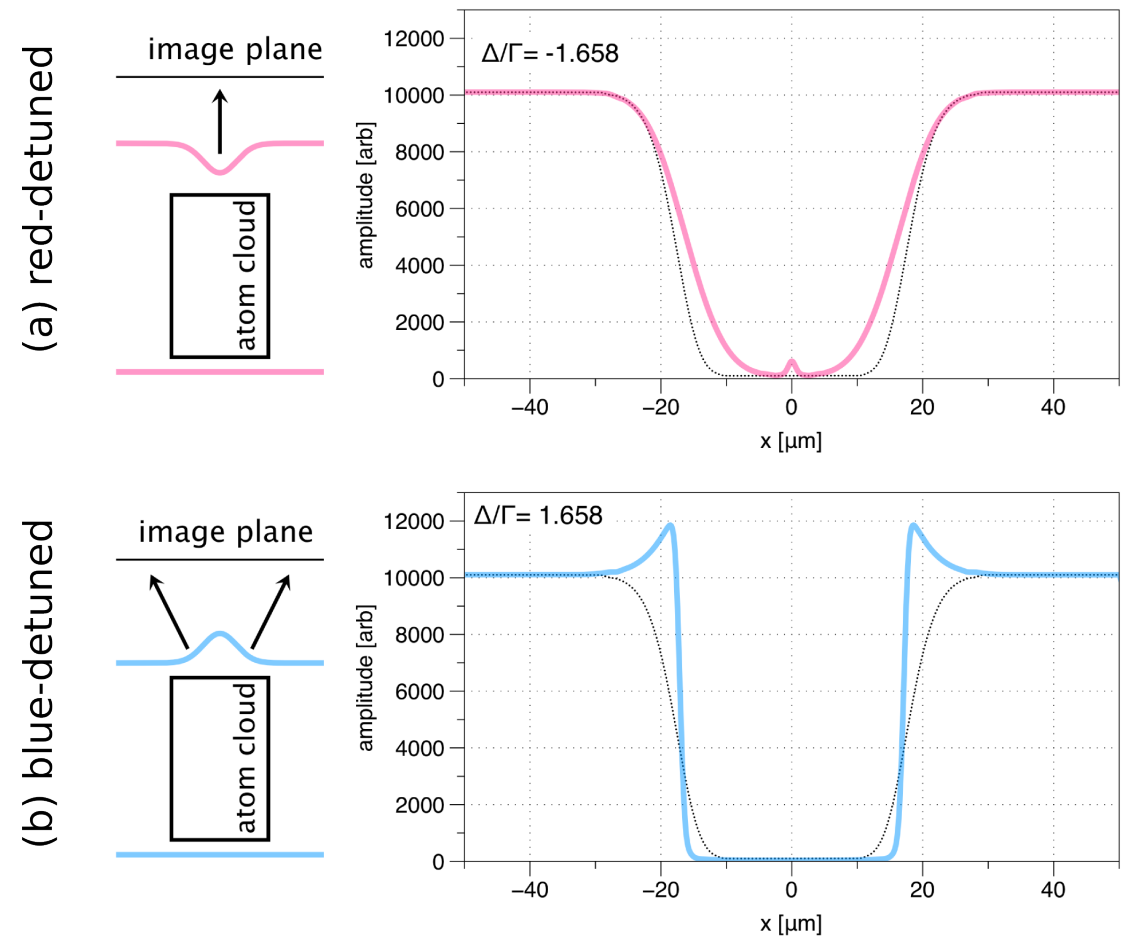

Figure 6.8:

Probe light wavefront phase and amplitude after passing the trap. (a) red-detuned probe. (b) blue-detuned probe. The dotted line in both plot is the amplitude of the on-resonance probe.

the radial direction. Nevertheless, the trap is not only good for making cold-atom mini-fiber, but also good for other experimental applications, such as Rydberg EIT [43, 44] or Rydberg polariton [45] experiments, in both cases the large aspect ratio $(\approx 100)$ and the high atom density of the trap will be great advantages. 


\section{CHAPTER VII}

\section{Dynamic polarizability measurement}

In this chapter, I discuss the experimental measurement of the dynamic polarizabilities of rubidium $5 \mathrm{P}_{3 / 2}$ in linearly-polarized $1064 \mathrm{~nm}$ lattices.

\subsection{Introduction - Stark shift and dynamic polarizability}

In the presence of an external static electric field, a neutral atom is polarized by the field, and thus its atomic energy levels are altered. This phenomenon is known as d.c. Stark shift. The interaction Hamiltonian of the electric field is,

$$
\hat{H}_{\mathrm{E}}=-\frac{1}{2}\left[\alpha_{0}+\alpha_{2} \frac{3 J_{z}^{2}-J(J+1)}{J(2 J-1)}\right] E_{0}^{2} \quad \text { (d.c. field) }
$$

where $\mathbf{E}=E_{0} \hat{\mathbf{z}}$ is the external electric field. The term inside the bracket is the polarizability of the atomic level, with the quantum number of the total angular momentum $J . \alpha_{0}$ is called scalar polarizability, and $\alpha_{2}$ is called tensor polarizability.

In case of an a.c. field, the interaction Hamiltonian is multiplied by $\frac{1}{2}$ to account for the electric field amplitude averaging $\left(E_{\mathrm{rms}}=\frac{1}{\sqrt{2}} E_{0}\right)$. Assuming the external electric field is linearly-polarized along the z-axis with field amplitude $E_{0}$, then the interaction Hamiltonian is, 


$$
\hat{H}_{\mathrm{E}}=-\frac{1}{4}\left[\alpha_{0}(\omega)+\alpha_{2}(\omega) \frac{3 J_{z}^{2}-J(J+1)}{J(2 J-1)}\right] E_{0}^{2} \quad \text { (a.c. field) }
$$

where $\alpha_{0}(\omega)$ and $\alpha_{2}(\omega)$ are now frequency-dependent a.c. polarizabilities.

In the experiment, we use $1064 \mathrm{~nm}$ lattice to trap rubidium atoms. The lattice laser intensity is converted into the amplitude of electric field by,

$$
I_{0}=\frac{1}{2} c \epsilon_{0} E_{0}^{2}
$$

The atomic levels that are involved in the lattice trapping are $5 \mathrm{~S}_{1 / 2}$ and $5 \mathrm{P}_{3 / 2}$. The dynamic polarizability of the ground-state rubidium $5 \mathrm{~S}_{1 / 2}$ depends only on $\alpha_{0}$, and $\alpha_{2}$ is zero (because $J=\frac{1}{2}$ ). For the case of $5 \mathrm{P}_{3 / 2}$, both $\alpha_{0}$ and $\alpha_{2}$ contribute to the polarizability.

In the following discussion, we assume that the equilibrium of the polarization process is fast-established when the lattice field is changing, and we neglect all the transient effects. This means that adiabatic following applies, i.e., the electric dipole is a well-defined function of the lattice electric field, and is always well characterized by that field. In a linear-polarization regime, the dipole is proportional to the field $(\mathbf{p}=\alpha \mathbf{E})$.

The characterization of the cavity-generated optical-lattice-induced light shift of the atomic energy levels requires the diagonalization of the full Hamiltonian in various field strength regimes. The full Hamiltonian for the atom inside an a.c. field is $\hat{H}=\hat{H}_{\mathrm{HFS}}+\hat{H}_{\mathrm{E}} \cdot \hat{H}_{\mathrm{HFS}}$ is the Hamiltonian of the hyperfine structure,

$$
\hat{H}_{\mathrm{HFS}}=A_{\mathrm{HFS}} \hat{\mathbf{I}} \cdot \hat{\mathbf{J}}+B_{\mathrm{HFS}} \frac{3(\hat{\mathbf{I}} \cdot \hat{\mathbf{J}})^{2}+\frac{3}{2} \hat{\mathbf{I}} \cdot \hat{\mathbf{J}}-I(I+1) J(J+1)}{2 J I(2 J-1)(2 I-1)}
$$

where $I$ is the quantum number of nuclear spin, $A_{\mathrm{HFS}}$ is the magnetic-dipole hyperfine constant, and $B_{\mathrm{HFS}}$ is the electric-quadrupole hyperfine constant. Their values are 
Table 7.1: Rb hyperfine structure constants for $5 \mathrm{P}_{3 / 2}$ [3, 4].

\begin{tabular}{l|l|l}
\hline & $A_{\mathrm{HFS}}$ & $B_{\mathrm{HFS}}$ \\
\hline $\mathrm{Rb}^{87}$ & $h \cdot 84.7185(20) \mathrm{MHz}$ & $h \cdot 12.4965(37) \mathrm{MHz}$ \\
$\mathrm{Rb}^{85}$ & $h \cdot 25.0020(99) \mathrm{MHz}$ & $h \cdot 25.790(93) \mathrm{MHz}$ \\
\hline \hline
\end{tabular}

listed in Table 7.1. The octupole is omitted from the calculation, since its contribution is so small that we would not be able to experimentally see the effect.

In the calculation of the matrix elements of the full Hamiltonian, we use,

$$
\begin{array}{r}
\hat{\mathbf{I}} \cdot \hat{\mathbf{J}}=I_{z} J_{z}+\frac{1}{2}\left(\hat{I}_{+} \hat{J}_{-}+\hat{I}_{-} \hat{J}_{+}\right) \\
\hat{J}_{ \pm}\left|J, J_{z}\right\rangle=\hbar \sqrt{J(J+1)-J_{z}\left(J_{z} \pm 1\right)}\left|J, J_{z} \pm 1\right\rangle \\
\hat{I}_{ \pm}\left|I, I_{z}\right\rangle=\hbar \sqrt{I(I+1)-I_{z}\left(I_{z} \pm 1\right)}\left|I, I_{z} \pm 1\right\rangle
\end{array}
$$

Although the $\left|I J m_{I} m_{J}\right\rangle$ basis is "bad" in the weak field regime, the computer program LAPACK (Linear Algebra PACKage), which diagonalizes matrices regardless of the basis, returns the eigenvalues of the full Hamiltonian. Fig. 7.1 shows the calculated light shift of the transitions $5 \mathrm{~S}_{1 / 2} \rightarrow 5 \mathrm{P}_{3 / 2}$ for the isotopes of rubidium, ${ }^{85} \mathrm{Rb}$ and ${ }^{87} \mathrm{Rb}$, as a function of lattice laser intensity, which is proportional to $E_{0}^{2}$.

Weak field regime. When the lattice intensity is low, $\hat{H}_{\mathrm{HFS}}$ dominates, and $\left|F, m_{F}\right\rangle(\hat{\mathbf{F}}=\hat{\mathbf{J}}+\hat{\mathbf{I}})$ is the "good" basis. The light shift of $5 \mathrm{~S}_{1 / 2}$ and $5 \mathrm{P}_{3 / 2}$ levels are,

$$
\begin{aligned}
\Delta 5 \mathrm{~S} & =-\frac{1}{4} \alpha_{5 \mathrm{~S}} E_{0}^{2} \\
\Delta 5 \mathrm{P} & =-\frac{1}{4} \alpha_{0} E_{0}^{2}-\frac{1}{4} \alpha_{2} E_{0}^{2} \frac{3 m_{F}^{2}-F(F+1)}{F(2 F-1)}
\end{aligned}
$$

All sub levels of the ground state $5 \mathrm{~S}_{1 / 2}$ has the same a.c. shift. The number of $5 \mathrm{P}_{3 / 2}$ states is,

- ${ }^{87} \mathrm{Rb}\left(I=\frac{3}{2}, J=\frac{3}{2}\right), F=0,1,2,3$, total number is $1+3+5+7=16$

- ${ }^{85} \mathrm{Rb}\left(I=\frac{5}{2}, J=\frac{3}{2}\right), F=1,2,3,4$, total number is $3+5+7+9=24$ 

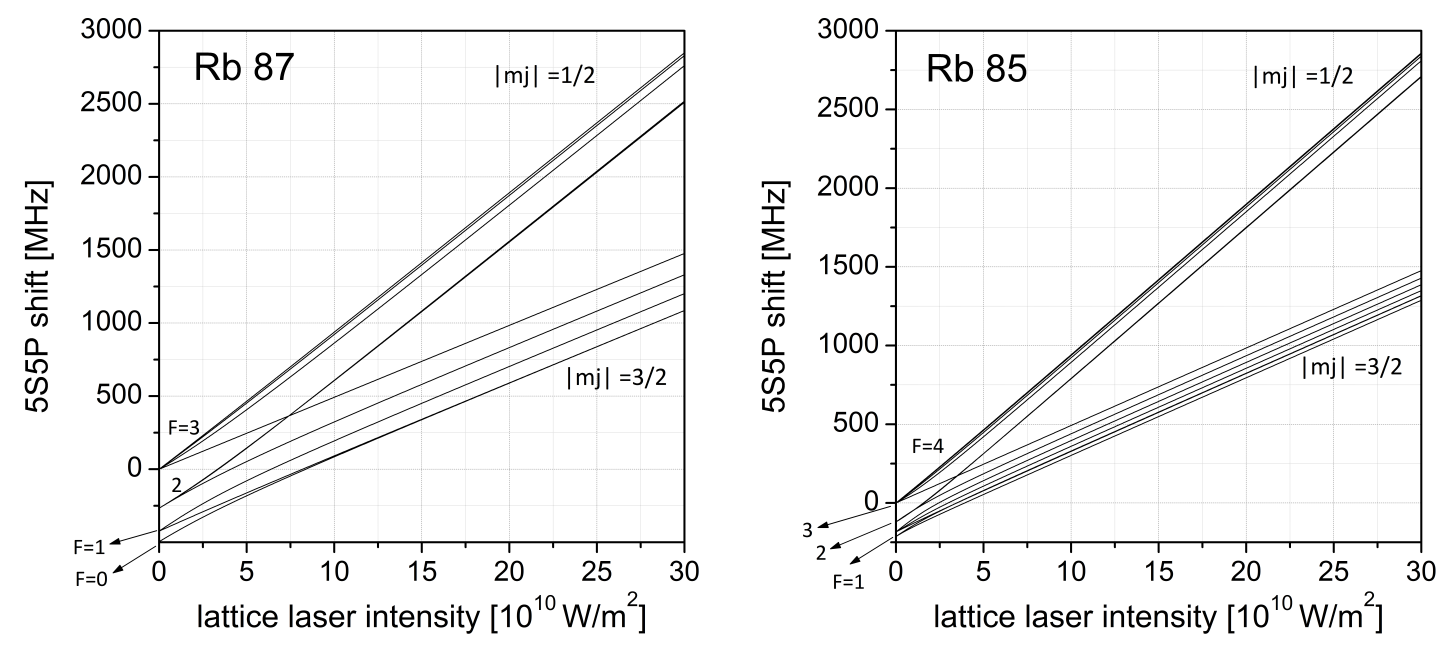

Figure 7.1:

The a.c. Stark light shift of the transitions $5 \mathrm{~S}_{1 / 2} \rightarrow 5 \mathrm{P}_{3 / 2}$ of ${ }^{87} \mathrm{Rb}$ $\left({ }^{85} \mathrm{Rb}\right)$ relative to the on-resonance cycling transition $5 \mathrm{~S}_{1 / 2}, \quad F=$ $2(3) \rightarrow 5 \mathrm{P}_{3 / 2}, \quad F^{\prime}=3(4)$. The plotted energy level shift is a combination of the light shifts of these two levels. The ground-state $5 \mathrm{~S}_{1 / 2}$ sublevels have the same a.c. shift, and the splitting here are caused by the upper level $5 \mathrm{P}_{3 / 2}$. The calculation uses $\alpha_{5 \mathrm{~S}}\left(5 \mathrm{~S}_{1 / 2}\right)=750 \times 4 \pi \epsilon_{0} \mathrm{a}_{0}^{3}$, $\alpha_{0}\left(5 \mathrm{P}_{3 / 2}\right)=-800 \times 4 \pi \epsilon_{0} \mathrm{a}_{0}^{3}$, and $\alpha_{2}\left(5 \mathrm{P}_{3 / 2}\right)=500 \times 4 \pi \epsilon_{0} \mathrm{a}_{0}^{3}$ as a trial number in the Hamiltonian. Although later-on with the experimental data input, we know these number are not accurate, but qualitatively the level splitting and crossing behavior is correct.

The levels with the same $F$ and $\left|m_{F}\right|$ value are degenerate. The light shift is proportional to $E_{0}^{2}$, so the energy levels look linearly when plotted as a function of lattice laser intensity. As can be seen in Fig. 7.1, the levels start from four groups of different $F$. Although the above equation is only "good" in the weak-field regime for all of the sublevels, this relation is still correct in the strong field regime for the states with the highest $\left(F,\left|m_{F}\right|\right)$ values. As shown in Fig. 7.1, the energy level of the states with highest $\left(F,\left|m_{F}\right|\right)$ is linear in the entire field strength range. This is because in Eq. 7.4 $\hat{\mathbf{I}} \cdot \hat{\mathbf{J}}$ does not couple to other levels.

Intermediate field strength. As the lattice power increases, neither $\hat{H}_{\mathrm{HFS}}$ or $\hat{H}_{\mathrm{E}}$ dominates. The energy levels generally turn curvy and a crossing occurs. The location where the cross happens depends on the value of the polarizabilities: $\alpha_{0}$ and 
$\alpha_{2}$ determine the significance of the field strength relative to the hyperfine structure in the full Hamiltonian.

"Paschen-Back", strong field regime. When the lattice intensity is high and $\hat{H}_{\mathrm{E}}$ dominates, $\left|J I m_{J} m_{I}\right\rangle$ becomes the "good" basis, and the light shift is described by Eq. 7.2. The ground state $5 \mathrm{~S}_{1 / 2}$ has no a.c.-split sublevels, while the number of $5 \mathrm{P}_{3 / 2}$ sublevels is, again,

- ${ }^{87} \mathrm{Rb}, J=\frac{3}{2}, I=\frac{3}{2}$, number of levels $=\left(2 \times \frac{3}{2}+1\right)\left(2 \times \frac{3}{2}+1\right)=4 \times 4=16$

- ${ }^{85} \mathrm{Rb}, J=\frac{3}{2}, I=\frac{5}{2}$, number of levels $=\left(2 \times \frac{3}{2}+1\right)\left(2 \times \frac{5}{2}+1\right)=4 \times 6=24$

The energy levels become linear functions of $E_{0}^{2}$ again on the plot, and they separate into two groups based on their $\left|m_{J}\right|$ value. Since $\alpha_{2}>0$, the group with higher energy includes levels with $\left|m_{J}\right|=\frac{1}{2}$, while the group with lower energy has $\left|m_{J}\right|=$ $\frac{3}{2}$. This is because the $J_{z}^{2}$ in $\hat{H}_{\mathrm{E}}$ determines the difference in energy between each subgroups. Inside each subgroup, the energy levels splitting is determined by the hyperfine perturbation. For the case of ${ }^{87} \mathrm{Rb}, I=\frac{3}{2}$ and $J=\frac{3}{2}$

- $\left|m_{J}\right|=\frac{3}{2} \cdot\left\langle J J_{z}\left|\hat{I_{+}} \hat{J_{-}}+\hat{I_{-}} \hat{J_{+}}\right| J J_{z}\right\rangle=0$, all the off-diagonal terms vanishes in $\hat{\mathbf{I}} \cdot \hat{\mathbf{J}}$ and $(\hat{\mathbf{I}} \cdot \hat{\mathbf{J}})^{2}$, and the energy levels splitting is determined by the diagonal terms, which are proportional to $I_{z} J_{z}= \pm \frac{9}{4}$ or $\pm \frac{3}{4}$. The levels appear nearly equally spaced on the plot, due to the contribution from $A_{\mathrm{HFS}} \hat{\mathbf{I}} \cdot \hat{\mathbf{J}}$, the magnetic dipole. The small deviation from an exact equal spacing is due to the perturbation of electric quadruple term, whose strength is a few percent of that of the magnetic dipole term.

- $\left|m_{J}\right|=\frac{1}{2} .\left\langle J J_{z}\left|\hat{I_{+}} \hat{J_{-}}+\hat{I_{-}} \hat{J}_{+}\right| J J_{z}\right\rangle$ are not all vanishing. $\hat{\mathbf{I}} \cdot \hat{\mathbf{J}}$ has off-diagonal terms. The energy-levels splittings are not as straightforward as in the case of $\left|m_{J}\right|=\frac{3}{2}$, but can be numerically computed, of course.

The values of $\alpha_{0}$ and $\alpha_{2}$ are the same for ${ }^{85} \mathrm{Rb}$ and ${ }^{87} \mathrm{Rb}$. For the case of ${ }^{85} \mathrm{Rb}$, $I=\frac{5}{2}$ and $J=\frac{3}{2}$. The energy levels also separate into two subgroups. The group is 
higher in energy has $\left|m_{J}\right|=\frac{1}{2}$, and the one lower in energy has $\left|m_{J}\right|=\frac{3}{2}$. Similar to the case of ${ }^{87} \mathrm{Rb}$, the group of $\left|m_{J}\right|=\frac{3}{2}$ appears nearly equally spaced on the plot. $\left(I_{z} J_{z}= \pm \frac{15}{4}, \pm \frac{9}{4}\right.$, or $\pm \frac{3}{4}$.)

The theoretical calculation of a.c. Stark shift for the rubidium cycling transition is not complicated once the dynamic polarizability values of $5 \mathrm{~S}_{1 / 2}$ and $5 \mathrm{P}_{3 / 2}$ levels are given. The real difficulty is in the theoretical calculation of the dynamic polarizability. The theoretical calculation of the rubidium in $5 \mathrm{~S}_{1 / 2}$ dynamic polarizability has less uncertainty, a recently calculated value is $687.3(5) \times 4 \pi \epsilon_{0} a_{0}^{3}$ [40, 46, 47]; however, the available experimental measurement concludes a value at $769 \pm 62 \times 4 \pi \epsilon_{0} a_{0}^{3}[48$. The calculation of the rubidium $5 \mathrm{P}_{3 / 2}$ dynamic polarizability is even more complicated - in addition to the contribution of the $p-s$ transitions, the theorists also have to include the $p-d$ transition matrix elements in their calculation. The experimental determination of the dynamic polarizability of both of $5 \mathrm{~S}_{1 / 2}$ and $5 \mathrm{P}_{3 / 2}$ levels is not straight-forward, either, mostly due to the challenges in the calibration of electric field experienced by the cold atoms at the trapping region. For the measurement of the $5 \mathrm{P}_{3 / 2}$ polarizability, an additional challenge is that the field strength in most of the experiments is in the weak-field regime, where the levels of the $5 \mathrm{P}_{3 / 2}$ are not easily resolvable. So far the values of the rubidium $5 \mathrm{P}_{3 / 2}$ dynamic polarizabilities $\alpha_{0}$ and $\alpha_{2}$ at $1064 \mathrm{~nm}$ are not well-determined, despite the fact that the $1064 \mathrm{~nm}$ dipole trap involving rubidium $5 \mathrm{~S}_{1 / 2} \rightarrow 5 \mathrm{P}_{3 / 2}$ transition is widely used.

I note that there also exists an $\alpha_{1}$, vector polarizability, but it only leads to measurable effects in field with a circular component, which is not the case in my experiment.

In my experiment, the lattice intensity is very high such that the lattice-induced light shift is in the Paschen-Back regime. With such an outstanding light shift, the sublevels are easily resolvable, and they are also linearly related to $E_{0}^{2}$. In the following section, we use the spectral lines to obtain experimental values of $\alpha_{0}$ and $\alpha_{2}$. The beauty of the spectroscopy method presented here is that the determination of $\alpha_{0}$ 
(a)

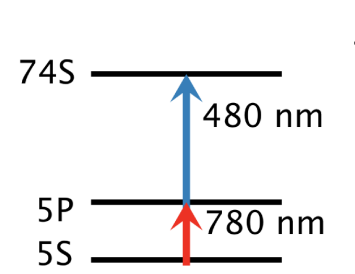

(b)

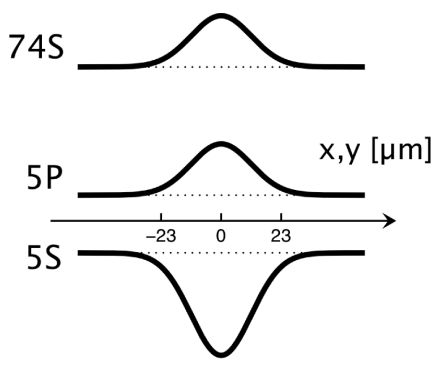

(c)

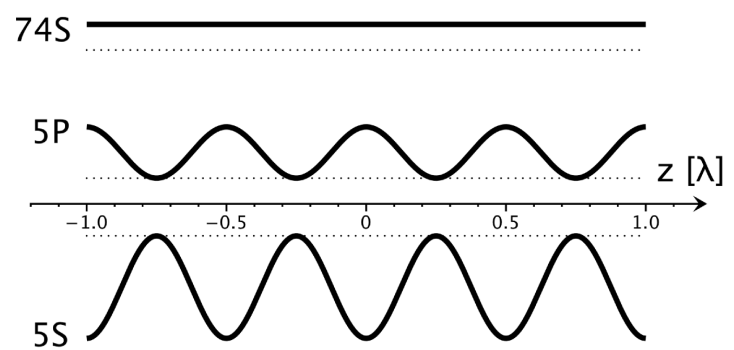

Figure 7.2:

Relevant levels of a two-photon Rydberg excitation. Dotted lines are unshifted energy levels, while the solid lines are lattice-shifted levels. The gaps between levels are not to scale. (a) No light-shift, on-resonance twophoton excitation. (b)(c) With light shift in a $1064 \mathrm{~nm}$ lattice. The $74 \mathrm{~S}$ state has a constant light shift along z-axis.

and $\alpha_{2}$ turns out to be insensitive to the calibration of lattice intensity.

\subsection{Two-photon excitation in deep optical lattices: numeri- cal simulation}

The following discussion is specific for the experimental measurement of polariz-

abilities of a ${ }^{87} \mathrm{Rb}$ atom sample, but the general rules also apply to the case of ${ }^{85} \mathrm{Rb}$; we choose to use ${ }^{87} \mathrm{Rb}$, because the number of $m_{j}$ sublevels is less, and the residual hyperfine splitting in the Paschen-Back regime is larger than that of ${ }^{85} \mathrm{Rb}$.

\subsection{1 "Magic" Rydberg state}

In a $1064 \mathrm{~nm}$ lattice, both $5 \mathrm{~S}_{1 / 2}$ and $5 \mathrm{P}_{3 / 2}$ have local response to the lattice light field, i.e. their light shifts are given by,

$$
-\frac{1}{4} \alpha\left|E_{0}(\mathbf{R})\right|^{2}
$$


where $E_{0}(\boldsymbol{R})$ is the lattice field amplitude at the center-of-mass location of the atom. Rydberg atoms have a non-local response to the field, and the light shift is given by,

$$
V_{\text {ad }}(\mathbf{R})=-\frac{1}{4} \alpha_{e} \int\left|E_{0}(\mathbf{R}+\mathbf{r})\right|^{2}|\psi(\mathbf{r})|^{2} d^{3} r
$$

where $\alpha_{e}=-545 \times 4 \pi \epsilon_{0} a_{0}^{3}$, calculated from the free-electron ponderomotive energy. This means that the free-electron response is averaged over the atom, using $|\psi(\mathbf{r})|^{2}$ as a weighting factor. The averaging in z-direction is important, because in this direction the size of a Rydberg atom approximately equals the lattice period. The averaging in $\rho$ direction is not important, because the cavity mode waist $w_{0}$ is much greater than the size of a Rydberg atom. For a few state, $V_{\mathrm{ad}}$ only depends on $\rho$ and not on $z$. For rubidium in $1064 \mathrm{~nm}$ lattice, one such "magic" state is $74 \mathrm{~S}_{1 / 2}$ state. For the magic states,

$$
V_{\mathrm{ad}}(\rho)=-\frac{1}{4} \frac{\alpha_{e}}{2} E_{0, M}^{2}(\rho)=-\frac{1}{4} \times(-272.5) \times E_{0, M}^{2}(\rho)
$$

Where $E_{0, M}^{2}$ is the field amplitude at a distance $\rho$ from the lattice axis.

We utilize two-photon excitation spectroscopy of the magic Rydberg state in $1064 \mathrm{~nm}$ lattices to measure the dynamic polarizability. At low filed, the lower transition is $5 \mathrm{~S}_{1 / 2}(\mathrm{~F}=2) \rightarrow 5 \mathrm{P}_{3 / 2}\left(F^{\prime}=3\right)(780 \mathrm{~nm}$, red), and the upper transition is $5 \mathrm{P}_{3 / 2}\left(F^{\prime}=3\right) \rightarrow 74 \mathrm{~S}$ magic state $(480 \mathrm{~nm}$, blue). As shown in see Fig. 7.2 , the constant light shift of the 74S Rydberg magic state along z-axis simplifies the spectroscopic determination of the dynamic polarizabilities and the numerical simulation for model-fitting the data.

The spectra are taken under a fixed lattice power and a fixed $5 \mathrm{~S}_{1 / 2} \rightarrow 5 \mathrm{P}_{3 / 2}$ detuning $(\Delta \mathrm{red})$. The detected lattice-shifted signal is recorded as a function of $5 \mathrm{P}_{3 / 2} \rightarrow 74 \mathrm{~S}$ transition detuning compared to the off-resonance unshifted MOT atom signal ( $\Delta$ blue). Fig. $7.3(\mathrm{~b})$ shows an illustration of the relevant detunings.

The energy levels in Fig. 7.1 do not immediately match the experimental data. 
To do the model fitting and extract the values of dynamic polarizability from the experimental data, we simulate the spectral lines based on the energy level calculation. The simulation has two major steps: first, seeking the two-photon excitation resonances, and second, accounting for the center-of-mass thermal motion of the atoms in the optical lattice which may cause the spectral line broadening. Doppler shifts are negligible and are not included in the model.

\subsubsection{Two-photon excitation resonances}

The diagonalization of the full Hamiltonian $\hat{H}=\hat{H}_{\mathrm{HFS}}+\hat{H}_{\mathrm{E}}$ gives energy level plots as in Fig. 7.1. Inside the deep optical lattice trap, the atoms see a dramatic spatial variation of lattice laser intensity. The spatial variation includes the standing wave patterns along the lattice axis, and the exponential decrease of intensity due to the waist $(23 \mu \mathrm{m})$ of the laser beam (Fig. 4.1 shows the geometry). As a result, in a single spectroscopic scan, there are usually several on-resonant excitation signals from atoms located at different regions of different lattice intensities. The first step of reconstructing the experimental data is thus finding all the on-resonant excitation spots inside the deep optical lattice.

In the energy level plot, for each $5 \mathrm{~S}_{1 / 2} \rightarrow 5 \mathrm{P}_{3 / 2}$ detuning ( $\Delta$ red), we find the lattice intensities at which the $5 \mathrm{~S}_{1 / 2} \rightarrow 5 \mathrm{P}_{3 / 2}$ transition is on-resonance to one of the light-shifted $5 \mathrm{P}_{3 / 2}$ sublevels. The $5 \mathrm{~S}_{1 / 2}$ sub levels all have the same light shift. Red circles in Fig. 7.3(a) mark such resonances. From each lattice intensity marked, we calculate the upper transition detuning ( $\Delta$ blue) that is on resonance with latticeshifted $5 \mathrm{P}_{3 / 2} \rightarrow 74 \mathrm{~S}$ transition. $\Delta$ blue is defined as the frequency shift of the $480 \mathrm{~nm}$ in a spectrum, measured from the center of the narrow, off-resonance signal of MOT atoms, to the center of the broadened, on-resonance signal of atoms in the lattice. 
(a)

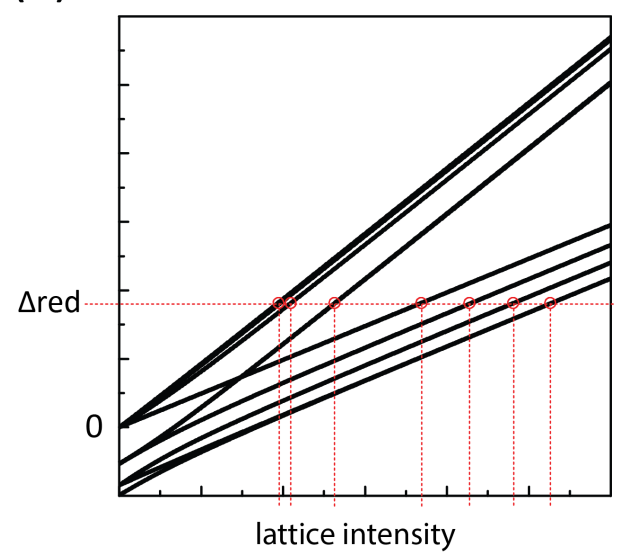

(b)

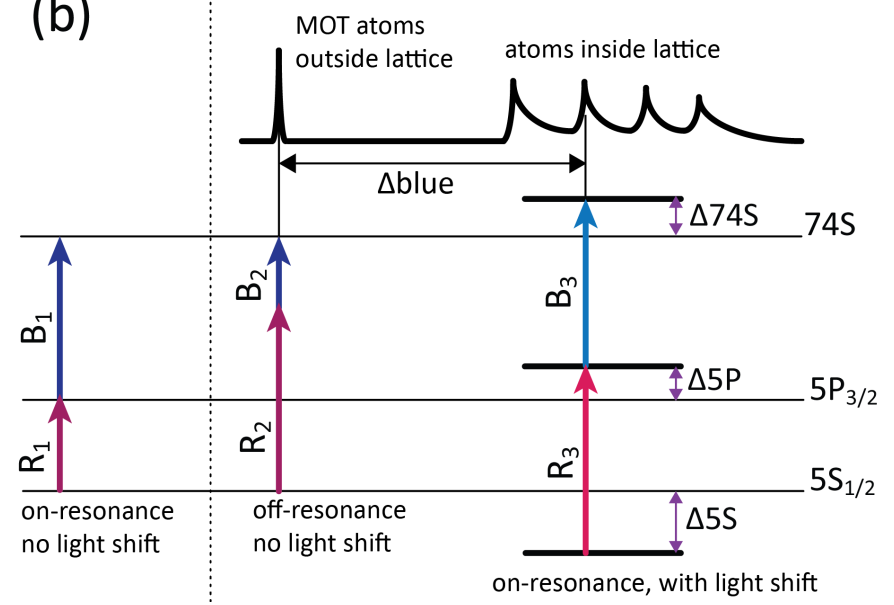

Figure 7.3:

Illustration of the resonance-seeking. (a) The open circles mark the resonances. When the lower-transition detuning relative to the cycling transition is $\Delta$ red, the energy levels are on resonance with the lower transition at different lattice intensities. (b) Top: sketch of a two-photon excitation spectrum with fixed lower transition detuning $\Delta$ red. Bottom: energy levels. Transition frequencies: $R_{1}$ is the on-resonance cycling transition $\left(5 \mathrm{~S}_{1 / 2} \rightarrow 5 \mathrm{P}_{3 / 2}\right)$, and $\mathrm{B}_{1}$ is the on-resonance upper transition frequency. $\mathrm{R}_{2}=\mathrm{R}_{3}=\mathrm{R}_{1}+\Delta$ red $=\mathrm{R}_{1}+\Delta 5 \mathrm{~S}+\Delta 5 \mathrm{P} . \Delta$ blue $=\mathrm{B}_{3}-\mathrm{B}_{2}=\Delta 74 \mathrm{~S}+\Delta 5 \mathrm{~S}$

See Fig. $7.3(\mathrm{~b})$ for an illustration. We calculate $\Delta$ blue by,

$$
\begin{aligned}
\Delta \text { blue } & =\mathrm{B}_{3}-\mathrm{B}_{2} \\
& =\Delta 74 \mathrm{~S}+\Delta 5 \mathrm{~S} \\
& =\Delta 74 \mathrm{~S}+\Delta \text { red }-\Delta 5 \mathrm{P}
\end{aligned}
$$

where $\Delta 74 \mathrm{~S}=-\frac{1}{4} \alpha_{74 \mathrm{~S}} E_{0}^{2}$ and $\alpha_{74 \mathrm{~S}}=-\frac{1}{2} 545 \times 4 \pi \epsilon_{0} a_{0}^{3}$, calculated from the freeelectron ponderomotive shift [19], averaged over one lattice period along the lattice axis.

Repeating the same resonance-seeking procedure at different values of $\Delta$ red, we find all the resonance spots in a lattice with an on-axis intensity maximum $I_{0}$. Fig. 7.4 shows a plot of all the resonances. The y-axis shows $\Delta$ red in $\mathrm{MHz}$, and the $\mathrm{x}$-axis shows the $\Delta$ blue in $\mathrm{MHz}$. The resonances for each sublevels disappear at the same 


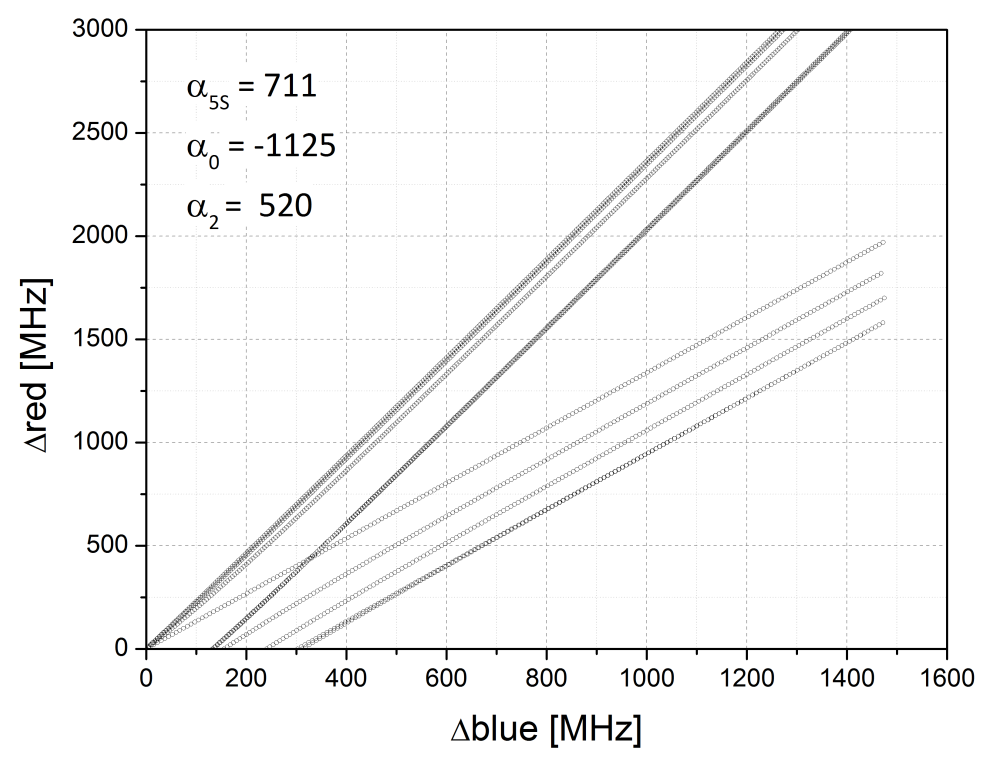

Figure 7.4:

Calculated two-photon excitation spectra for the $74 \mathrm{~S}$ magic state of ${ }^{87} \mathrm{Rb}$ in a $1064 \mathrm{~nm}$ lattice. The plot shows discrete resonances spots.

maximum $\Delta$ blue $\approx 1440 \mathrm{MHz}$. This is because the upper limit is set by $\Delta 74 \mathrm{~S}+\Delta 5 \mathrm{~S}$, regardless of the $\Delta$ red of the different sublevels. The resonances separate into two groups. The group with larger $\Delta$ red has $\left|m_{j}\right|=\frac{1}{2}$, while the one lower in $\Delta$ red has $\left|m_{j}\right|=\frac{3}{2}$. The slopes of these two groups provide the information on $\alpha_{0}$ and $\alpha_{2}$. See Sec. 7.3 .3 for details, which we exploit to experimentally determine their values.

\subsubsection{Spectral line broadening}

The calculation in Sec. 7.2 .2 shows the spectral features, qualitatively. However, this calculation only finds the resonance points and neglects all the possible line broadening effects. To give a better model of the experimental data, we have to include both the off-resonance excitation and line broadening from various sources. In the end result, we plot simulated $\Delta$ blue- $\Delta$ red spectra as a $301 \times 301$ pixelated picture. The value of each pixel represents the count rate as a function of both detunings ( $\Delta$ blue, $\Delta$ red). Fig. 7.5 shows a typical plot of such pixelated picture.

In the simulation, the lattice is divided into concentric shells about the lattice 
axis. The lattice intensity of each shell at a radial distance $\rho$ from the lattice axis is $I(\rho)=I_{0} \exp \left[\frac{-2 \rho^{2}}{w_{0}^{2}}\right]$. The count rate of each pixel in the picture is first calculated inside an intensity shell. The calculation is repeated radially outward for each shell, and the count rates from different shells are normalized with a weighting factor which is proportional to the volume of the shell and the lower-transition intensity in the shell. (The lower transition $780 \mathrm{~nm}$ laser co-propagates with the lattice laser in the experiment.) The pixel values on the picture are accumulated as the shell radius is varied.

Inside each lattice intensity shell, the computer program scans the energy level plot in Fig. 7.1 with $\Delta$ red steps corresponding to the pixel size in the final picture. At each $\Delta$ red step, the corresponding values of $\Delta$ blue are calculated for lattice intensites ranging from here to $I(\rho)$. This full range of lattice intensity coverage is due to the fact that, inside each shell, the lattice intensity has periodic standing wave patterns

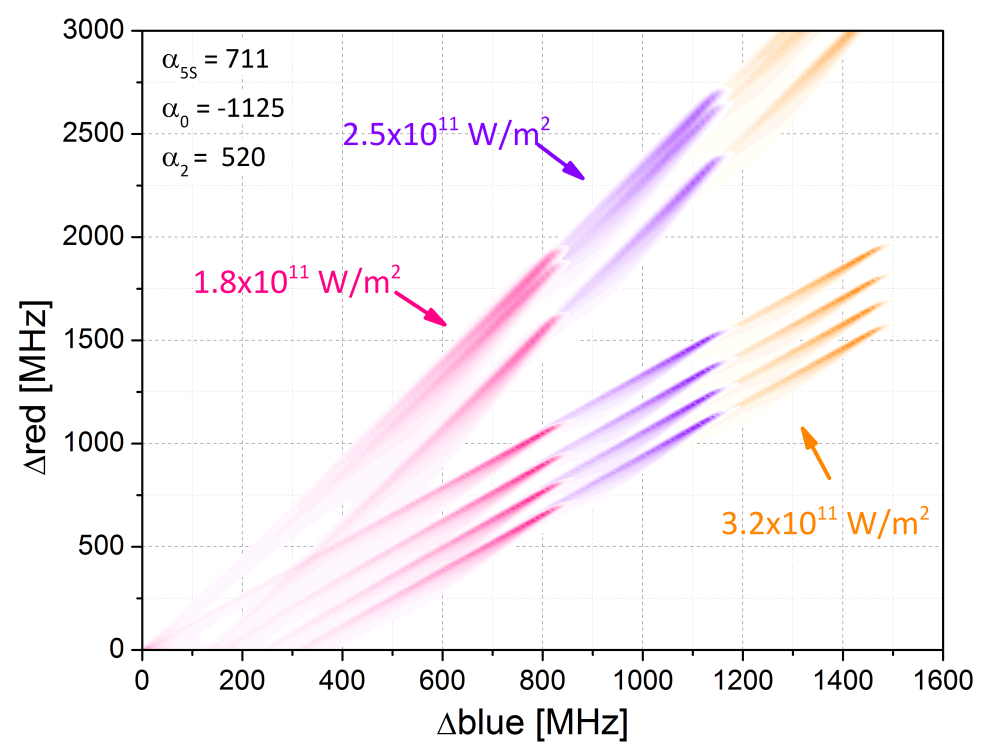

Figure 7.5:

Calculated two-photon excitation spectra for 74S magic state in $1064 \mathrm{~nm}$ lattice. The plot includes the effects of off-resonant excitation. The slope of the spectral lines are insensitive to the lattice power, as seen in the plot. Note that we have overlaid the results obtained for three different peak lattice intensities in the same plot). 
along the lattice axis. The intensity varies sinusoidally as a function of $\mathrm{z}$, from zero to the maximum $I(\rho)$. As the program steps through all $\Delta$ blue values for the entire range of lattice intensity instead of just looking for discrete resonant spots, both onresonantly and off-resonantly excited 5P intermediate-level populations contribute to the final $74 \mathrm{~S}$ signal. The count rate for $\Delta$ red and a intensity $I=I(\rho, z)$ is, signal $=$ Boltzmann factor $\times$ saturation parameter $\times$ z-weighting factor

In the following I explain all factors.

The Boltzmann factor is calculated as,

$$
\exp \left[\frac{-\frac{1}{4} E_{0}^{2} \alpha_{5 \mathrm{~S}}+\frac{1}{4} E(\rho, z)^{2} \alpha_{5 \mathrm{~S}}}{k_{\mathrm{B}} T}\right]
$$

$E_{0}=\sqrt{\frac{2 I_{0}}{c \epsilon_{0}}}$ is the maximum of the field amplitude inside the entire lattice trap. $E(\rho, z)$ is the field amplitude at the atom's location $(\rho, z)$. The Boltzmann factor accounts for the line broadening due to the thermal atom number density distribution inside the $5 \mathrm{~S}$ lattice potential wells. For the temperature, $T$, we typically assume several milli-Kelvin.

The saturation parameter is calculated by,

$$
\mathrm{S}=\frac{1}{2} \frac{\mathrm{s}}{1+\mathrm{s}+4\left(\frac{\Delta}{6 \mathrm{MHz}}\right)^{2}}
$$

with $\Delta=$ (on-resonance lattice-shifted transition frequency of $\left.5 \mathrm{~S}_{1 / 2} \rightarrow 5 \mathrm{P}_{3 / 2}\right)-\Delta$ red. $\mathrm{s}=\frac{I_{r e d}}{I_{s a t}} \exp \left[\frac{-2 \rho^{2}}{w_{r}^{2}}\right] . \quad w_{r}^{2}$ is the beam waist of the lower transition laser, which copropagates with the lattice laser. The value of $\mathrm{S}$ properly weights the count rate contribution from on-resonance and off-resonance excitations. For the case of onresonant excitation, $\Delta=0$, the lower transition frequency matches the lattice-shifted $5 \mathrm{~S}_{1 / 2} \rightarrow 5 \mathrm{P}_{3 / 2}$ transition, and the signal contribution is the largest. For the case of off-resonant excitation, there is still population excited to 5P state due to saturation broadening. In both cases, the upper transition laser detunig is calculated as the 
frequency detuning needed to bring atoms from the lattice-shifted intermediate $5 \mathrm{P}_{3 / 2}$ state to the Rydberg state.

The z-weighting parameter is proportional to the "volume" inside the shell with

the lattice intensity $I(\rho, z)$. The intensity is mapped into z-coordinate by an inverse cosine relation in the calculation of the volume. This z-weighting factor accounts for the sinusoidal intensity variation along z-axis inside the cylindrical shell.

Although the model is relatively simple, the final $\Delta$ red- $\Delta$ blue picture strongly agrees with the spectral features in the experimental data, as presented in Fig. 7.6. The calculation also shows that the spectral features are insensitive to the lattice power: the spectral lines on the $\Delta$ red- $\Delta$ blue plot separate into two groups, and the slopes of the spectral lines and the location of the crossing on the plot is independent of the lattice power. The lattice power and the peak lattice intensity do make a difference in the spectral signal strength distribution on the $\Delta$ red- $\Delta$ blue plot. With lower lattice intensity, the signal at lower $\Delta \operatorname{red}-\Delta$ blue is stronger, whereas the signal strength for a higher lattice intensity tends to concentrate in the region with larger $\Delta$ blue. This behavior is due to the Boltzmann factor. There is always a sharp cutoff at a certain $\Delta$ blue where the spectral lines end. The location of this cutoff depends on the peak lattice laser intensity. In principle, by matching the location of the cutoff and where the actual experimental spectral lines ends, we can calibrate the lattice intensity in the atom trapping region.

\subsection{Polarizability measurement}

\subsubsection{Methods}

We take several two-photon excitation spectra at a fixed lattice power with different $\Delta$ red. The count rate of each spectroscopic scan is recorded as a function of $\Delta$ blue, and then the spectra are plotted together in a waterfall plot. On the water- 


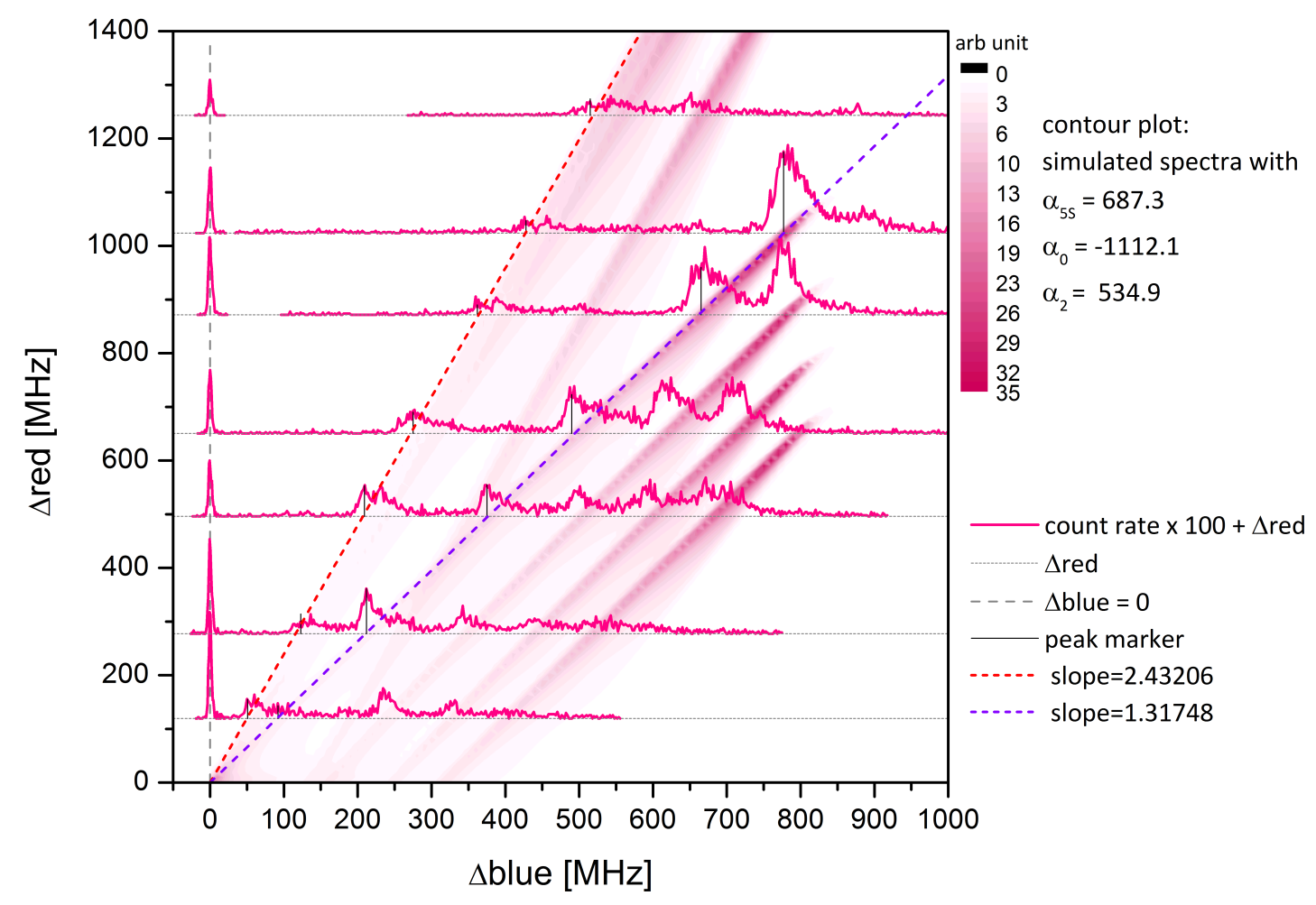

Figure 7.6:

Experimental spectra overlaid over a simulated contour plot. Experiment: lattice power $20 \mathrm{~mW}$, excitation pulse duration $2 \mu \mathrm{s}$. Simulation: lattice intensity $1.8 \times 10^{11} \mathrm{~W} / \mathrm{cm}^{2}$

fall plot, the spectra at different $\Delta$ red are offset by plotting count rate $\times 100+\Delta$ red. (The factor 100 simply is a convenient scaling factor). Fig. 7.6 shows the scans at $I_{0}=20 \mathrm{~mW}$. The uncertainty of the $\Delta$ red is less than $2 \%$, and $\Delta$ blue is less than $2 \%$. The uncertainty of lattice power calibration is about $8 \%$; however, the lattice power does not significantly affect the determination of the $\alpha_{0}$ and $\alpha_{2}$, because the slope of the spectral features are independent of the maximum lattice intensity.

The data taking rate of the two-photon excitation spectroscopy is $100 \mathrm{~Hz}$. Each point of the spectra is an average of 50 shots. During the first $9.5 \mathrm{~ms}$ in an experimental cycle, the lattice power is kept as low as $2 \mathrm{~mW}$ (loading power) to maximize the loading of the lattice with MOT atoms. The MOT laser is switched off at some point 
in the experimental cycle, and then the lattice starts compressing the atom sample. The compression is achieved by increasing the lattice power in $30 \mu$ s to $20 \mathrm{~mW}$. The increase of the lattice power follows a smooth sine-rising curve. The lattice power is held constantly high while the Rydberg excitation pulses are on. After the excitation, the lattice power is switched back to the loading power. The 74S Rydberg atoms get field-ionized and the positive ions are detected by a MCP detector. Details of the lattice compression can be found in the previous Chapter $\mathrm{V}$.

\subsubsection{Spectral line shape}

In this experiment, the two-photon excitation spectral-line shapes do not follow a common Lorentzian or Gaussian line shape, because the magnitude of the latticeinduced light shift dramatically varies in the atom trapping region. The following is a qualitatively explanation: along the lattice axis, the z-axis, the lattice laser intensity sinusoidally varies with a periodicity of $\frac{\lambda}{2}=532 \mathrm{~nm}$. At the position of nodes, i.e. $z= \pm \frac{\lambda}{2}, \pm \frac{3 \lambda}{2}, \cdots$, the lattice intensity cancels inside the cavity. In the transverse direction, i.e., the $\mathrm{x}$ - and $\mathrm{y}$-axes, the lattice intensity follows a Gaussian

profile $I(x)=I_{0} \exp \left(\frac{-2 x^{2}}{w_{0}^{2}}\right)$ with a beam waist of $23 \mu \mathrm{m}$. On any plane that is contains the lattice axis, the lattice intensity distribution can be described by "equal intensity ellipses", as shown in Fig. 7.7(a). At the origin, the lattice laser intensity is the maximal intensity, $I_{0}$. If we draw an equal-intensity line for intensity $I_{1}, I_{1}<I_{0}$, at a distance away from the origin, the shape of the loop mimics an ellipse because of the different dependence of $I$ on $\rho=\sqrt{x^{2}+y^{2}}$ and $\mathrm{z}$. the asymmetric between the z-axis and transverse direction. If we draw a line for another, lower intensity, $I_{2}, I_{2}<I_{1}$, which is further away from the origin, the "eccentricity" of the ellipse will increase, since in the radial direction the lattice power decreases slower than in the z-direction. The number of atoms that see a specific intensity $I$ is proportional to the volume of the elliptical shell which is at that intensity, and the corresponding Boltzmann factor. 
(a)

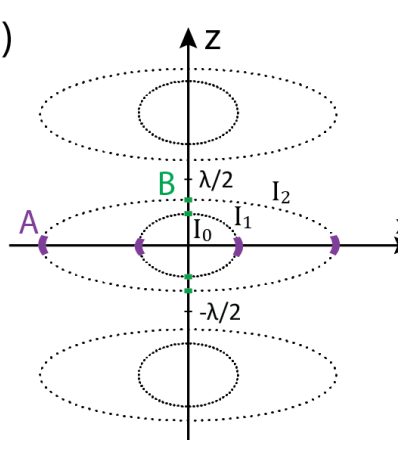

(b)

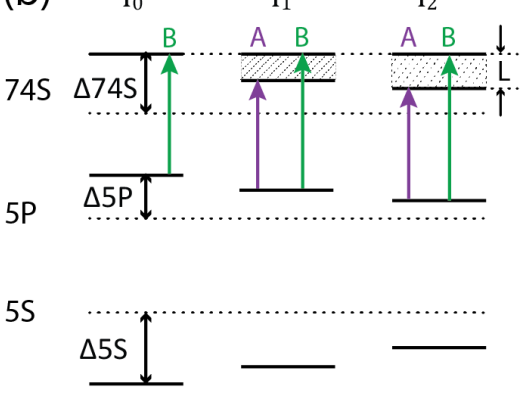

(c)

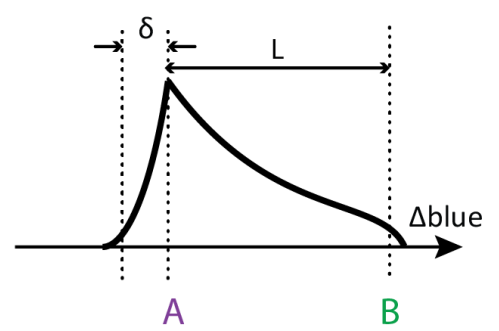

Figure 7.7:

(a) "Ellipses" of equal lattice laser intensity. Intensity: $I_{1}>I_{2}$, eccentricity: $\epsilon_{2}>\epsilon_{1}$. (b) Light shift of the magic Rydberg state. (c) triangular line shape: $\delta$ is due to the laser linewidth, and $\mathrm{L}$ is due to the lattice intensity variation in space.

The atoms in the same elliptical shell see the same lattice intensity, so they all have the same lower transition detunings and the same Boltzmann factor. However, the atoms on the same ellipse experience different lattice induced light shift for the 74S Rydberg magic state. The $74 \mathrm{~S}$ magic state has a constant light shift along the z-axis without any z-dependent modulation, and the magnitude of that constant light shift only depends on the amplitude of the lattice intensity, $I(\rho, z=0)=I_{0} \exp \left(\frac{-2 \rho^{2}}{\omega_{0}^{2}}\right)$ . The atoms with the same distance from the z-axis always see the same light shift of the $74 \mathrm{~S}$ state. As a result, on the same ellipse, there are two extreme cases: atoms at the major axis, point $\mathrm{A}$, see the minimal light shift of the $74 \mathrm{~S}$, while the atoms at the minor axis, point B, see the maximal light shift of the $74 \mathrm{~S}$ (see Fig. 7.7(a)). Also, at point A, the signal strength is at a maximum because the volume of the corresponding region is the largest, while at point B the signal strength is a minimum. The variation of the signal strength versus the $74 \mathrm{~S}$ light shift causes the triangular spectral line shape with a tail on the high frequency side.

As shown in Fig. 7.7 (b), the upper transition detunings on a ellipse varies by a frequency range of $\mathrm{L}$. The maximal $\mathrm{L}$ is determined by the lower transition detuning, because the $\Delta 5 \mathrm{~S}+\Delta 5 \mathrm{P}$ light shift has to approximately equal $\Delta$ red so that one of the light-shifted intermediate $5 \mathrm{P}_{3 / 2}$ levels is populated. The resonance occurs at 
a certain intensity $I_{\text {res }}$. The 74S light shift along the equal-intensity "ellipse" for $I_{\text {res }}$ then varies between $-\frac{1}{4} \alpha_{74 \mathrm{~S}} \frac{2}{c \epsilon_{0}} I_{0}$ at point $\mathrm{B}$ and $-\frac{1}{4} \alpha_{74 \mathrm{~S}} \frac{2}{c \epsilon_{0}} I_{\text {res }}$ at point $\mathrm{A}$ (see Fig. 7.7). The frequency range $\mathrm{L}$ therefore is,

$$
\mathrm{L}=-\frac{1}{4} \alpha_{74 \mathrm{~S}} \frac{2}{\mathrm{c} \epsilon_{0}}\left[\mathrm{I}_{0}-\mathrm{I}_{\mathrm{res}}\right]
$$

This finding accords with the observation that with smaller lower transition detunings $\Delta$ red the lattice spectral lines become wider, more asymmetric and triangular. For each lattice spectral line, we use the position of the maximal value as the primary marker for the spectral line. According to Fig. 7.7 and the explanation provided, the markers correspond to the A-locations on equal-intensity "ellipse".

\subsubsection{Experimental values of $\alpha_{0}$ and $\alpha_{2}$}

On the waterfall plot of the experimental spectra, the $\mathrm{x}$ and $\mathrm{y}$ axis are plotted as:

$$
\begin{aligned}
y & =\Delta \text { red } \\
& =\Delta 5 \mathrm{~S} 5 \mathrm{P} \\
& =\frac{1}{4}\left(\alpha_{5 \mathrm{~S}}-\alpha_{5 \mathrm{P}}\right) E_{0}^{2} \\
x & =\Delta \text { blue } \\
& =\Delta 74 \mathrm{~S}+\Delta 5 \mathrm{~S} 5 \mathrm{P}-\Delta 5 \mathrm{P} \\
& =\Delta 5 \mathrm{~S} 5 \mathrm{P}+\frac{1}{4}\left(\alpha_{5 \mathrm{P}}-\alpha_{74 \mathrm{~S}}\right) E_{0}^{2} \\
& =y\left(1+\frac{\alpha_{5 \mathrm{P}}-\alpha_{74 \mathrm{~S}}}{\alpha_{5 \mathrm{~S}}-\alpha_{5 \mathrm{P}}}\right) \\
& =y \frac{\alpha_{5 \mathrm{~S}}-\alpha_{74 \mathrm{~S}}}{\alpha_{5 \mathrm{~S}}-\alpha_{5 \mathrm{P}}}
\end{aligned}
$$


So the slope of the levels are,

$$
\frac{\mathrm{d} y}{\mathrm{~d} x}=\frac{\alpha_{5 \mathrm{~S}}-\alpha_{5 \mathrm{P}}}{\alpha_{5 \mathrm{~S}}-\alpha_{74 \mathrm{~S}}}
$$

There are two spectral lines that are linear in all field regimes and that are passing through the origin, as represented by the dashed lines in Fig. 7.6. They are the highest energy levels in each subgroup of $\left|m_{j}\right|=\frac{1}{2}$ and $\left|m_{j}\right|=\frac{3}{2}$, and their dynamic polarizabilities are calculated by:

$$
\begin{aligned}
& \alpha_{5 \mathrm{P}}\left(\left|m_{j}\right|=\frac{1}{2}\right)=\alpha_{0}+\alpha_{2} \frac{3 \times\left(\frac{1}{2}\right)^{2}-\frac{3}{2}\left(\frac{3}{2}+1\right)}{\frac{3}{2}\left(2 \times \frac{3}{2}-1\right)}=\alpha_{0}-\alpha_{2} \\
& \alpha_{5 \mathrm{P}}\left(\left|m_{j}\right|=\frac{3}{2}\right)=\alpha_{0}+\alpha_{2} \frac{3 \times\left(\frac{3}{2}\right)^{2}-\frac{3}{2}\left(\frac{3}{2}+1\right)}{\frac{3}{2}\left(2 \times \frac{3}{2}-1\right)}=\alpha_{0}+\alpha_{2}
\end{aligned}
$$

So their slopes are:

$$
\begin{aligned}
& \left.\frac{\mathrm{d} y}{\mathrm{~d} x}\right|_{\left|m_{j}\right|=\frac{1}{2}}=\frac{\alpha_{5 \mathrm{~S}}-\left(\alpha_{0}-\alpha_{2}\right)}{\alpha_{5 \mathrm{~S}}-\alpha_{74 \mathrm{~S}}} \\
& \left.\frac{\mathrm{d} y}{\mathrm{~d} x}\right|_{\left|m_{j}\right|=\frac{3}{2}}=\frac{\alpha_{5 \mathrm{~S}}-\left(\alpha_{0}+\alpha_{2}\right)}{\alpha_{5 \mathrm{~S}}-\alpha_{74 \mathrm{~S}}}
\end{aligned}
$$

From the differential slope and average slope, we get,

$$
\begin{aligned}
\left.\frac{\mathrm{d} y}{\mathrm{~d} x}\right|_{\left|m_{j}\right|=\frac{1}{2}}-\left.\frac{\mathrm{d} y}{\mathrm{~d} x}\right|_{\left|m_{j}\right|=\frac{3}{2}} & =\frac{2 \alpha_{2}}{\alpha_{5 \mathrm{~S}}-\alpha_{74 \mathrm{~S}}} \\
\frac{1}{2}\left(\left.\frac{\mathrm{d} y}{\mathrm{~d} x}\right|_{\left|m_{j}\right|=\frac{1}{2}}+\left.\frac{\mathrm{d} y}{\mathrm{~d} x}\right|_{\left|m_{j}\right|=\frac{3}{2}}\right) & =\frac{\alpha_{5 \mathrm{~S}}-\alpha_{0}}{\alpha_{5 \mathrm{~S}}-\alpha_{74 \mathrm{~S}}}
\end{aligned}
$$

The experimental slope is determined by linear fitting of the centers of the peaks. 1 The slopes in Fig. 7.6 are 2.43 for the level with $\left|m_{j}\right|=\frac{1}{2}$ and 1.32 for the level

\footnotetext{
${ }^{1}$ We set the y-intercept to zero for the linear fitting of the $\left|m_{j}\right|=\frac{3}{2}$ level, because this level is passing through the origin. For the case of $\left|m_{j}\right|=\frac{1}{2}$, we determine both the slope and the intercept by the linear fitting, because the $\left|m_{j}\right|=\frac{1}{2}$ level does have a small, negative intercept. This is because in the weak field regime, this level maps to $\left|F=3, m_{F}=0\right\rangle$, and thus $\alpha_{5 \mathrm{P}}=\alpha_{0}-\frac{4}{5} \alpha_{2}$ (in weak fields). If we use $\alpha_{5 \mathrm{P}}=\alpha_{0}-\alpha_{2}$ of the strong field regime and linearly fit this level, we will obtain a line with $\approx-30 \mathrm{MHz}$ y-intercept in our calculation when the lattice intensity maximum is $1.8 \times 10^{11} \mathrm{~W} / \mathrm{m}^{2}$.
} 
with $\left|m_{j}\right|=\frac{3}{2}$. Since the $\alpha_{5 S}$ and $\alpha_{74 S}$ are both well-known, from the two slopes, we can immediately calculate the value of $\alpha_{0}$ and $\alpha_{2}$. With $\alpha_{5 S}=687.3 \times 4 \pi \epsilon_{0}$ and $\alpha_{74 S}=-272.5 \times 4 \pi \epsilon_{0} a_{0}^{3}$, we calculate $\alpha_{0}=-1112.1 \times 4 \pi \epsilon_{0} a_{0}^{3}$ and $\alpha_{2}=534.9 \times 4 \pi \epsilon_{0} a_{0}^{3}$, respectively. Table. 7.2 shows the summary of the calculation.

The uncertainty listed in Table 7.2 is from error of the linear fitting, which is less than $1 \%$. The other sources of uncertainty are the calibration of the $\Delta$ blue and $\Delta$ red, both are less than $2 \%$. Overall, the uncertainty is small, and the values of $\alpha_{0}$ and $\alpha_{0}$ are reasonably close to the recent values from theoretical calculation.

The above analysis is our first experimental determination of $\alpha_{0}$ and $\alpha_{0}$, which demonstrates the method of determining the experimental value of $\alpha_{0}$ and $\alpha_{0}$ with two-photon spectroscopy of magic Rydberg states. To our best knowledge, there is no such experimental determination of the $\alpha_{0}$ and $\alpha_{0}$ of rubidium $5 \mathrm{P}_{3 / 2}$ in $1064 \mathrm{~nm}$ lattice light field in the literature.

Table 7.2: Work sheet of Fig. 7.6

\begin{tabular}{|c|c|c|c|}
\hline & value & error & reference \\
\hline \multicolumn{4}{|l|}{ waterfall plot } \\
\hline slope, $\left|m_{j}\right|=\frac{3}{2}$ & 1.32 & $2.75 \times 10^{-3}$ & \\
\hline slope, $\left|m_{j}\right|=\frac{1}{2}$ & 2.43 & $1.65 \times 10^{-2}$ & \\
\hline differential & 1.11 & $1.67 \times 10^{-2}$ & \\
\hline average & 1.87 & $8.34 \times 10^{-3}$ & \\
\hline \multicolumn{4}{|c|}{ dynamic polarizabilities from theoretical calculations in atomic unit } \\
\hline$\alpha_{5 \mathrm{~S}}$ & 687.3 & 0.5 & Table III of [40] \\
\hline$\alpha_{74 \mathrm{~S}}$ & -272.5 & 0.5 & [19]. The error is a conservative estimate. \\
\hline \multicolumn{4}{|c|}{ Experimental dynamic polarizabilities in atomic unit } \\
\hline$\alpha_{0}$ & -1112.1 & 8.1 & theoretical -1120 , Fig.2 of [40] \\
\hline$\alpha_{2}$ & 534.9 & 4.0 & theoretical 555, Fig.2 of [40] \\
\hline
\end{tabular}




\section{CHAPTER VIII}

\section{Spectroscopy of hydrogenic Rydberg states}

My experiment was originally designed for the study of the hydrogenic Rydberg states in $1064 \mathrm{~nm}$ optical lattices, which remains one of the long-term goals of the project. In this chapter, I give a brief review of the theoretical and experimental work done by the group and summarize the experimental progress made to date.

\subsection{Ponderomotive optical lattices}

The ponderomotive optical lattice $(\overline{\mathrm{POL}})$ trap for Rydberg atoms was proposed in 2000 by the group [19]. The experimantal demonstration of POL in the group can be found in references [20, 21]. Similar to conventional optical-lattice traps for groundstate atoms, POL utilizes the periodic potentials of a standing wave produced by counter-propagating laser beams to trap Rydberg atoms. The name "ponderomotive" comes from the pondermotive force experienced by the quasi-free Rydberg electron inside the rapidly oscillating light field. The quasi-free Rydberg electron is trapped by the ponderomotive force in lattice intensity minima. The entire Rydberg atom is then trapped in intensity minima because of the weak Coulomb binding between the atom core and the Rydberg electron.

There are two major differences between ponderomotive optical lattices and conventional optical lattices. First, Rydberg atoms are thousands of times larger than 


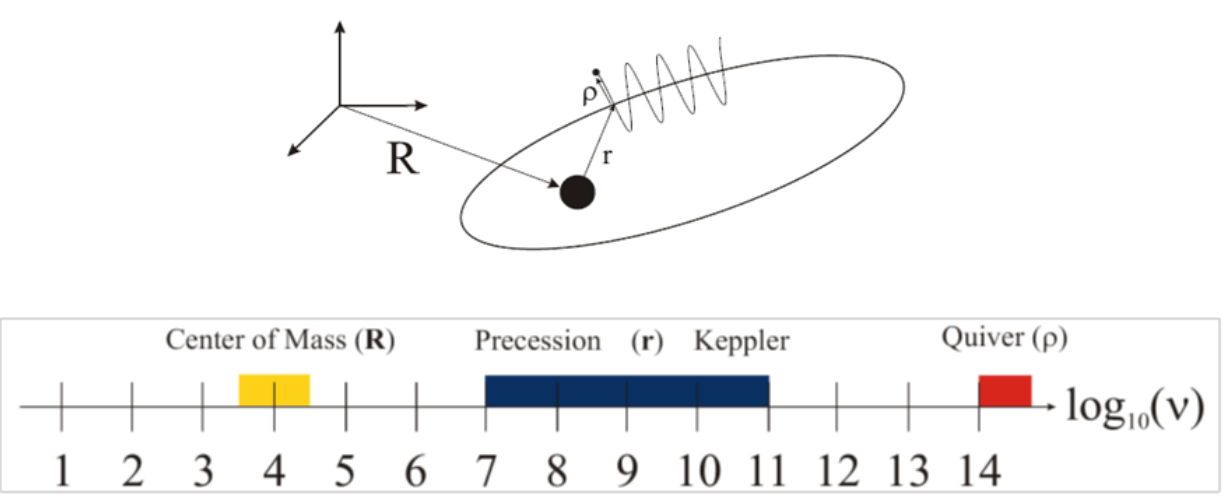

Figure 8.1:

Coordinates and time scales of a Rydberg atom in an optical lattice. (Figure adapted from reference [49].)

ground-state atoms. Ground-state atoms are approximately point-like inside an optical lattice. In contrast, the lattice potential seen by a Rydberg atom is the average of the free-electron ponderomotive potential over the large spatial extent of the Rydberg wave function, which is comparable to the dimension of the lattice period. Second, the number of the degenerate high angular momentum states in Rydberg atoms is large, and the adiabatic potentials of a Rydberg atom inside an optical lattice become mixed as the principal quantum number $n$ increases.

The motion of a Rydberg atom inside an optical lattice can be described by three coordinates: the center of mass coordinate $\mathbf{R}$, the position of the Rydberg electron relative to the core $\mathbf{r}$, and the quiver motion of the Rydberg electron $\boldsymbol{\rho}$, as shown in Fig. 8.1. The fastest motion is the quiver motion of the Rydberg electron, the relative motion is the second fastest, and the center of mass is the slowest. The times scales of each motion differs from others by a factor of about 1000. Under this condition, the adiabatic trapping potential experienced by the Rydberg atom inside a optical lattice can be calculated by applying the Born-Oppenheimer approximation. The adiabatic trapping potential resulting from the Born-Oppenheimer approximation is a spacial average of free-electron ponderomotive energy weighted by the Rydberg electron wave 
function,

$$
V_{\mathrm{ad}}(\mathbf{R})=\int d^{3} r V_{\mathrm{P}}(\mathbf{R}+\mathbf{r})|\psi(\mathbf{r})|^{2}
$$

where $V_{\mathrm{P}}$ is the free-electron ponderomotive energy,

$$
V_{\mathrm{P}}=\frac{e^{2} E_{0}^{2}}{4 m_{e} \omega^{2}}
$$

$E_{0}$ is the single-beam field amplitude, and $\omega$ is the angular frequency of the field.

Fig. 8.2 shows a plot of the adiabatic potentials and calculated two-photon excitation spectra, with full free-electron POL shift of $1 \mathrm{GHz}$. The binding energy is plotted in wavenumbers, 1 wavenumber in $\mathrm{cm}^{-1}=30 \mathrm{GHz}$. The following is a discussion of the plot.

(a) Quantum defect. The plot shows all Rydberg states near the principal quantum number $\mathrm{n}=66$, and we refer $66 \mathrm{H}$ to as the "hydrogenic" manifold, which includes the states with high angular momentum quantum number and negligible quantum defect. The binding energy of a Rydberg state is [5],

$$
E_{n l}=\frac{-\mathrm{Ryd}}{[n *]^{2}}=\frac{-\mathrm{Ryd}}{\left[n-\delta_{l}\right]^{2}}
$$

where Ryd is the Rydberg constant in wavenumber: $n *=n-\delta_{l}$ is the effective principal quantum number and $\delta$ is the quantum defect. For rubidium Rydberg atoms, $\delta_{s}=3.13, \delta_{p}=2.65, \delta_{d}=1.34, \delta_{f}=0.02$, and $\delta_{l} \simeq 0.00$, for $l>3$. Because of the value of the quantum defect, the energy level appears in the following order: $(\mathrm{n}+2) \mathrm{P},(\mathrm{n}+1) \mathrm{D},(\mathrm{n}+3) \mathrm{S}, \mathrm{nF}, \mathrm{nH}($ top-down).

(b) "Spaghetti plot", zoom-in of 66 H. Due to the Rydberg electron wavefunction averaging over the lattice potential wells, the Rydberg atom in a deep $1064 \mathrm{~nm}$ lattice sees a light shift that is not linearly related to the sinusoidal standing-

\footnotetext{
${ }^{1} \mathrm{R}_{\infty}=10973731.568539(55) \mathrm{m}^{-1}$, CODATA
} 


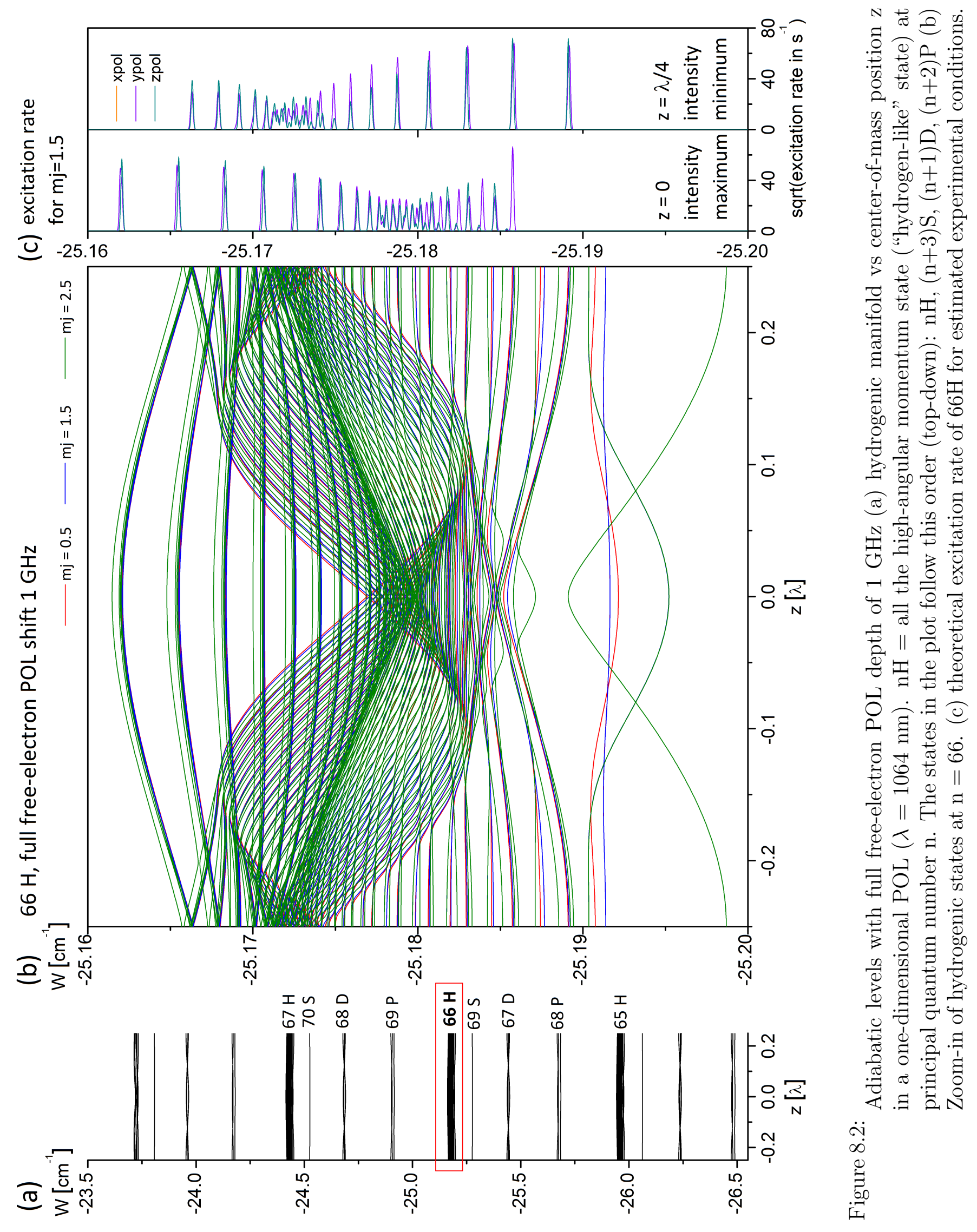


wave pattern of the lattice intensity modulation. In the case of $66 \mathrm{H}$, as shown in the plot (b), the size of the Rydberg atom is about equal to the lattice potential well periodicity. The spectral features of $66 \mathrm{H}$ hydrogenic states are the several strong rotor-state-like spectral lines around the lattice intensity maximum $(z=0)$ and minimum $\left(z= \pm \frac{\lambda}{2}\right)$. These state mimic the rotor states, because their energy level separations are not evenly spaced but become larger and larger.

(c) Calculated excitation spectra. Due to the mixing with low angular momentum states ( $\mathrm{S}$ and $\mathrm{D}$ state), the large light shift allows us to selectively excite the atoms from $5 \mathrm{P}_{3 / 2}$ intermediate state to the lattice-mixed states in (b). Electricdipole selection rule do not apply, because the lattice-mixed states all carry significant S and D character. An immediate experimental application would be to use the large light shift to prepare high angular momentum states. A typical preparation sequence would be: (1) Perform a two-photon excitation in the presence of $1064 \mathrm{~nm}$ light field and excite the atoms to the rotor states in Fig. 8.2(b). (2) Switch the lattice light off (one might consider both adiabatic of diabatic cases). The atoms excited to the lattice-mixed hydrogenic states will evolve into field-free high-angular-momentum hydrogenic states.

\subsection{Experimental progress}

The high $1064 \mathrm{~nm}$ laser intensity required for the spectroscopic study of hydrogenic Rydberg states is achieved by the near-concentric cavity setup. The cavity-generated deep lattice is loaded with atoms by lattice adiabatic compression, which is discussed in Chapter $\mathrm{V}$. The general spectroscopic procedure for the lattice-shifted hydrogenic states has two steps: First, calibrate the lower transition laser such that it is onresonant to the lattice-shifted $5 \mathrm{~S}_{1 / 2} \rightarrow 5 \mathrm{P}_{3 / 2}$ transition. Details of the calibration procedure is given in Appendix A. Second, scan the upper transition laser from the nearest lattice-shifted Rydberg $(\mathrm{n}+3) \mathrm{S}$ lines to the region where the lattice-shifted 
nH hydrogenic state should be. Fig. 8.3 shows an example of the relative spectral-line positions. The location of the lattice-shifted $66 \mathrm{~F}$ and $66 \mathrm{H}$ is about $2.4 \mathrm{GHz}$ above the lattice-shifted 69S line, and the hydrogenic manifolds spread over a spectral range of about $1 \mathrm{GHz}$. The spectroscopic study of hydrogenic states in $1064 \mathrm{~nm}$ lattice can be done with both ${ }^{87} \mathrm{Rb}$ and ${ }^{85} \mathrm{Rb}$ isotopes.

Fig. 8.4 shows a spectroscopic scan of a ${ }^{85} \mathrm{Rb}$ lattice-compressed sample in the region where the lattice-mixed hydrogenic states should be. The spectrum shows signals that occur at the location where the lattice-shifted $66 \mathrm{H}$ hydrogenic states should be. In this scan, the lower transition detuning is locked to $2220 \mathrm{MHz}$, which drives the transition from the upper $5 \mathrm{~S}_{1 / 2}$ hyperfine level to the intermediate state $5 \mathrm{P}_{3 / 2},\left|m_{j}\right|=\frac{1}{2}$. I scan the upper transition laser frequency with the step motor over the target region to get this spectrum. The experimental repetition rate is $100 \mathrm{~Hz}$, and each point on the spectrum is an average of 50 shots. The lattice power is increased from $2 \mathrm{~mW}$ to $25 \mathrm{~mW}$ in the experimental cycle, and the compression duration is $30 \mu$ s. I focus a pair of counter-propogating lower transition laser beams along the lattice axis, in order to balance the radiation pressure caused by the lower transition laser, whose intensity is about $100 I_{\text {sat }}$. The upper transition laser is also focused at

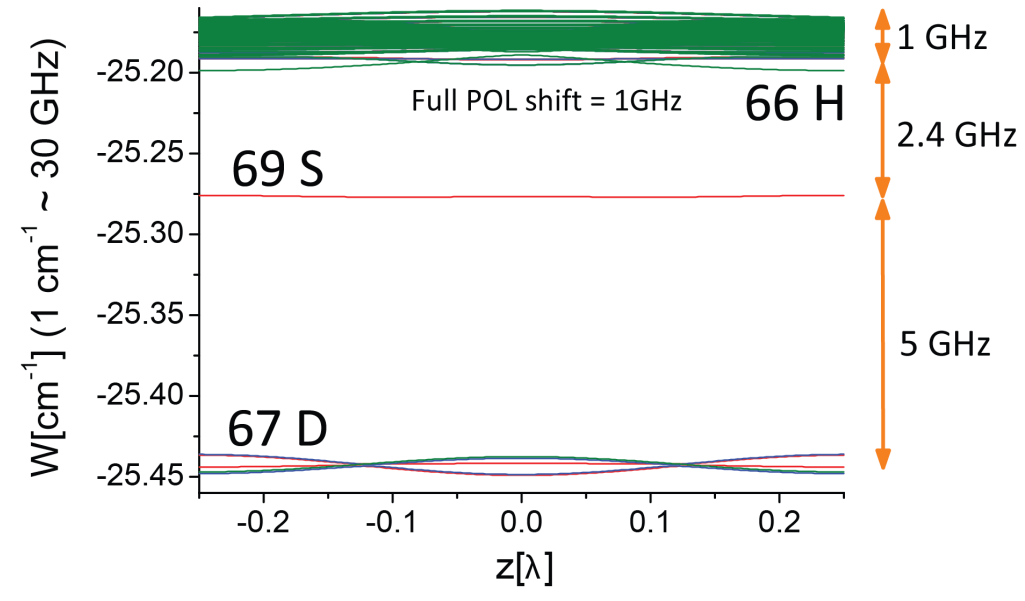

Figure 8.3: Relative spectral-line positions near the $66 \mathrm{H}$ in GHz, with full POL shift of $1 \mathrm{GHz}$. 
the trap center, but enters at an angle of $55^{\circ}$ relative to the lattice axis. The intensity of the upper transition laser at the focus is about $6 \times 10^{6} \mathrm{~mW} / \mathrm{cm}^{2}$. Both Rydberg excitation lasers are pulse on for $40 \mu \mathrm{s}$ when the lattice is held at high power. The field-ionization ramp is on after the excitation, and the lattice returns to the loading power $14 \mu$ s after the ionization field is on.

At $0 \mathrm{MHz}$ on the spectra, there is an off-resonantly excited $69 \mathrm{~S}$ MOT-atom signal from the lower $5 \mathrm{~S}_{1 / 2}$ hyperfine level. This level is about $3 \mathrm{GHz}$ below the upper $5 \mathrm{~S}_{1 / 2}$ hyperfine level, so it appears at the low frequency side of the target region of the lattice-shifted hydrogenic states; I use this $69 \mathrm{~S}$ lower level line as a convenient frequency marker. The signal-to-noise ratio of the lattice-shifted signals is low, so I cannot conclude that the signal is indeed from the hydrogenic state mixed by the $1064 \mathrm{~nm}$ lattice. Also, there is no clear "wiggle" pattern on the spectrum, which would match the calculated spectral features of the rotor states in Fig. 8.2.

The low signal-to noise-ratio is not unexpected. The count rate of the latticemixed hydrogenic states is very low - for the case of $66 \mathrm{H}$ in a $1 \mathrm{GHz}$ deep POL, the count rate of $66 \mathrm{~F}$ lines is calculated to be about $5 \%$ of the nearest lattice-shifted 69S line, and the strength of rotor states of the hydrogenic manifolds is less than

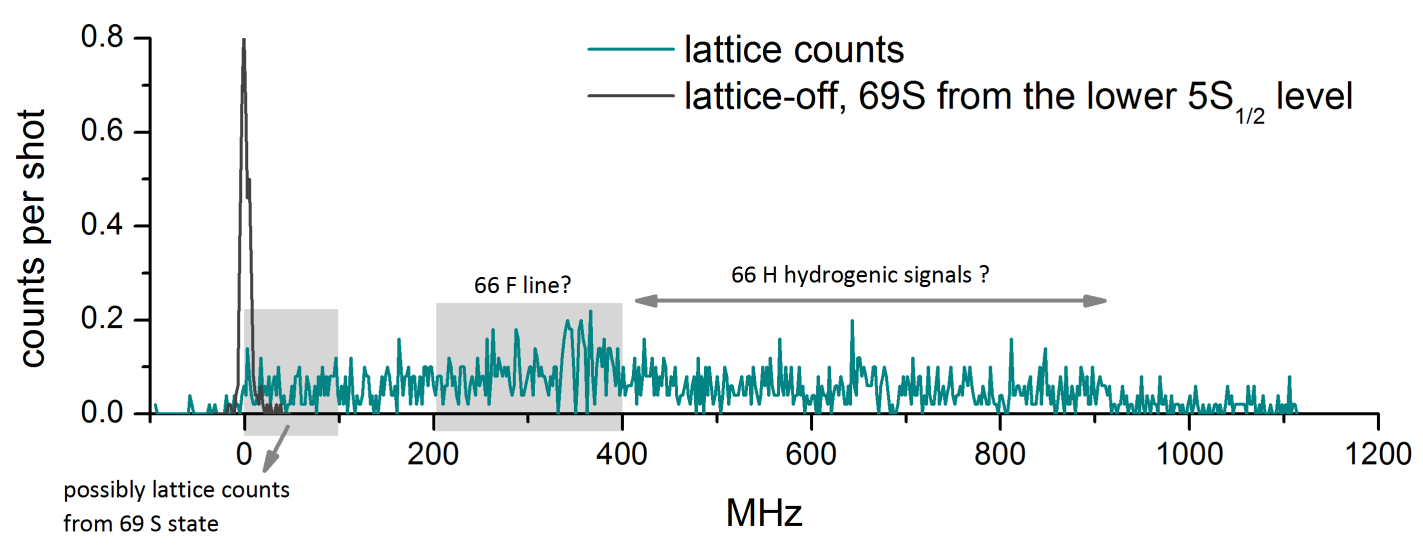

Figure 8.4: Experimental scan that shows possible, but not certain, signal from the lattice-shifted hydrogenic states. 
$5 \%$. In future attempts to search for the hydrogenic states in a $1064 \mathrm{~nm}$ lattice, we should try to use a higher lattice power to acquire stronger lattice light shift; in that case we will get a stronger mixing of low angular momentum into the hydrogenic manifold, and thus a higher count rate. Also, we should use the highest sub-level of $5 \mathrm{P}_{3 / 2},\left|m_{j}\right|=\frac{3}{2}$ as the intermediate launch state. As Fig. 7.5 shows, the two-photon Rydberg excitation through this intermediate state results in a narrower linewidth and a higher count rate than excitation from the highest level of $5 \mathrm{P}_{3 / 2},\left|m_{j}\right|=\frac{1}{2}$, which is what I used for taking the spectrum in Fig. 8.4. 


\section{CHAPTER IX}

\section{Summary and future works}

At the point when I took over this work, we had most of the vacuum components machined or purchased. I continued testing and improving the designing of the cavity components, and I finalized the cavity fabrication so that it became ready for operation in a vacuum environment. In the meantime, I built the entire laser-cooling experimental apparatus for this cavity experiment. My contribution to the group is realizing this near-concentric cavity experimental setup and performing an initial set of experiments with the cavity-generated optical lattice. They have been described in detail in this thesis. My thesis also includes several chapters on the technical realization of the near-concentric cavity lattice; those chapters will serve as a reference for future graduate students.

In addition to cavity QED [50] experiments utilizing high-finesse cavities, there are many recent efforts exploring various applications of in-vaccum cavities, such as cavity-enhanced cold-atom memory [51], low-finesse cavities for atom interferometry [52], or multimode cavities for BEC experiments [53]. A near-concentric cavity has also been used for single-atom detection [54]. As far as we know, our near-concentric cavity is a unique experimental tool for optical-lattice experiments, in which an extremely deep optical lattice adiabatically compresses the atom sample and generates enormous light shifts. 
As a summary of this thesis, here are several ideas for the future work.

- In the near future, we will continue to spectroscopically explore the hydrogenic Rydberg states.

- The spectroscopy of Rydberg molecules [55, 56] is another experiment that could possibly be demonstrated in the near future. The high atomic volume density in lattice potential wells with periodicity of $532 \mathrm{~nm}$, along with the fast experimental repetition rate, would solve some difficulties experienced by previous Rydberg-molecule experiments in the group.

- We could also measure the vector polarizability $\alpha_{1}$ of $5 \mathrm{P}_{3 / 2}$ [40] in a circularlypolarized lattice light field.

- The parallel, elongated cold-atom channels in the Hermite-Gaussian cavity modes offer a platform for experiments using Rydberg blockade [11 13], Rydberg EIT [43, 44], and Rydberg polaritons [45]. The Rydberg EIT could possibly be demonstrated in the near future, since our group already has sufficient experience with Rydberg EIT in a room-temperature vapor cell. 
APPENDIX 


\section{APPENDIX A}

\section{Lower-transition laser frequency calibration}

To excite the ground-state atoms to the Rydberg state in the presence of lattice light field, the lower-transition laser frequency is locked so that the laser on-resonantly drives a component of the lattice-shifted $5 \mathrm{~S}_{1 / 2} \rightarrow 5 \mathrm{P}_{3 / 2}$ transition, which is the lower transition. The lattice-induced light shift by a deep optical lattice is usually several GHz. The calibration of the lower transition detuning relative to the lattice power is done by the spectroscopy of the Rydberg S line. In this Appendix, I use the trapped atoms from the $5 \mathrm{P}_{3 / 2},\left|m_{j}\right|=\frac{1}{2}$ intermediate state as an example. Fig. A.1 shows the spectroscopic scans for the calibration.

The on-resonance detuning is the frequency-setting where there is only one strong spectral line on the spectrum, with a weak tail on the higher frequency side, as in the scan in Fig. A.1(b). Notice that the strong peak slightly splits, because the uppermost spectral line of from the $5 \mathrm{P}_{3 / 2},\left|m_{j}\right|=\frac{1}{2}$ intermediate state is composed of two levels with very close energy splitting. For the cases in Fig. A.1(c)(e)(f)(i), the lower transition detuning is not high enough for the corresponding lattice power, so that there is a smaller peak on the higher frequency side of the strong line; the smaller

peak are from the lower sub-levels of $5 \mathrm{P}_{3 / 2},\left|m_{j}\right|=\frac{1}{2}$ in Fig. 7.4. For the cases in Fig. A.1 (a) (d) (g) (h), the lower transition frequency is too high, so there are not many 

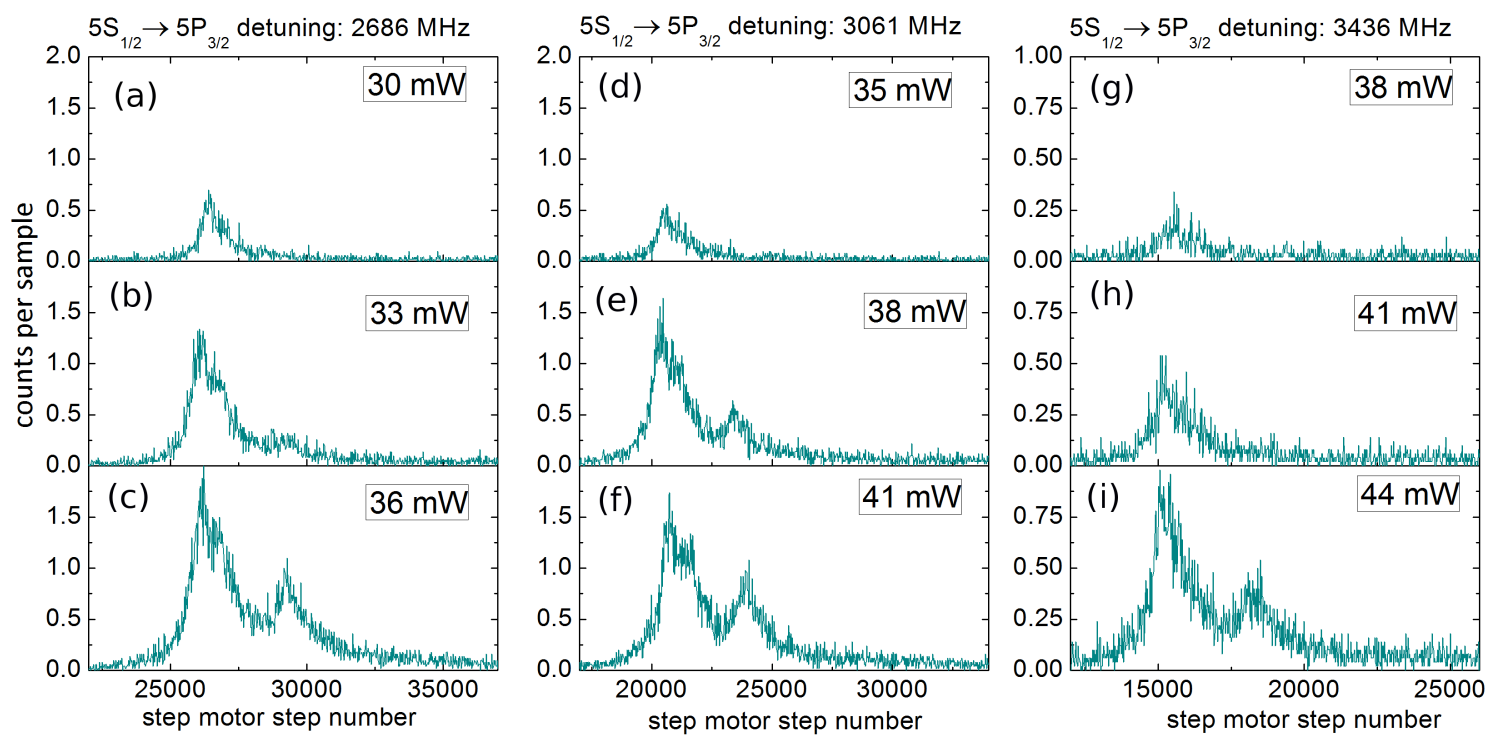

Figure A.1:

$69 \mathrm{~S}$ spectra for ${ }^{87} \mathrm{Rb}$. The atoms are from the $5 \mathrm{P}_{3 / 2},\left|m_{j}\right|=\frac{1}{2}$ intermediate state. The lower transition laser co-propagates with the lattice laser, and is focused into the cavity with an intensity about $50 I_{\text {sat }}$.

atoms populating in the uppermost sub-levels of the $5 \mathrm{P}_{3 / 2},\left|m_{j}\right|=\frac{1}{2}$ intermediate state. There is only one spectral peak without substructure, and the count rate of Rydberg S line is about half of the on-resonance signal, as in Fig. A.1(b).

From the best case, which is Fig. A.1(b), the lower transition calibration factor for the topmost $5 \mathrm{P}_{3 / 2},\left|m_{j}\right|=\frac{1}{2}$ level is calculated as

$\frac{5 \mathrm{~S}_{1 / 2} \rightarrow 5 \mathrm{P}_{3 / 2} \text { detuning in } \mathrm{MHz}}{\text { lattice power in } \mathrm{mW}}=\frac{2686}{33}=81 \mathrm{MHz}$ per $\mathrm{mW}$ of lattice power (A.1) 


\section{BIBLIOGRAPHY}




\section{BIBLIOGRAPHY}

[1] Daryl W. Preston. Doppler-free saturated absorption: Laser spectroscopy. Am. J. Phys., 64, 1996.

[2] A. E. Siegman. Lasers. University Science Books, Sausalito, CA, 1986.

[3] Daniel A. Steck. Rubidium 87 d line data. http://steck.us/alkalidata, .

[4] Daniel A. Steck. Rubidium 85 d line data. http://steck.us/alkalidata, .

[5] T. F. Gallagher. Rydberg Atoms. Cambridge University Press, Cambridge, 1994.

[6] Jonathon A. Sedlacek, Arne Schwettmann, Harald Kübler, Robert Löw, Tilman Pfau, and James P. Shaffer. Microwave electrometry with rydberg atoms in a vapour cell using bright atomic resonances. Nature Physics, 8:819-824, 2012.

[7] J. Liang, M. Gross, P. Goy, and S. Haroche. Circular rydberg-state spectroscopy. Phys. Rev. A, 33:4437, 1986.

[8] D. Jaksch, J. I. Cirac, P. Zoller, S. L. Rolston, R. Côté, and M. D. Lukin. Fast quantum gates for neutral atoms. Phys. Rev. Lett., 85:2208-2211, 2000.

[9] M. D. Lukin, M. Fleischhauer, R. Cote, L.-M. Duan, D. Jaksch, J. I. Cirac, and P. Zoller. Dipole blockade and quantum information processing in mesoscopic atomic ensembles. Phys. Rev. Lett., 87:037901, 2001.

[10] M. Saffman, T. G. Walker, and K. Mølmer. Quantum information with rydberg atoms. Rev. Mod. Phys., 82(2313), 2010.

[11] X. L. Zhang, L. Isenhower, A. T. Gill, T. G. Walker, and M. Saffman. Deterministic entanglement of two neutral atoms via rydberg blockade. Phys. Rev. A, 82(030306(R)), 2010.

[12] T. Wilk, A. Gaëtan, C. Evellin, J. Wolters, Y. Miroshnychenko, P. Grangier, and A. Browaeys. Entanglement of two individual neutral atoms using rydberg blockade. Phys. Rev. Lett., 104:010502, 2010.

[13] L. Isenhower, E. Urban, X. L. Zhang, A. T. Gill, T. Henage, T. A. Johnson, T. G. Walker, and M. Saffman. Demonstration of a neutral atom controlled-not quantum gate. Phys. Rev. Lett., 104:010503, 2010. 
[14] S. D. Hogan and F. Merkt. Demonstration of three-dimensional electrostatic trapping of state-selected rydberg atoms. Phys. Rev. Lett., 100:043001, 2008.

[15] J.-H. Choi, J. R. Guest, A. P. Povilus, E. Hansis, and G. Raithel. Magnetic trapping of long-lived cold rydberg atoms. Phys. Rev. Lett., 95:243001, 2005.

[16] P. H. Bucksbaum, R. R. Freeman, M. Bashkansky, and T. J. McIlrath. Role of the ponderomotive potential in above-threshold ionization. JOSA B, 4:760-764, 1987.

[17] P. H. Bucksbaum, D. W. Schumacher, and M. Bashkansky. High-intensity kapitza-dirac effect. Phys. Rev. Lett., 61:1182, 1988.

[18] R. R. Freeman, P. H. Bucksbaum, H. Milchberg, S. Darack, D. Schumacher, and M. E. Geusic. Above-threshold ionization with subpicosecond laser pulses. Phys. Rev. Lett., 59:1092, 1987.

[19] S. K. Dutta, J. R. Guest, D. Feldbaum, A. Walz-Flannigan, and G. Raithel. Ponderomotive optical lattice for rydberg atoms. Phys. Rev. Lett., 85:5551, 2000.

[20] S. E. Anderson, K. C.Younge, and G. Raithel. Trapping rydberg atoms in an optical lattice. Phys. Rev. Lett., 107:263001, 2011.

[21] K. C.Younge, B. Knuffman, S. E. Anderson, and G. Raithel. State-dependent energy shifts of rydberg atoms in a ponderomotive optical lattices. Phys. Rev. Lett., 104:173001, 2010.

[22] S. E. Anderson and G. Raithel. Dependence of rydberg-atom optical lattices on the angular wave function. Phys. Rev. Lett., 109:023001, 2012.

[23] Sarah E. Anderson and Georg Raithel. Ionization of rydberg atoms by standingwave light fields. Nature Communications, 4, 2013.

[24] Kaitlin R. Moore, Sarah E. Anderson, and Georg Raithel. Forbidden atomic transitions driven by an intensity-modulated laser trap. Nature Communications, 6(6090), 2015.

[25] Kelly Cooper Younge, Sarah Elizabeth Anderson, and Georg Raithel. Adiabatic potentials for rydberg atoms in a ponderomotive optical lattice. New J. Phys., 12:023031, 2010.

[26] M. A. Bandres and J. C. Gutiérrez-Vega. Ince-gaussian beams. Optics Letters, 29:144-146, 2004.

[27] Ulrich T. Schwarz, Miguel A. Bandres, and Julio C. Gutiérrez-Vega. Observation of ince-gaussian modes in stable resonators. Optics Letters, 29(16):1870-1872, 2004.

[28] E. D. Black. An introduction to pound--drever-hall laser frequency stabilization. Am. J. Phys., 69:79-87, 2001. 
[29] A. S. Arnold, J. S. Wilson, and M. G. Boshier. A simple extended-cavity diode laser. Rev. Sci. Instrum., 69:1236, 1998.

[30] E. Hansis, T. Cubel, J.-H. Choi, J. R. Guest, and G. Raithel. Simple pressuretuned fabry-pérot interferometer. Rev. Sci. Instrum., 76:033105, 2005.

[31] Wolfgang Demtröder. Laser spectroscopy: Vol. 2 experimental techniques. Springer-Verlag, 2008.

[32] Christopher J.Foot. Atomic Physics. Oxford University Press, 2010.

[33] Joseph L.Wiza. Microchannel plate detectors. Nuclear Instruments and Methods, 162:587-601, 1979.

[34] H. J. Metcalf and P. van der Straten. Laser Cooling and Trapping. Springer, Berlin, 1999.

[35] A. G. Truscott, M. E. J. Friese, N. R. Heckenberg, and H. Rubinsztein-Dunlop. Optically written waveguide in an atomic vapor. Phys. Rev. Lett., 82:1438, 1999.

[36] Mukund Vengalattore and Mara Prentiss. Radial confinement of light in an ultracold anisotropic medium. Phys. Rev. Lett., 95:243601, 2005.

[37] P. R. Berman and V. S. Malinovsky. Principles of Laser Spectroscopy and Quantum Optics. Princeton University Press, Princeton, NJ, 2011.

[38] William Guerin, Franck Michaud, and Robin Kaiser. Mechanisms for lasing with cold atoms as the gain medium. Phys. Rev. Lett., 101:093002, 2008.

[39] Geert Vrijsen, Onur Hosten, Jongmin Lee, Simon Bernon, and Mark A. Kasevich. Raman lasing with a cold atom gain medium in a high-finesse optical cavity. Phys. Rev. Lett., 107:063904, 2011.

[40] Bindiya Arora and B. K. Sahoo. State-insensitive trapping of rb atoms: Linearly versus circularly polarized light. Phys. Rev. A, 86:033416, 2012.

[41] Zheng-Min Wang and D. S. Elliott. Determination of cross sections and continuum phases of rubidium through complete measurements of atomic multiphoton ionization. Phys. Rev. Lett., 84(17):3795-3798, 2000.

[42] B. Knuffman, A. V. Steele, J. Orloff, and J. J. McClelland. Nanoscale focused ion beam from laser-cooled lithium atoms. New J. Phys., 13:103035, 2011.

[43] Thibault Peyronel, Ofer Firstenberg, Qi-Yu Liang, Sebastian Hofferberth, Alexey V. Gorshkov, Thomas Pohl, Mikhail D. Lukin, and Vladan Vuletić. Quantum nonlinear optics with single photons enabled by strongly interacting atoms. Nature, 488:57-60, 2012. 
[44] David Petrosyan, Johannes Otterbach, and Michael Fleischhauer. Electromagnetically induced transparency with rydberg atoms. Phys. Rev. Lett., 107:213601, 2011.

[45] D. Maxwell, D. J. Szwer, D. Paredes-Barato, H. Busche, J. D. Pritchard, A. Gauguet, K. J. Weatherill, M. P. A. Jones, and C. S. Adams. Storage and control of optical photons using rydberg polaritons. Phys. Rev. Lett., 110(103001), 2013.

[46] M. Safronova, Bindiya Arora, and Charles W. Clark. Frequency-dependent polarizabilities of alkali-metal atoms from ultraviolet through infrared spectral regions. Phys. Rev. A, 73:022505, 2006.

[47] M. Marinescu, H. R. Sadeghpour, and A. Dalgarno. Dynamic dipole polarizabilities of rubidium. Phys. Rev. A, 49:5103, 1994.

[48] Keith D. Bonin and Michael A. Kadar-Kallen. Theory of the light-force technique for measuring polarizabilities. Phys. Rev. A, 47(944), 1993.

[49] Brenton J. Knuffman. Rydberg atoms in ponderomotive potentials. PhD thesis, The University of Michigan, 2009.

[50] Helmut Ritsch, Peter Domokos, Ferdinand Brennecke, and Tilman Esslinger. Cold atoms in cavity-generated dynamical optical potentials. Rev. Mod. Phys., 85:553-601, 2013.

[51] Erwan Bimbard, Rajiv Boddeda, Nicolas Vitrant, Andrey Grankin, Valentina Parigi, Jovica Stanojevic, Alexei Ourjoumtsev, and Philippe Grangier. Homodyne tomography of a single photon retrieved on demand from a cavity-enhanced cold atom memory. Phys. Rev. Lett., 112:033601, 2013.

[52] Paul Hamilton, Matt Jaffe, Justin M. Brown, Lothar Maisenbacher, Brian Estey, and Holger Müller. Atom interferometry in an optical cavity. Phys. Rev. Lett., 114:100405, 2015.

[53] Alicia J Kollár, Alexander T Papageorge, Kristian Baumann, Michael A Armen, and Benjamin L Lev. An adjustable-length cavity and bose-einstein condensate apparatus for multimode cavity qed. New J. Phys., 17:043012, 2015.

[54] A. Haase, B. Hessmo, and J. Schmiedmayer. Detecting magnetically guided atoms with an optical cavity. Optics Letters, 31:268-270, 2006.

[55] D. A. Anderson, S. A. Miller, and G. Raithel. Photoassociation of long-range nd rydberg molecules. Phys. Rev. Lett., 112:163201, 2014.

[56] Heiner Saßmannshausen, Frédéric Merkt, and Johannes Deiglmayr. Experimental characterization of singlet scattering channels in long-range rydberg molecules. Phys. Rev. Lett., 114:133201, 2015. 